\title{
THE RESPONSIBLE FASHION SYSTEM: A RE-CONCEPTUALIZATION OF THE ROLE OF FASHION DESIGN
}

\author{
by \\ Anika Kozlowski \\ B.Sc. (Hons) Science, University of Manitoba, 2003 \\ BDes. Fashion Design, Ryerson University, 2008 \\ MASc. Environmental Applied Science and Management, Ryerson University, 2012 \\ A dissertation presented to Ryerson University \\ in partial fulfillment of the \\ requirements for the degree of \\ Doctor of Philosophy \\ in the program of \\ Environmental Applied Science and Management
}

Toronto, Ontario, Canada 2019

(C) Anika Kozlowski, 2019 


\section{AUTHOR'S DECLARATION FOR ELECTRONIC SUBMISSION OF A DISSERTATION}

I hereby declare that I am the sole author of this dissertation. This is a true copy of the dissertation, including any final revisions, as accepted by my examiners.

I authorize Ryerson University to lend this dissertation to other institutions or individuals for the purpose of scholarly research.

I further authorize Ryerson University to reproduce this dissertation by photocopying or by other means, in total or in part, at the request of other institutions or individuals for the purpose of scholarly research.

I understand that my dissertation may be made electronically available to the public. 
The Responsible Fashion System: A Re-conceptualization of the Role of Fashion Design

\author{
Anika Kozlowski \\ $\mathrm{PhD}, 2019$ \\ Environmental Applied Science and Management \\ Ryerson University
}

\begin{abstract}
Sustainable fashion has developed as a response to the growing prominence and awareness of the negative environmental and social impacts of fashion apparel throughout its life cycle.

Responses to these wide-scale impacts have focused on piecemeal strategies that lack a cohesive perspective. The notion of design thinking and a holistic viewpoint are increasingly being seen as valued strategies for developing a sustainable fashion system. Fashion designers generally lack the tools to enable change and are caught within a system that cannot fulfill the potential of design-driven solutions for sustainability. Transformations to the design process, business practices, consumer behaviours and supply-chain sustainability are needed. This dissertation presents a series of manuscripts investigating a re-conceptualization of fashion design for system sustainability. Concepts put forth in the first manuscript, Theorizing the Fashion System provide context for a design focus. This study reviews existing theories of fashion production and consumption, for the purpose of establishing a theoretical framework to support subsequent research and tool design. The second manuscript Tools for Sustainable Fashion Design: An Analysis of their Fitness for Purpose examines existing design tools developed specifically for sustainable fashion designers. This research led to the creation and proposal of two conceptual frameworks: an innovation framework and five-dimensional model of sustainable fashion. Using the frameworks to analyze the tools and sustainable strategies within the tools resulted in the identification of three tool archetypes: 1) Universal, 2) Participatory and 3) Assessment. The third manuscript investigates and analyzes current design practices of sustainable fashion micro and small enterprises (MSE) and available sustainable design tools. The fourth manuscript, The reDesign Canvas: Fashion Design as a Tool for Sustainability, is a qualitative in-depth case study with a small fashion start-up. Utilizing observations in the field, interviews and design sessions, this study was able to identify leverage points within the design process to integrate sustainable strategies. The data collected informed the development of a sustainable fashion
\end{abstract}


design tool, the reDesign Canvas. This framework was tested and refined with the case study. This work aims to contribute a reconceptualization of the fashion design process to provide designers with the tools necessary to achieve a sustainable fashion system. 


\section{ACKNOWLEDGEMENTS}

Firstly, I would like to express my sincere gratitude to my advisors Dr. Michal Bardecki and Dr. Cory Searcy for their continuous support of my $\mathrm{Ph} . \mathrm{D}$. study and related research, for their patience, motivation, and immense knowledge. Throughout the writing of this dissertation I have received a great deal of assistance and I appreciate all of their contributions of time, ideas, and funding to make my Ph.D. experience productive and stimulating. A highly unusual pairing of a geographer, engineer and fashion designer has resulted in a rich, rewarding and fascinating journey into the world of sustainable fashion research. It is with immense gratitude that I thank my advisors for taking a chance on a fashion student interested in sustainability. The joy and enthusiasm they have for research was contagious and motivational for me, even during tough times in the Ph.D. pursuit. I could not have imagined having better advisors and mentors for my Ph.D. study. I am also thankful for the excellent example they have provided as successful researchers and professors.

I would like to thank my friends and colleagues at Ryerson, Kirsten, Joshua and Shelley. Thank you for all your help, jokes, support and friendship.

Last but not least, I owe a huge debt of gratitude to my family for all their love and encouragement. Nobody has been more important for me in the completion of this dissertation than my family members. I would like to thank my parents for all their love, help and support throughout this challenging work and time in my life. I wish to give thanks to my loving husband David for his patience, inspiration and immense understanding. I am so lucky and grateful for you, thank you for standing by my side. 


\section{Table of Contents}

Author's Declaration for Electronic Submission of a Dissertation ...............................................................ii

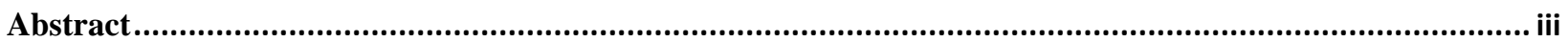

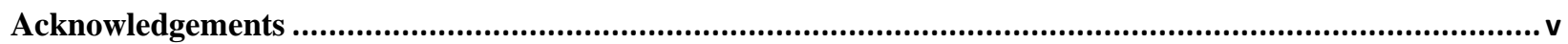

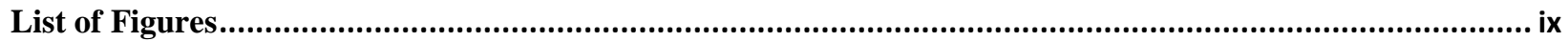

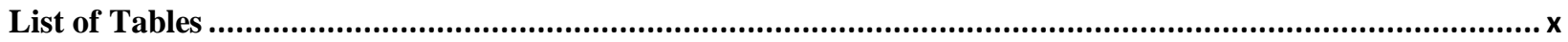

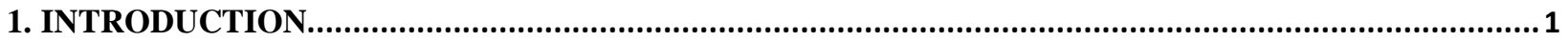

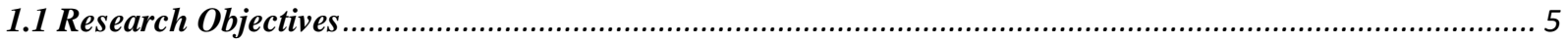

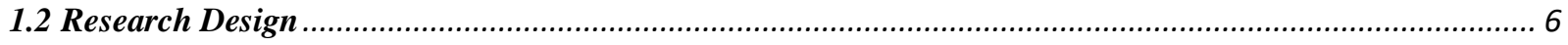

1.3 Outline of Chapters and Authorship Statement ……............................................................................. 7

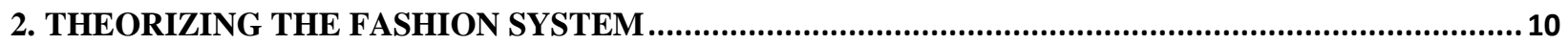

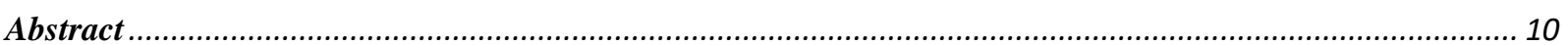

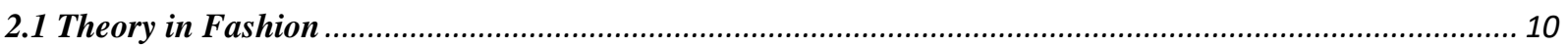

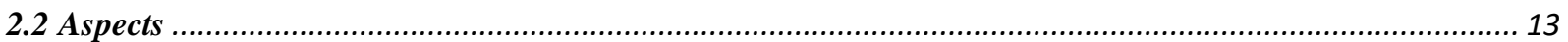

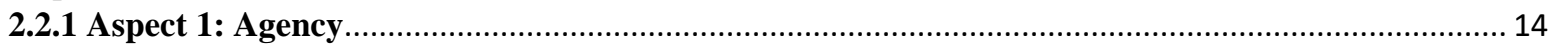

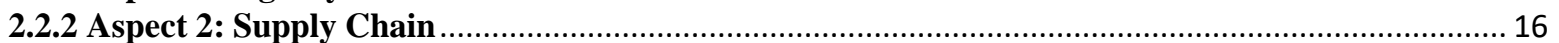

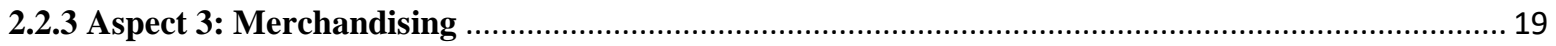

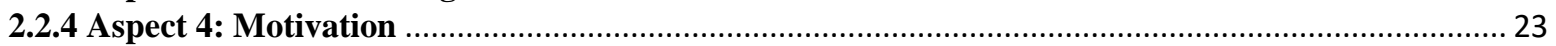

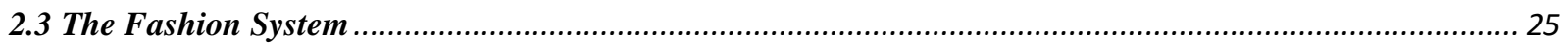

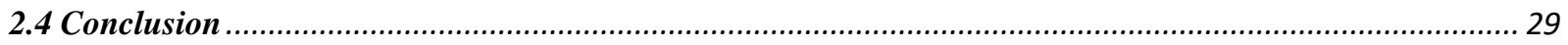

3. TOOLS FOR SUSTAINABLE FASHION DESIGN: AN ANALYSIS OF THEIR FITNESS FOR

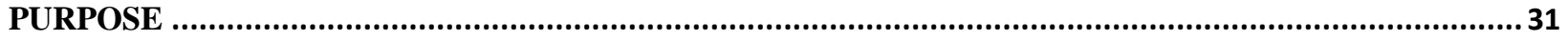

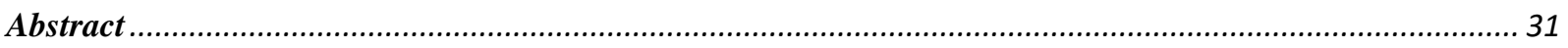

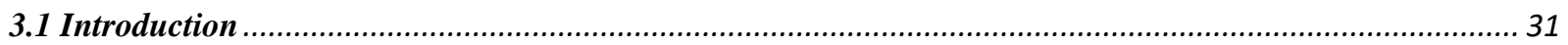

3.2 Analytical Framework

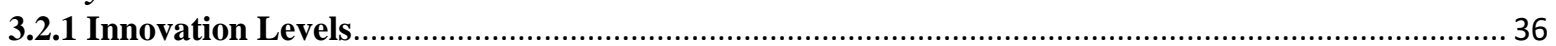

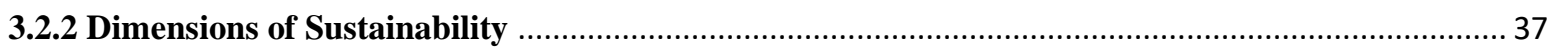

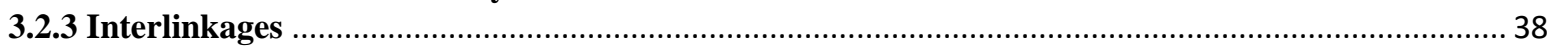

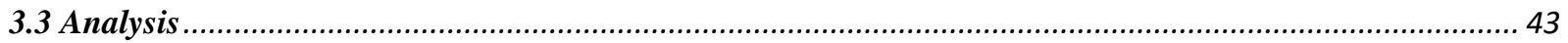

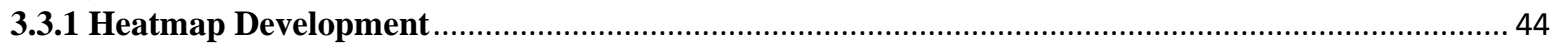

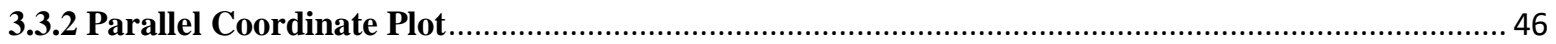

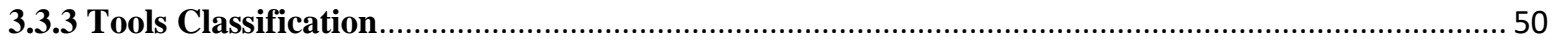

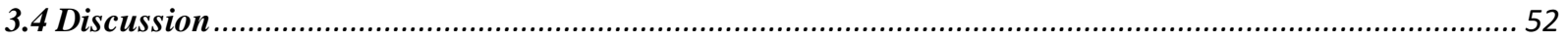

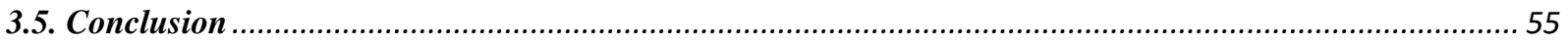

4. A CASE STUDY OF SUSTAINABLE FASHION MSE'S AND THEIR SUSTAINABLE DESIGN

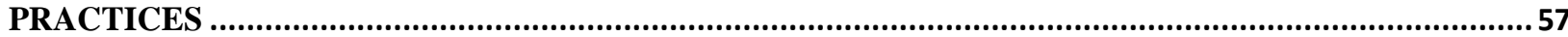


4.1 Introduction

5. THE reDESIGN CANVAS: FASHION DESIGN AS A TOOL FOR SUSTAINABILITY .......................93

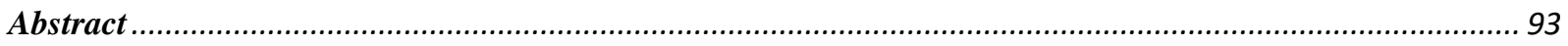

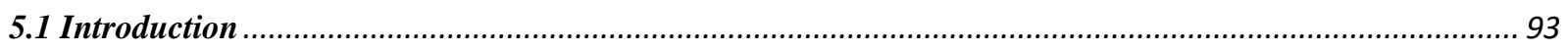

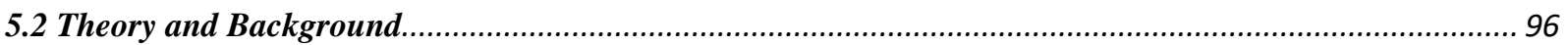

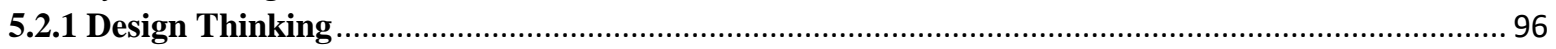

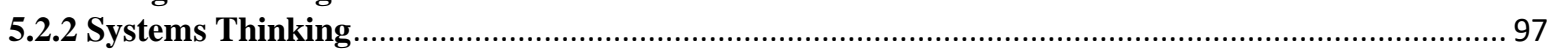

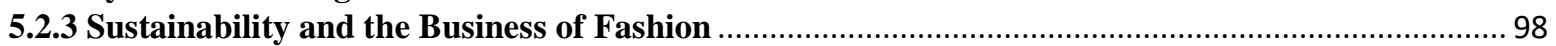

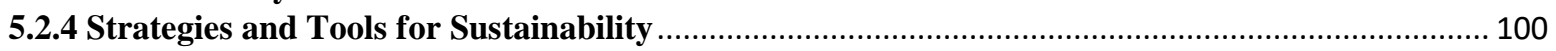

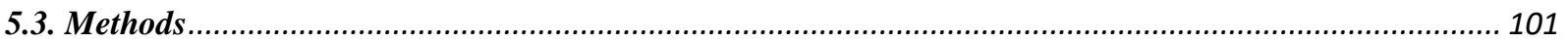

5.3.1 Conceptual Development of Initial reDesign Canvas................................................................. 102

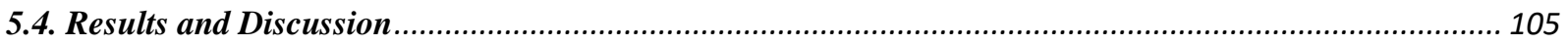

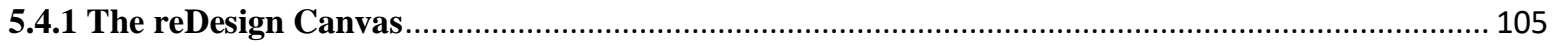

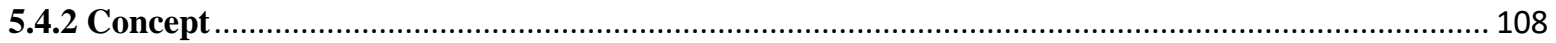

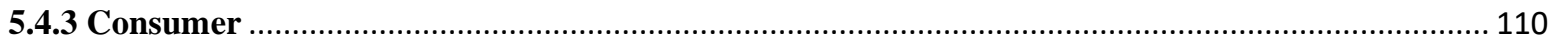

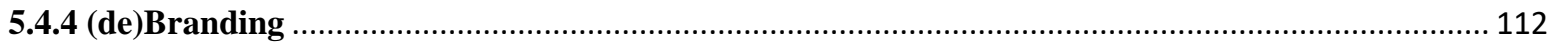

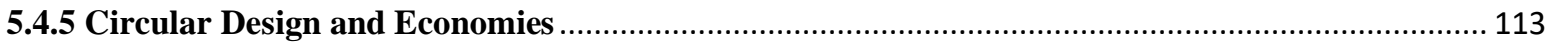

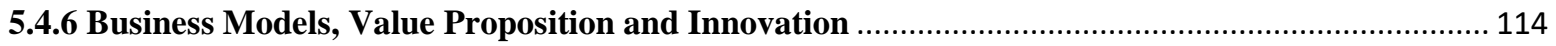

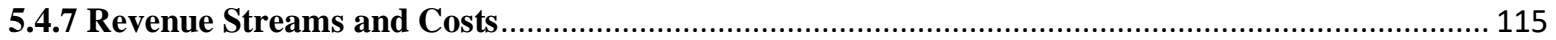

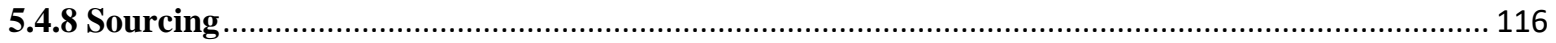

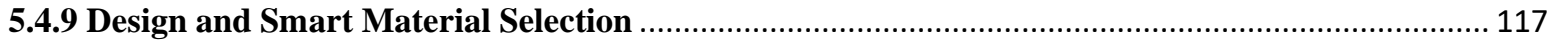

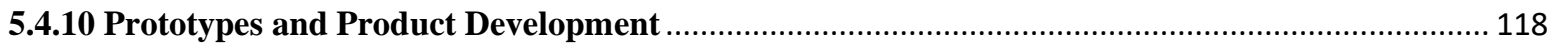

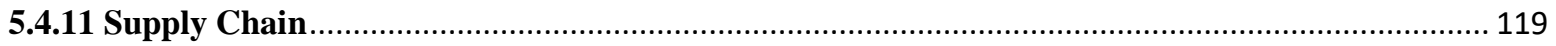

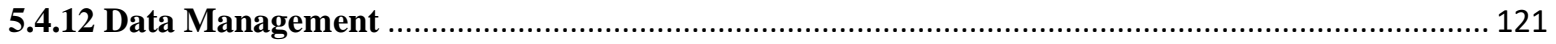

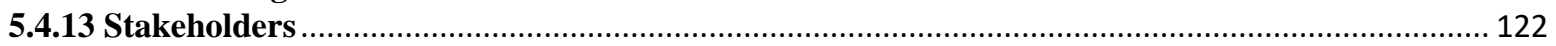

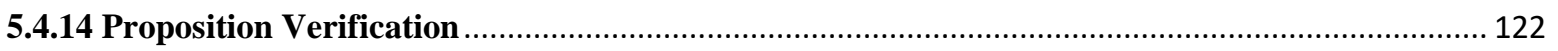

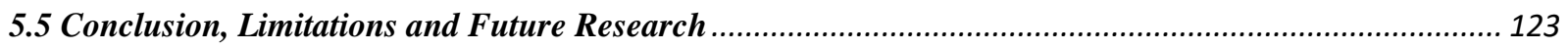

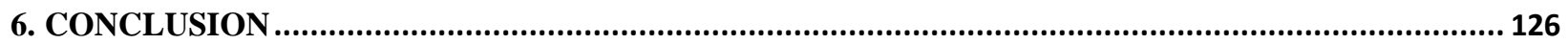

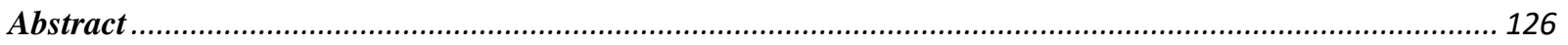

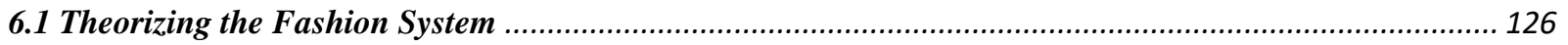

6.2 Tools for Sustainable Fashion Design: An Analysis of their Fitness for Purpose .................................. 127 
6.3 A Case Study of Sustainable Fashion MSEs and their Sustainable Design Practices

6.4 The reDesign Canvas: Fashion Design as a Tool for Sustainability ................................................ 129

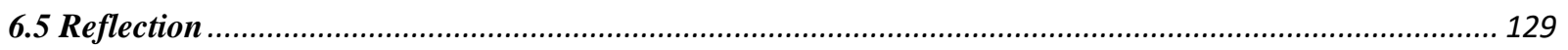

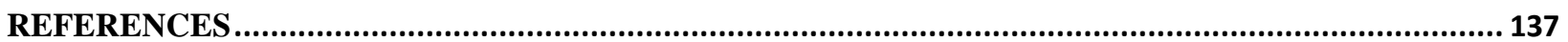




\section{List of Figures}

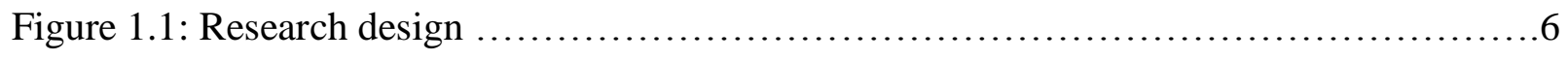

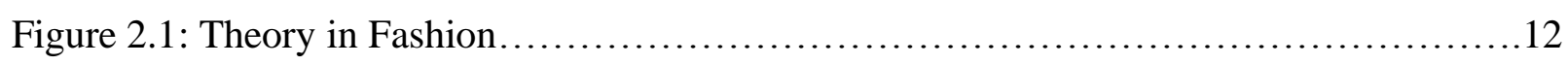

Figure 2.2: Agency in Fashion (following Bourdieu's Theory of Social Practice)..............15

Figure 2.3: Systems of Provision in Fashion...................................... 17

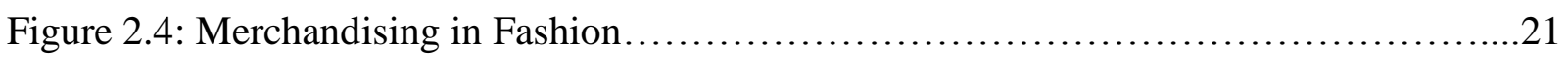

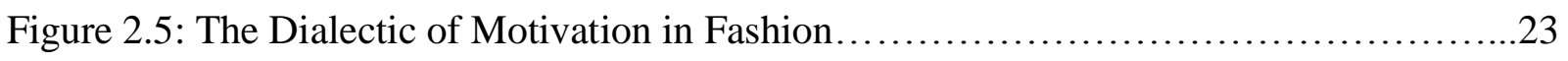

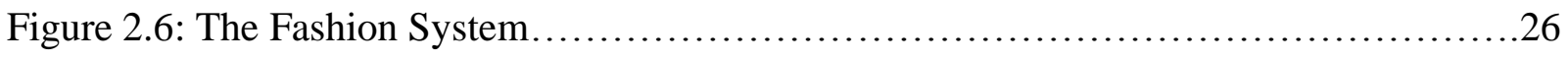

Figure 2.7: Examples of Areas of Research Interest in Fashion.........................28

Figure 3.1: Five dimensions of sustainable fashion..................................... 37

Figure 3.2: Heatmap of strategies for sustainable fashion design $\ldots \ldots \ldots \ldots \ldots \ldots \ldots \ldots \ldots \ldots . \ldots \ldots$

Figure 3.3: Parallel coordinate plot of the Tools..................................49

Figure 3.4: Classification of Tools........................................... 51

Figure 5.1: Research design framework....................................... 102

Figure 5.2: The iterative cycle of PAR (adapted from Walter, 2009)..........................

Figure 5.3: Initial draft of the reDesign canvas .................................. 106

Figure 5.4: Final version of the reDesign canvas................................. 107

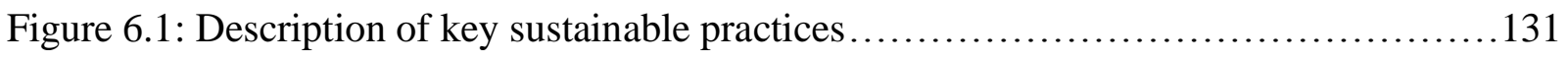




\section{List of Tables}

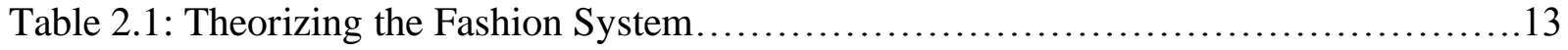

Table 3.1: Tools for sustainable fashion design....................................33-34

Table 3.2: Sustainable fashion Innovation framework...................................39

Table 3.3: Categorization of SSFD by impact and innovation level......................45

Table 3.4: Descriptions of the nine design dimensions of the parallel coordinate plot (PCP)...47

Table 3.5: Tool Archetype descriptions.......................................49-50

Table 4.1: Sustainable fashion definitions.....................................64-65

Table 4.2: Most commonly used sustainable strategies by the design entrepreneurs...........71

Table 4.3: Designer's sustainable design tool familiarity and use........................77

Table 4.4: Manufacturing and sourcing challenges faced by MSE design entrepreneurs......86

Table 5.1: Description of building blocks comprising the reDesign canvas.............107-108 


\section{INTRODUCTION}

The very essence of fashion is its transformative nature - its ability to be disruptive and make a statement. It is a communication tool and subject to symbolism. Yet, despite these characteristics that potentially could facilitate a transition towards system sustainability, fashion is instead underdeveloped in this area. For the purposes of this dissertation, sustainable fashion is therefore defined as "the profitable design, production, distribution, and end-of-life reuse, recycling, or disposal of fashion that supports circular systems, minimizes negative and maximizes positive impacts on both society and the natural environment".

While the key environmental and social issues of the industry have been discussed at large through the media and academia, awareness and education have been unsuccessful thus far in developing sustainability within the fashion industry. A frequent argument is the conceptual incompatibility of fashion and sustainability (Fletcher, 2010). Fashion thrives on inventiveness, innovation, creativity and transformation. But, how can an industry built on the very premise of change and innovation be so stunted when it comes to developing sustainability?

The fashion industry has developed one of the longest and most complicated supply chains, relying on resource-intensive material inputs (Fletcher, 2008). The associated negative environmental impacts reverberate throughout the entire life cycle of fashion from production, consumption through to disposal. The time consuming and resource intensive processes of fashion apparel draws on energy, water, toxic chemicals and non-renewable resources, while causing significant pollution problems to air, land and water (Allwood, Laursen, Russell, de Rodríguez, \& Bocken, 2008; Fletcher, 2008). The developments to production efficiencies within the supply chain are typically orientated around cost to maintain low-priced products. Maintaining low-prices typically results in reduced quality materials. Coupled with the movement of fashion production offshore, this has allowed for mass production of fashion apparel (Fletcher, 2008; Hethorn \& Ulasewicz, 2008). However, the current fashion production system has continued to be based on traditional practices and commercial technologies that are not sustainable (Scheffer, 2013; Tseng, Tan, \& Siriban-Manalang, 2013).

Developments to technologies and media within the field of communication also have had a major impact on the dissemination and influence of fashion. The world of fashion was once a 
coveted and secret world unbeknownst to most (Jenkyn Jones, 2011). Around the 1980s and 1990s fashion slowly began to be "in fashion." The widespread adoption of the Internet during the 1990s spawned unprecedented growth for the fashion industry. The democratization of information gave forth an ability to disseminate fashion trends to a wider audience in virtually real time (Jenkyn Jones, 2011). The Internet and social media were pivotal for creating "street style stars" and "influencers" with the advent of blogging platforms followed by Instagram. Street style photographers, bloggers and influencers alike showcase the fashions of stylish individuals on the street (Abidin, 2016; Çukul, 2015; Woodward, 2009) and prior to Instagram's takeover of fashion media, surveys have found the number of fashion blogs in existence to be between 2 and 3.8 million (Rickman \& Cosenza, 2007). The introduction of smartphones and apps such as Instagram, put the technological capabilities to capture and disseminate street style into the hands of mass society. These technological developments within communication platforms have enhanced the speed and breadth of fashion trend diffusion further inciting desire and participation. Instagram is now the fashion industry's favorite platform for engagement. The Internet has essentially altered how fashion is designed, produced, branded, marketed, and delivered (Çukul, 2015; Jenkyn Jones, 2011; Rocamora, 2017). A study conducted by Morgan and Birtwistle (2009) found that $16 \%$ of fashion consumers are heavily influenced by media in adopting new fashion trends. The 21 st century therefore witnessed a growth and popularity in fashion where fashion has become inextricably and increasingly linked to consumption.

It is clear the large-scale consumption of fashion apparel products has become problematic. Fashion products are not designed to deal with the negative issues surrounding consumption. When looking to define fashion, a discerning distinction exists where fashion constitutes clothing but not all clothing is considered fashion. Clothing, apparel, garments and any other term used to describe body adornments of various kinds have been used by humans as a form of expression and communication among social groups. Fashion, however, is unique as it includes all types of body adornments but is subject to an ever-changing form. Change is a seemingly necessary quality as supported by a review of the major body of analytical commentary that describes fashion as complex, diffuse, ephemeral, an aesthetic expression and a visible social behaviour (Horn \& Gurel, 1975; Nystrom, 1928; Robinson, 1961; Sproles, 1974; Wilson, 2003). Fashion has been described as design change for decorative purposes and the pursuit of novelty for its own sake (Robinson, 1961), while Horn (1975) defines fashion as a popular, accepted, and prevailing style at any given time that is a manifestation of collective behaviour. While differing 
perspectives exist in attempting to define fashion, it is clear change for aesthetic purposes is an intrinsic characteristic of fashion. For the purposes of this dissertation, two definitions of fashion will be used. In Chapter 2, which focuses on fashion theory, fashion is defined as a process signified by a prevailing style that is widely accepted by a group of consumers at a given time. For the remaining chapters of this dissertation, the term fashion is used more broadly in reference to products and cultural production. Fletcher's (2008) modern perspective is most applicable and is used for Chapters 3, 4, 5 and 6 where fashion is defined as clothing and people make up fashion which functions as a reflection and communication of our views within society, linking us to a time and space.

Fashion is a phenomenon that shapes and reflects societies and communities, their diversity, beliefs, values and cultures, is a personal expression, and signifies belonging to social groups. Fashion is far more than the tangible cloth formed into shapes. Fashion contrasts from clothing in its use and lifespan, associated symbols and meanings and are dictated by aesthetics, while clothing use and lifespan are dictated by functionality (i.e. appropriateness for activity, weather and wearability). Fashion is always about the "new" with the introduction of new trends every season. The "new" signifies we are "in fashion" and up to date and can therefore discard last season's clothing, perpetually feeding our desires of consumption for the "new".

Sustainable fashion products also neglect consumption issues. Sustainable fashion typically looks to technological solutions such as waterless dyeing or alternative environmentally friendly material sources. Designing sustainable garments within an unsustainable system is a piecemeal approach. Considerations of the fashion system must be included into the parameters of the design process such as supply chain, business practices and consumer use/disposal. Fletcher and Grose (2012) argue that offering consumers an alternative choice is not dealing with the deeper issues such as increasing consumption rates, patterns and behaviours. Consumption patterns and behaviours have thus been identified as a key issue in regard to sustainability (Chan \& Wong, 2012; Fletcher, 2012; Hoffman, 2012; Teunissen, 2013). The idea is to use fashion design as a powerful communication medium to influence consumers to more sustainable consumption behaviours. For the purposes of this dissertation, sustainable fashion is defined as the profitable design, production, distribution, and end-of-life reuse, recycling, or disposal of fashion that supports circular systems, minimizes negative and maximizes positive impacts on both society and the natural environment (Kozlowski, Searcy, \& Bardecki, 2018). 
Within the introduction and conclusion of this dissertation, there is a deliberate switch in terms to include responsible fashion alongside the use of sustainable fashion. The use of the term responsible is a considered response to the interviews conducted with sustainable fashion MSE design entrepreneurs. There was a consensus among the design entrepreneurs that the use of the term sustainable fashion is ambiguous, broad and has lost meaning. They preferred the use of the term responsible as they found it to be easily conceptualized by their stakeholders. The academic literature supports the use of the term responsible within sustainability studies and acknowledges the evolution of both the definitions, constructs and paradigmatic roots of responsibility and sustainability (Bansal \& Song, 2017; Montiel, 2008). Therefore, when the context is appropriate within the introduction and conclusion, the term responsible fashion is used.

Academics have challenged and redefined fashion in an attempt to reflect current societal views, but a contemporary refutation seems largely absent within the practices of the fashion industry. This doctoral dissertation research examines a re-conceptualization of the fashion design process by linking the practice of fashion design to consumers and their consumption behaviours. This research explores and develops theory, examines current sustainable fashion design practices and tools, and creates a sustainable design tool through action research that also builds upon previously developed theories and assumptions. While solutions to sustainability are becoming prevalent in the academic discourse, what is lacking is a wide-scale rebuttal to the ideologies driving the current fashion system.

This dissertation seeks to challenge the current status quo of the fashion design process for what it may become: change-centric solutions to sustainability. The purpose is to develop a framework for sustainable fashion design through a series of manuscripts. The first manuscript provides the arguments and contexts for a sustainable design framework through theory development. The second manuscript analyzes currently available fashion design tools intended to promote sustainability. The third manuscript investigates and analyzes interviews with microand-small enterprise (MSE) sustainable fashion designers to provide a better understanding of the constraints and opportunities in integrating sustainability into the design phase. The fourth manuscript is a case study where a case study helped to inform the development of a sustainable design tool, the re-design canvas. Working with a case study allowed for identification of leverage points within the design process. Leverage points are places within complex systems where small changes can impact the function of the system as a whole (Meadows, 2008). Analysis of this process informed the final design of the framework. The framework focuses on 
the fashion designer as a problem-solver and embeds the principles of sustainability at the core of the design process. Designers are creative and this framework attempts to apply that creativity to problem solving by expanding the limited aesthetic lens that designers currently work within.

\subsection{Research Objectives}

This research is designed to explore a reconceptualization of fashion design's role in driving innovative solutions for a sustainable fashion system. The central research question and objectives are outlined below.

Research question: In an era where fashion is one of the world's largest and most complex industries, promoting continuous growth and passive overconsumption, how can sustainable fashion MSE design entrepreneurs contribute to the creation of a responsible fashion system?

The following objectives have been developed to address the central thesis question.

Objective 1: To provide context for the development of a sustainable design framework through theoretical development of a fashion system theory (manuscript 1).

Objective 2: To analyze currently available sustainable fashion design tools to better understand their strengths, suitability, applicability and opportunities for improvement (manuscript 2).

Objective 3: Through semi-structured interviews, to examine and develop a better understanding of how MSE fashion designers currently work, the integration level of sustainability and use of sustainable design tools within design practice (manuscript 3).

Objective 4: To develop, test and refine a sustainable design tool through a real-world application in designing a fashion product (manuscript 4).

The research question and objectives seek to better understand how MSE fashion designers can change an industry deeply tied to the capitalist system (and with it, perpetual cycles of consumption and disposal) to reclaim fashion and dress as a meaningful tool for personal expression and cultural symbolism. For the purposes of this dissertation, sustainable fashion MSEs are defined as fashion firms with under fifty employees (United Nations, 2019) who are focused on sustainability. 


\subsection{Research Design}

The research design for this dissertation is shown in Figure 1. The research follows a manuscript-style dissertation, which entails a collection of manuscripts where the student is the principal author. This collection of manuscripts presents a coherent theme focused on a better understanding of sustainable fashion design practices within sustainable fashion MSEs. The dissertation consists of four manuscripts. Methods will be addressed independently within each manuscript.

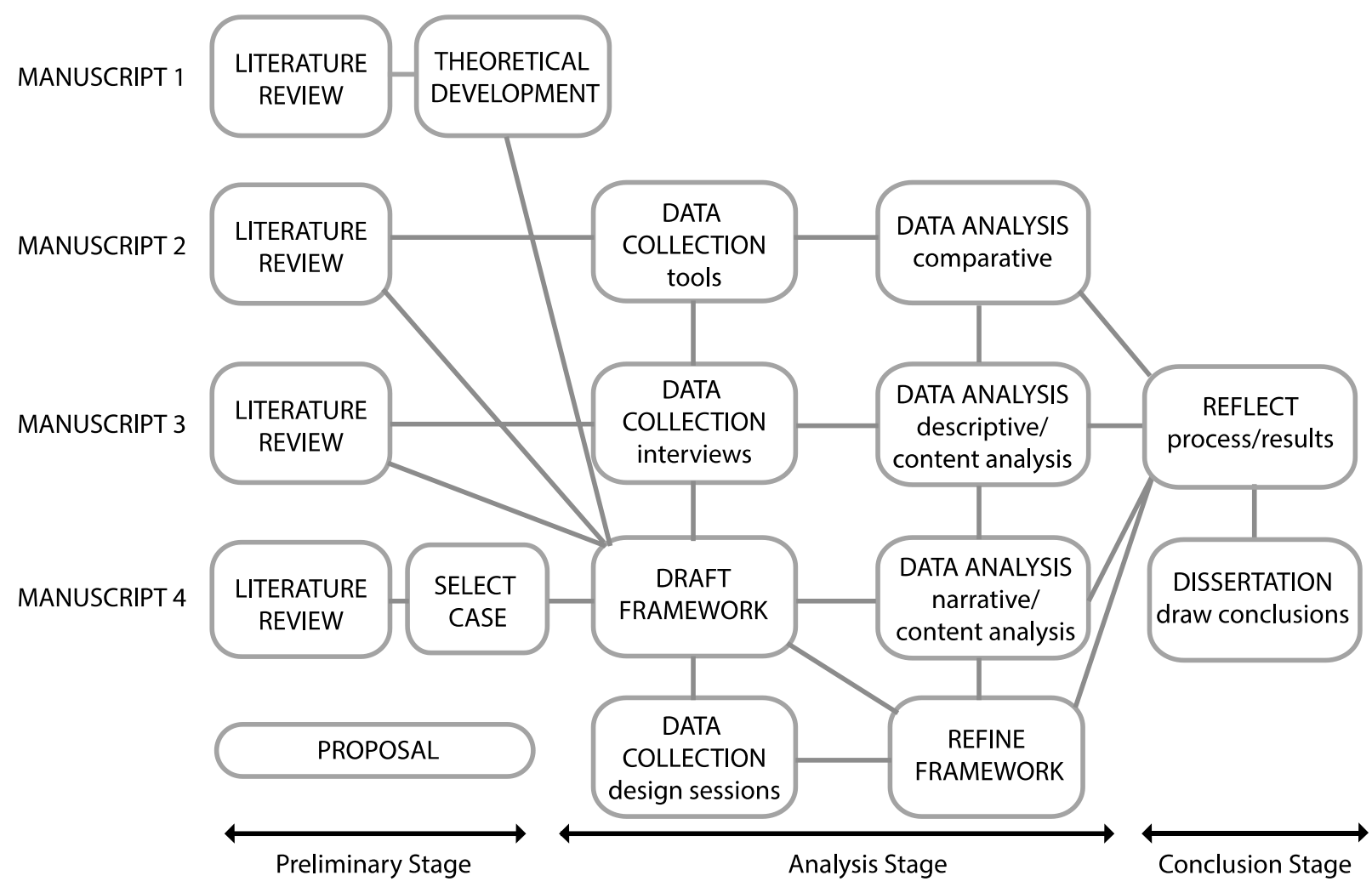

Figure 1.1: Research design

The first manuscript develops a conceptual model, forming the theoretical development for the following manuscripts. Manuscript two analyzes available sustainable fashion design tools and identifies the various sustainable fashion design strategies found within the content of the tools. The third manuscript interviews sustainable fashion design entrepreneurs who own and operate an MSE to identify their sustainable fashion design practices. The first three manuscripts contribute to the development of a sustainable fashion design tool for sustainable MSEs in 
manuscript four. The synthesis of results from all four manuscript address the research question and final conclusions.

\subsection{Outline of Chapters and Authorship Statement}

The research in this dissertation is organized into six chapters. Following the introduction, the foundational work is presented in Chapter 2. This chapter provides the theoretical foundation for the research study. Chapters 3 and 4 assess the existing design tools and explore the current landscape of sustainable fashion strategies used by MSE design entrepreneurs. Chapter 3 examines currently available tools for sustainable fashion design to determine their fitness for purpose. The analysis of the tools resulted in the development of two conceptual frameworks. Chapter 4 explores how current design-led sustainable fashion MSE designers integrate sustainability into their design practices and how that manifests throughout the rest of their business practices. The reDesign canvas introduced in Chapter 5 is the culmination of the research presented in the previous chapters. Lastly, Chapter 6 discusses and reflects upon the research study and future directions for research.

The dissertation has been written as a manuscript style. The remainder of the dissertation consists of the following chapters.

Chapter 2, Theorizing the Fashion System, reviews existing theories of fashion production and consumption, for the purpose of establishing a conceptual model to support subsequent research and tool design. This conceptual model presents a holistic and systems thinking approach for the development of a sustainable fashion system. Applying a model of systems thinking provides a better understanding of how sustainable fashion design practices can affect consumption, business practices and design tool development. This chapter is currently under review for publication in Fashion Practices. Authorship statement: Anika Kozlowski conceived of the concept, developed the framework and original draft preparation. Figures were developed by Anika Kozlowski and Michal Bardecki. Reviews and editing were completed by Michal Bardecki and Cory Searcy.

Chapter 3, entitled Tools for Sustainable Fashion Design: An Analysis of their Fitness for Purpose, examines existing design tools developed specifically for sustainable fashion designers. This research led to the creation and proposal of two conceptual frameworks: an innovation framework and five-dimensional model of sustainable fashion. Using the frameworks to analyze the tools and sustainable strategies within the tools resulted in the identification of three tool 
archetypes: 1) Universal, 2) Participatory and 3) Assessment. Authorship statement: Conceptualization of the research and original draft preparation was undertaken by Anika Kozlowski. The methodology was developed by Anika Kozlowski, Michal Bardecki and Cory Searcy. Review and editing were performed by Cory Searcy and Michal Bardecki. Visualization and figures were created by Anika Kozlowski and Michal Bardecki.

This chapter has been published as follows:

Kozlowski, A., Bardecki, M., \& Searcy, C. (2019). Tools for sustainable fashion design: An analysis of their fitness for purpose. Sustainability, 11(13), 3581.

\section{Chapter 4, A Case Study of Sustainable Fashion MSEs and their Sustainable Design}

Practices seeks to comprehend how sustainable fashion design entrepreneurs who own and operate an MSE integrate sustainability into their design practice. This study looked at designer's knowledge, use of sustainability practices and tools. Themes and patterns into the types of sustainability strategies used and challenges were identified through a qualitative content analysis process. Authorship statement: Anika Kozlowski conceived of the concept, developed the method and original draft preparation. Review and editing were performed by Michal Bardecki and Cory Searcy.

Chapter 5, The reDesign Canvas: Fashion Design as a Tool for Sustainability was a culmination of prior research to create a sustainable design tool. This tool aims to support design entrepreneurs in developing sustainable fashion enterprises. Informed by design thinking and systems thinking, the canvas was developed based on an in-depth review of the academic literature and the collection of qualitative data. The final version of the canvas is based on 12 building blocks that a design entrepreneur would encounter in building a sustainable fashion brand. Authorship statement: Anika Kozlowski conceived the concept of the reDesign canvas and prepared the original draft. The methodology was developed by Anika Kozlowski, Michal Bardecki and Cory Searcy. Review and editing were performed by Cory Searcy and Michal Bardecki.

This chapter has been published as follows:

Kozlowski, A., Searcy, C., \& Bardecki, M. (2018). The reDesign canvas: Fashion design as a tool for sustainability. Journal of Cleaner Production, 183, 194-207. 
The conclusion makes up Chapter 6, and summarizes the research results, discusses the results and progress made in relation to the research question and considers the overall research goal. This chapter includes a series of reflections and recommendations for future research regarding MSEs designers and their role in the development of a sustainable fashion system. Authorship statement: Anika Kozlowski wrote Chapter 6 in traditional thesis style, where the ideas within are a summary of the $\mathrm{PhD}$ research and direction for both future work and publication. 


\title{
2. THEORIZING THE FASHION SYSTEM
}

\begin{abstract}
Fashion is a largely practice-based discipline and theory that uses a broad multi-disciplinary perspective is limited within fashion studies. Fashion sits at the nexus of differing social, psychological, economic, and cultural contexts and has been studied through a variety of lenses. These lenses often offer significant insight but do not account for the breadth of the multidisciplinary nature of fashion. Fashion has been visualized from the theoretical perspective of many individual disciplines. We seek to situate the academic study of Fashion within an inclusive conceptual model by considering and conflating multiple theories from various disciplines. The single conceptualization seeks to represent and outline how the fashion system currently is constructed. The foundation of the model is expressed as a series of counterpoints developed from existing theory. The four distinct aspects and counterpoints are: (1) Agency: Creator - Consumer; (2) Supply Chain: Production - Consumption; (3) Merchandising: Brands Cues; (4) Motivation: Art - Commerce. These form the basis for a unified conceptual model representing the various aspects of the fashion system. Among these dimensions and counterpoints, the central role of design and the designer is recognized as a foundational aspect. The model provides a framework that can be applied to the diversity of areas of research interest in the discipline.
\end{abstract}

\subsection{Theory in Fashion}

The roots of academic interest in fashion are diverse. A survey of university programs in Fashion $^{1}$ reveals examples with ties to each of Creative Arts, Fashion Studies, Social Sciences, Home Economics, Textile Science, Merchandising, Marketing, Curation and Costuming. Furthermore, fashion research is embedded in a variety of disciplines: scholars in areas as diverse as Business, Media Studies, Sociology, Engineering and Psychology conduct fashionoriented research. Much of the research is approached from the perspective of one academic discipline. Despite the fact that fashion research inherently crosses disciplinary boundaries,

\footnotetext{
${ }^{1}$ We capitalize "Fashion" when referring to the discipline.
} 
broad multi-disciplinary perspectives are under-represented in the literature. Kaiser, Nagasawa, and Hutton (1989, 1995), and Nagasawa, Kaiser, and Hutton (1996) a three-part series are one of the few examinations of fashion rooted in the discipline of Fashion studies, incorporating a holistic approach to develop a theory of fashion. Using symbolic interactionism and following the scientific model of inquiry, the researchers draw upon multiple disciplines to formulate their theory that seeks to explain the dynamic phenomenon that is fashion.

Fashion has suffered from the perception it is not an area of serious academic research (Harris, 1995; Jarvis, 1998). In part, this can be attributed to the lack of a sound theoretical foundation for the discipline. It has been widely noted that Fashion has been under-theorized (e.g., Doherty, 1999; Entwistle, 2000; Skjold, 2008; Granata, 2012; Faerm, 2013). Indeed, as is common for a discipline which is in large part practice-based, Fashion theory has largely remained rooted in the perspectives of other disciplines where a variety of theories have been applied (Barnard, 2014; Barnes, 2013; Lynch \& Strauss, 2007; Miller, McIntyre, \& Mantrala, 1993; Pannabecker, 1997). For example, in reviews of fashion change, Lynch and Strauss (2007) identify 35 theories which have been used and Sproles (1985) identifies 20 different theoretical approaches just from "behavioral science". In most cases the theoretical approaches in Fashion have been appropriated from related discipline areas and have been relatively narrowly focused, rather than the discipline as a whole (Barnes, 2013); we believe this has impeded the development of a unified theory.

One may broadly divide the range of theoretical approaches to understanding fashion based on their focus (and origins) into the following categories: social/cultural, psychological, aesthetic, marketing/economic, and historical (Figure 2.1). In the figure, examples of theories are provided within each area- the divisions are somewhat arbitrary in that some theories overlap fields and the lists are not meant to be comprehensive. 


\section{THEORY IN}

FASHION

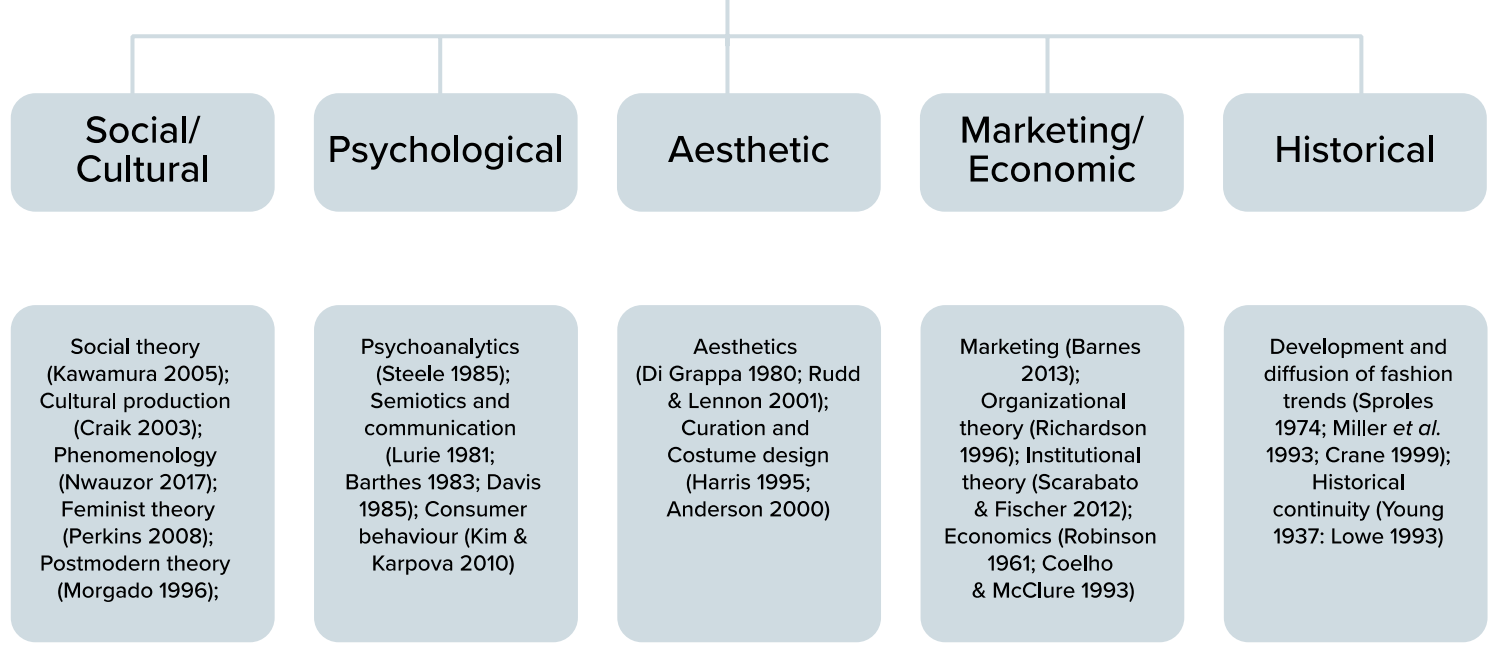

Figure 2.1: Theory in Fashion

We take the fashion system as incorporating the set of all aspects of the production, creation, business and consumption of clothing, attire, apparel, and garments, all of which functions as a system within larger social, cultural and economic contexts. In this paper we present a conceptual model that embraces the diversity of academic modes to the study of fashion. This is accomplished by considering and conflating a variety of theoretical approaches into a unified perspective of the fashion system.

By bringing together multiple perspectives, we seek to develop a conceptual model to explain and understand the nature of the fashion system. Many efforts to characterize the fashion system have focused on just one aspect of the system, such as the physical and/or communication linkages in production and consumption (e.g., Almila, 2016; Hauge, Malmberg, \& Power, 2009; Loschek, 2009; Pan \& Holland, 2006). These fragmented approaches do not adequately recognize the multi-dimensional nature of fashion. We view fashion as a multidimensional object of research and believe theory must do the same. The fashion system involves highly interrelated connections between the various elements of the production system for clothing and the configurations of distribution and exchange, as well as consumer demand 
and behavior (Leopold, 1993; Webster, 2016). We seek to bring a comprehensive range of perspectives to bear on theory in fashion.

Our purpose is not to provide a comprehensive survey of the theoretical underpinnings of fashion, but rather to draw together disparate strands of theoretical interest within a coherent model. We start with five aspects, four of which are articulated through the nexus of a pair of counterpoints developed from existing theory.

\subsection{Aspects}

Five aspects guide our approach.

Aspect 1: The fashion system incorporates social and cultural processes, which are its foundation.

Aspect 2: The fashion system also embodies important economic and financial aspects; a comprehensive theory of the fashion system needs to embrace these.

Aspect 3: Much of the fashion system is behavioral at its foundation; psychological theory can aid in explanation and understanding.

Aspect 4: Garments, to some greater or lesser extent, articulate both art and utility; the fashion system embraces both.

Aspect 5: At the heart of all fashion is design and the designer.

For each of the first four aspects we argue that there is a pair of diametric counterpoints that provide the fundamental theoretical underpinnings (Table 2.1). Diametric counterpoints are used to represent the broad range found within each aspect. For example, Aspect 4 signifies the motivation and the broad spectrum of categorization that exists in fashion from art to commercial.

Table 2.1: Theorizing the Fashion System

\begin{tabular}{|c|l|c|}
\hline Aspect & & Counterpoints \\
\hline 1 & Agency & Creators $\leftrightarrow$ Consumers \\
\hline 2 & Supply Chain & Production $\leftrightarrow$ Consumption \\
\hline 3 & Merchandising & Brands $\leftrightarrow$ Attitudes and Cues \\
\hline 4 & Motivation & Art $\leftrightarrow$ Commerce \\
\hline 5 & \multicolumn{2}{|c|}{ Design and the Designer } \\
\hline
\end{tabular}


Aspect 1 is expressed as Agency-that is incorporating the Creators and Consumers of fashion. Aspect 2, expressed as the Supply Chain, involves Production and Consumption as distinct aspects of the economic, social and cultural systems. Aspect 3 is expressed as Merchandising and is conveyed through fashion Brands and the Cues (which involve Aspect attitudes) related to consumer behavior. Aspect 4 is expressed as Motivation and its counterpoints are Art and Commerce-these acknowledge the importance of both the creative arts and business as drivers of fashion. Aspect 5 links the counterpoints in each of the other dimensions. The role of design and the designer form the foundation; for this aspect identifies design and the designer as playing pivotal roles in linking the counterpoints in each other dimension.

We recognize that these dimensions are interrelated. Although they do not exist separately within the fashion system, we discuss each in turn for the sake of clarity. For each aspect, we make a case by employing apposite examples of the theoretical foundations which illuminate the nature of the aspect.

\subsubsection{Aspect 1: Agency}

There is a diverse range of social- and culturally-based theories which have been, or could be, used to inform the understanding of fashion (Figure 2.1). Although there are substantial differences in foundations and methodology, at the core of each is an effort to illuminate how societies and cultures develop and function, and how individual, group, and organizational behavior is influenced by the social and cultural milieu in which they operate (Harrington, 2005; Smith \& Riley, 2011). These theories link to sociology, anthropology and other disciplines.

We follow the Oxford Dictionary in identifying agency as "a thing or person that acts to produce a particular result” (“Agency,” n.d.). At its core, fashion has two principal forms of agency: Creator and Consumer. Each embodies the differences between corporate behavior and individual expression, and market versus social norms.

Our interpretation of fashion agency is informed by Bourdieu's theory of social practice. Pierre Bourdieu arguably has been the most important theorist of culture and society in the latter half of the twentieth century. Bourdieu was interested in analyzing the relationship between social structures and individual actions in the production and consumption of culture. His theories have been highly influential, albeit controversial, and have been widely applied, often in disparate ways (they are often not entirely coherent). 
Bourdieu produced a large body of work that is difficult to compress. Central to Bourdieu's ideas $(1986,1977)$ is the interplay of field, capital and habitus (Figure 2.2). Bourdieu sees society as a multidimensional space comprised of a diversity of "fields" - a field being the network of structured social and institutional relationships (e.g., within social groups, communities, workplaces) where various agents (e.g., individuals and institutions) express and reproduce their dispositions and where the distribution of power (economic, social, cultural and symbolic "capital") affects access to the specific rewards that are at stake (Bourdieu \& Wacquant, 1992; Navarro, 2006). Capital can be drawn on and accumulated to establish, maintain and/or enhance one's position in the social order (Bourdieu, 1986). The unequal distribution of capital is seen to create an arena of relational positions based on the struggle around production, accumulation, circulation and possession of goods, services, knowledge and status. The agents are imbued with "habitus" - the subjective system of "lasting dispositions, or trained capacities and structured propensities to think, feel and act in determinant ways, which then guide them" (Wacquant, 2005, p. 316). Thus, the habitus is an aggregate of an agent's resources, and the amount and type of capital (cultural, social and economic) that an agent possesses.

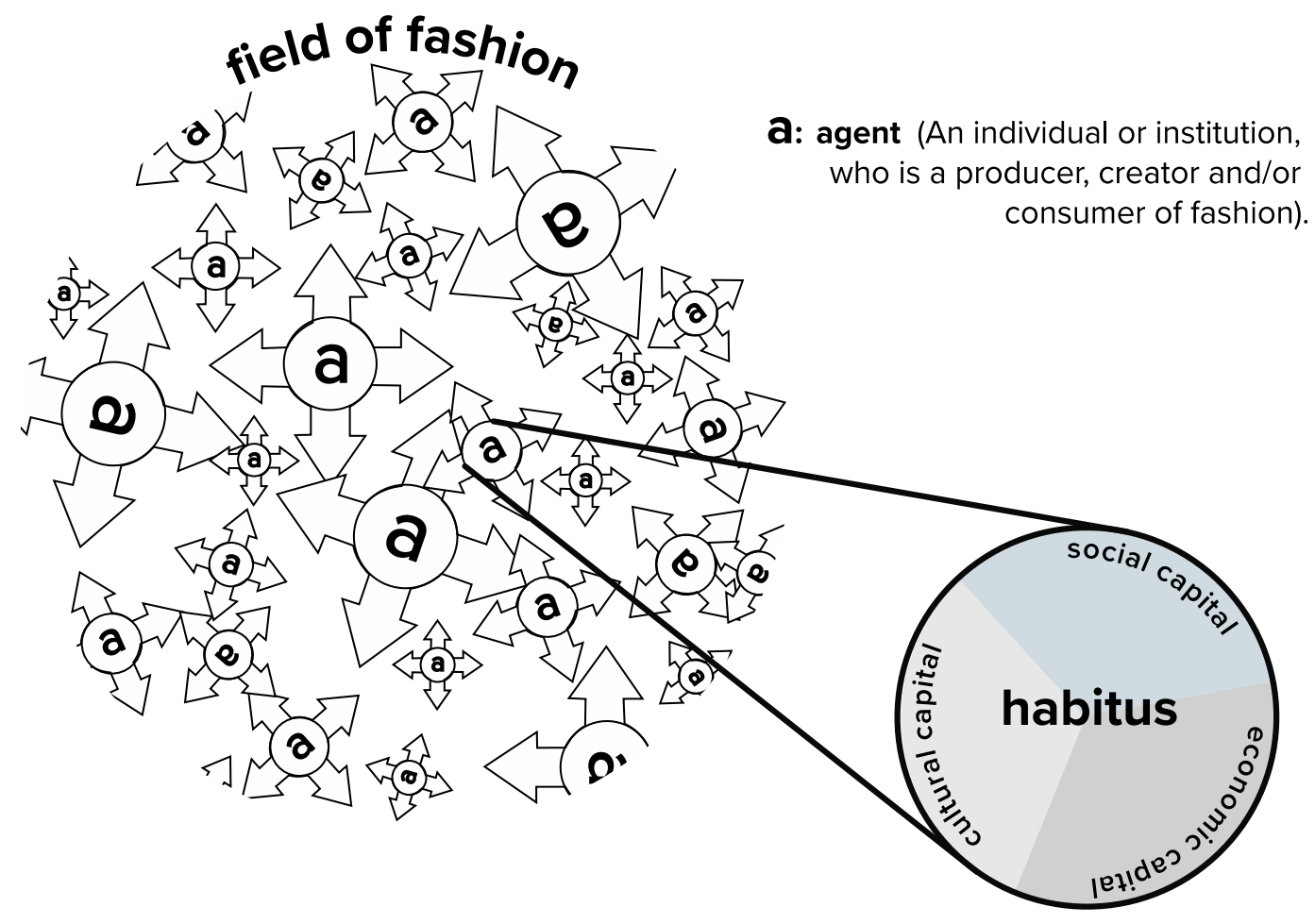

Figure 2.2: Agency in Fashion (following Bourdieu's Theory of Social Practice) 
An agent's habitus plays a role in the symbolic capital and power an agent possess in a field. Agents differ in a field through their symbolic capital and power relations. Social practices are revealed through agents interacting in a field. Figure 2.2 illustrates social practices through the interactions (arrows) of agents and their symbolic capital and power, as influenced by their habitus, in the field of fashion.

At their core Bourdieu's theories rely on the examination of agency: the nature of the agents and their roles, their social interactions, and relationships of power within the many fields in society. Fashion is not exempt from these larger social influences. Indeed, Bourdieu (1993a) did turn his scrutiny to fashion and his work has had an impact in the discipline (Barron, 2007; Entwistle, 2000, 2009; Entwistle \& Rocamora, 2006; Pedroni, 2015; Rocamora, 2002). This work has focussed attention on both creators and consumers of fashion. One needs only to consider the various modes of design in fashion: domestic (for family), haute couture (for elites), mass fast fashion (low cost, for mass consumer), ready-to-wear (higher cost, symbolic brands), bridge brands and subculture/street style (for self or group) to recognize that the agency through which these forms of fashion are delivered do differ. Likewise, within fashion, both "creators" and "consumers" encompass a variety of different agents including individuals, groups and organizations (Entwistle, 2002; Pettinger, 2004).

\subsubsection{Aspect 2: Supply Chain}

The pivotal observation related to the economic and financial character of the fashion system is the central role of the linkages between production and consumption (supply and demand). In part, the fashion industry is structured, and continues to re-structure itself, to maximize growth and profits through greater production and consumption. On the other hand, there is the recognition, dating back to Adam Smith, that "Consumption is the sole end and purpose of all production; and the interest of the producer ought to be attended to only so far as it may be necessary for promoting that of the consumer" (Smith, 1776/2012, pg. 658).

Theory of systems of provision (SOP), as introduced in the work of Ben Fine (Fine, 2002; Fine \& Leopold, 1993), is particularly useful for our purposes. SOP embodies the idea that consumption and production are inextricably intertwined and that they have evolved through the centuries of fashion change (Figure 2.3). This figure sees production and consumption as progressively more intertwined as technological innovations and globalization allow for greater interlinking of and more rapid change in production and consumption. Design is shown as the 
core of the system linking production and consumption. The SOP approach differs from other theories in that it expands the concept of fashion production to include cultural intermediaries and consumption. Fashion is also a cultural object that embodies the social and cultural values of a society at a particular time and place. Intermediaries such as bloggers, editors, buyers,

merchandisers, marketers, supply chain managers and photographers play an essential role as the “connective tissue" between supply and demand (Fine \& Leopold, 1993).

\section{Century}

$21^{\text {st }}$

Fast fashion

IT innovations

Globalization

Democratization of fashion

$20^{\text {th }} \quad$ Pluralization of style

Dominance of retail

Outsourcing of production

Ready-to-wear

19 ${ }^{\text {th }} \quad$ Technological advances

(sewing machine, band knife)

Designer fashion

First Fashion magazines

\& Department stores

$18^{\text {th }} \quad$ Handicraft production

Charles Worth (father of haute-couture)

$17^{\text {th }} \quad$ Bespoke production

Craft production

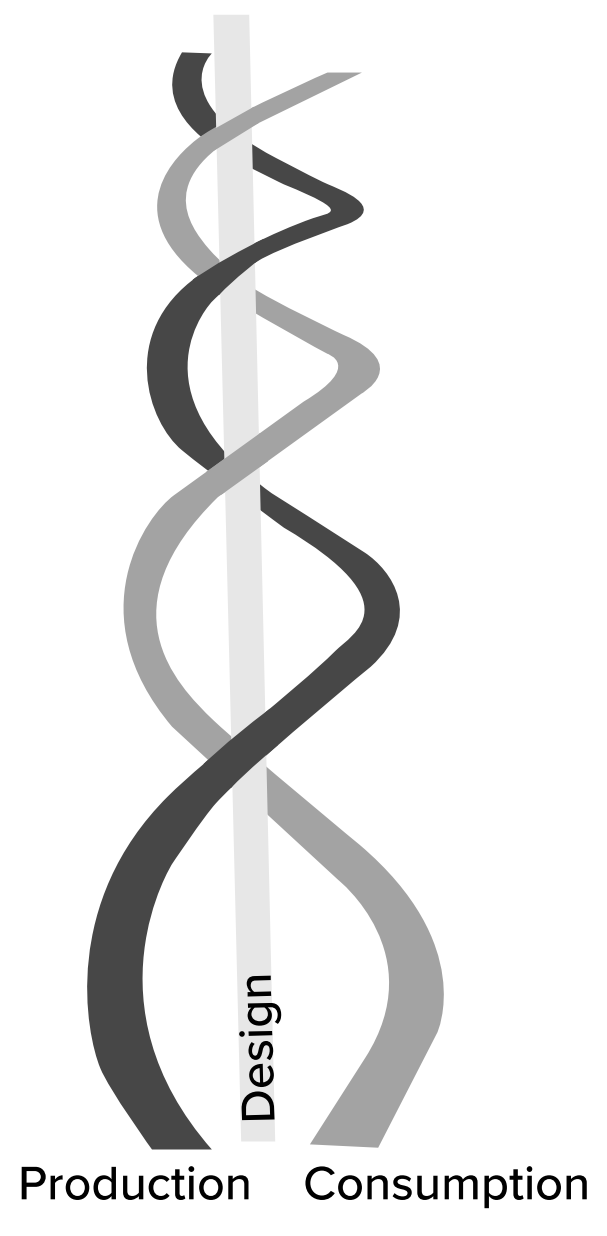

Figure 2.3: Systems of Provision in Fashion

Figure 2.3 demonstrates the intertwined relationship between fashion production and consumption. Design has always been a central element that links the two processes together. The left-hand side lists some key events that had a significant impact on the relationship between fashion production and consumption. As the industry developed, it has become less clear who the producers and consumers of fashion are. The intertwining relationship represents the shift 
from a modernist consumer for social class to someone who can both be a producer and consumer of fashion for identity affirmation, acceptance and uniqueness. Postmodernism views the terms producer and consumer to have multiple meanings (e.g., cultural production and physical product manufacturing).

SOP emerged as a critique of so-called horizontal approaches to consumption and a dissatisfaction with methodologies used to examine commodity flows that favored either production or consumption (Evans, 2011; Fine \& Leopold, 1993). Brooks (2015) suggests the importance in "look[ing] at the process outside of the borders of linear commodity chains, backwards to the historical events that determine regimes of production and consumption, while also combining cultural and political approaches to establish how value is produced in commodities"(p.37). SOP has usually been rendered in a historical context through an effective and clear engagement with production of value and historical materialism (Brooks, 2015; Fine, 2002).

SOP has been applied to fashion (Briggs, 2013; Fine, 2002) providing a broader lens through which changes in patterns of consumption and socio-economics are easily recognized (Evans, 2011). The SOP approach recognizes the role of both producers of fashion and customers as active agents. Over time, the relationships have become more entwined and change more rapidly.

The appeal of the SOP approach is the holistic lens it applies to the study of production and consumption in fashion, taking into account the social and economic organization of commodities, goods and services. Unlike much of the literature in fashion where production and consumption are addressed separately, adoption of an SOP approach allows for a perceptive understanding of how demand for fashion is created and managed by studying the inter-relations of consumption and production. In his analysis on the culture of production within fashion, Briggs (2013) emphasized the ability of the SOP to question both the ideas that consumer choice drives the marketplace, and that such behavior is driven by the activities of producers. Fashion production and consumption exist as a dialectical relationship where neither are discrete entities that operate independently, with either one being the primary determining agent (Briggs, 2013).

The commercial character of culture has eroded any distinctions between culture and practical life. Postmodernism is attributed by many to be the stimulus in the shift in mass culture and its subsequent influence on consumption (Adorno, 1991). Consumption shifted from an 
activity based primarily on functionality of needs to one influenced by the plasticity and volatility of desire. The idea of mass producing and consuming culture introduced a new dimension to culture (which includes fashion) that is no longer dictated by social hierarchy (Adorno, 1991). As a result, markets broaden, product turnover speeds up and concepts such as planned obsolescence are introduced thereby redefining patterns of consumption which now exist in a state of flux (Friedman, 1994).

Other theories characterize fashion as linear linkages of consumption and production portrayed as flow diagrams of supply chains, value chains and life cycles. SOP considers linkages associated with production and consumption to include considerations such as the emotional needs that might be satisfied through the constant turnover of fashion products. SOP represents an analytical approach that moves away from descriptive catalogues of fashion change to include human behaviors and motivations. By establishing context and considering behaviors, we can better understand how value is created and attributed to fashion products.

\subsubsection{Aspect 3: Merchandising}

Consumer research has long considered understanding the relationship between attitudes and behaviors as essential (LaPiere, 1934). The approach most widely adopted in examining the attitude-behavior relationship is the theory of reasoned action, which proposes that behavior arises from intention, which in turn derives primarily from subjective norms and attitudes toward the action (Fishbein \& Ajzen, 1975).

These ideas resonate in several different approaches to understanding brands and consumer behavior. For example, Oliver (1999) incorporates both attitudinal and behavior aspects of loyalty in a four-stage model consisting of cognitive (i.e., perception of attributes), affective (attachment and attitude formation), conative (commitment), and action (i.e., behavior). Similarly, the four phases of Keller's (2001)widely-cited concept of "brand resonance" include attitudinal and behavioral aspects:

- Behavioral loyalty: the observable repetition of a purchase behavior

- Attitudinal attachment (“commitment"): differentiates genuine loyalty to a brand from other forms of repeat purchases which include habit, inertia and spurious loyalty (Beatty \& Kahle, 1988; Dick \& Basu, 1994).

- Sense of community: i.e., where consumers identify with other people associated with the brand. 
- Active engagement: i.e., behavior beyond those resources expended during purchase or consumption of the brand.

The idea that consumer behavior can be driven both by brand loyalty and the contextual cues related to "habit" and that one needs to understand both to explain purchasing behavior continues to be explored (Lemmerer \& Menrad, 2017; Liu-Thompkins \& Tam, 2013; Tam, Wood, \& Song, 2009).

Habits may be defined as those "behavioral disposition[s] that [are] exercised frequently and in which responses are triggered directly by contextual cues" (e.g., setting, prior actions) (LiuThompkins \& Tam, 2013,pg. 22). There are strong psychological (Duhigg, 2012; Finlay, Trafimow, \& Villarreal, 2002; Robinson \& Berridge, 1993) and neurological aspects (Erk, Spitzer, Wunderlich, Galley, \& Walter, 2002; Schaefer \& Rotte, 2007) to the development and activation of habits. Research has indicated that with routine repetition of action, shifts occur in the cognitive and neurological mechanisms that guide behavior (Neal, Wood, Labrecque, \& Lally, 2012; Neal, Wood, \& Quinn, 2006; Wood, Tam, \& Witt, 2005). When behaviors are repeated, habits are formed that reflect cognitive, neurological and motivational change (Neal et al., 2006; Wood, Quinn, \& Kashy, 2002; Wood et al., 2005). Rewards support habit formation (Wood \& Neal, 2009) which are activated automatically by contextual cues that co-occurred in the past (Lally, Van Jaarsveld, Potts, \& Wardle, 2010; Neal et al., 2006). Once habits are initiated, they can proceed independently of intentions and with minimal conscious control (Ji \& Wood, 2007; Neal et al., 2006; Wood \& Neal, 2009).

Cue utilization theory (Easterbrook, 1959) is based on the concept that an array of cues can serve as surrogate indicators for consumers of a product's worth. It has been used to evaluate consumers' perceptions of brands. Cue utilization theory has proven to be a useful basis for the exploration of brands and consumer behavior (Collins-Dodd \& Lindley, 2003; Dawar \& Parker, 1994; Richardson, Dick, \& Jain, 1994). Considerable research has explored the relationship between habit and brands (e.g. Beatty \& Kahle, 1988; Liu-Thompkins \& Tam, 2013; Robertson, 1976). Brand involvement ("brand commitment") relates to the degree of a consumer's emotional attachment to a specific brand (Beatty \& Kahle, 1988; Wang, 2002); these are resistant to change (Dholakia, 1997) reflecting both attitudinal attachment and habit. Brands can encourage consumers' behavior to become progressively more routine and driven by habit (Oliver, 1999). Through design brands can activate the cue-routine-reward loop (Figure 2.4). 


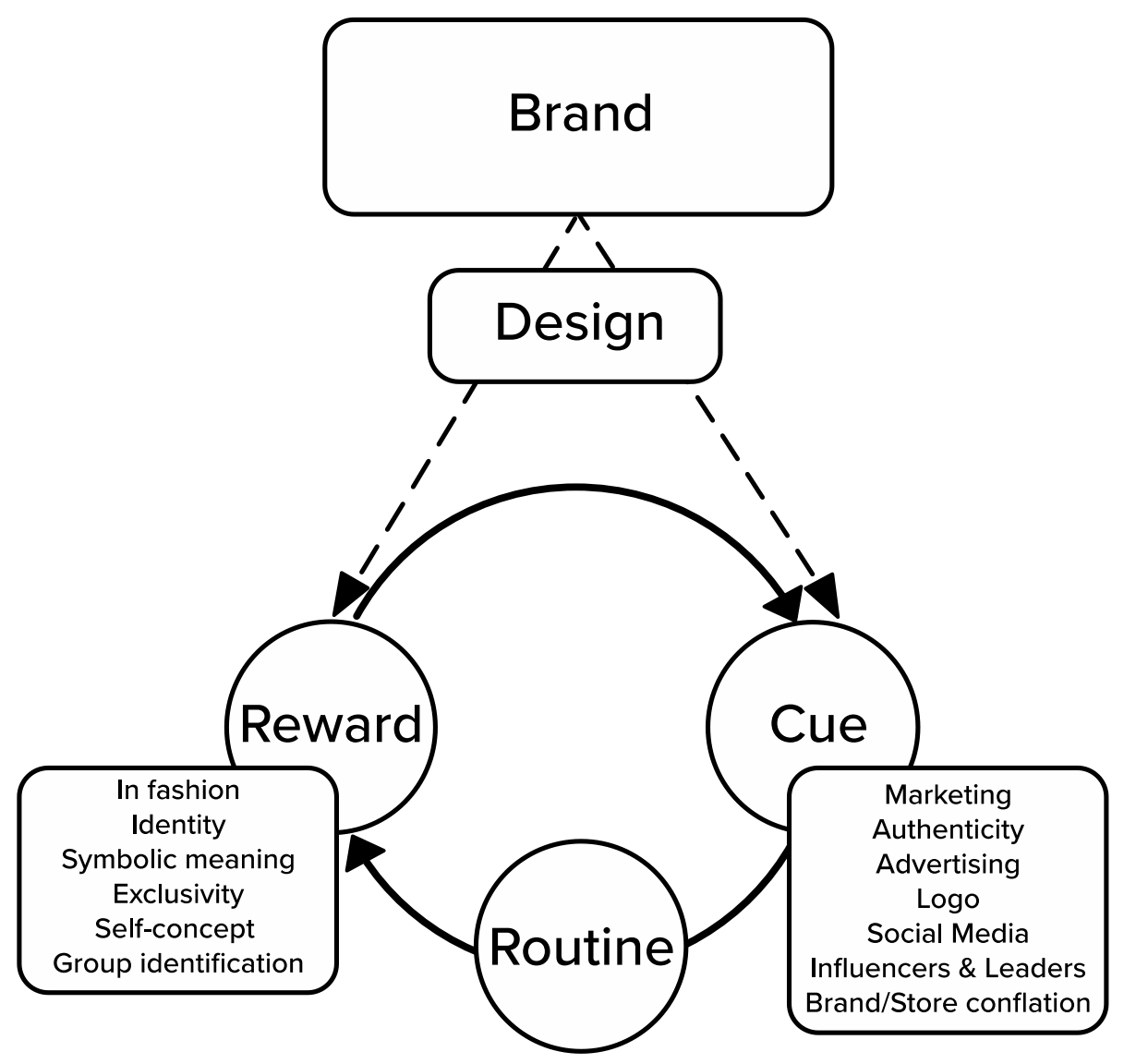

Figure 2.4: Merchandising in Fashion

The fashion industry has been built on the idea of craving and desire; whether it be a social class or group, fashion leadership or the symbolic values of a brand, desire in its various forms motivates consumption. Fashion trends function to create new products or looks to desire. While technology has sped up the trend cycle and created greater availability and immediacy of fashion trend information, globalization and offshore production coupled with fast fashion have lowered costs, prices and created accessible fashion. Essentially, the fashion industry functions in a manner that creates the ideal conditions or context and cues to form fashion habits. This further predisposes the formation of fashion habits as social practices shape context and cues which, when validated through consumer behaviors, reinforce social practices. For example, social media platforms like Tumblr and Instagram gave rise to the "influencer" persona which fashion brands have largely employed as a new cue mechanism for fashion consumption. 
Fashion involvement through multiple channels (e.g., the mall or social media), also carries semantic ambiguity as fashion consumption refers to both culture and/or object. For example, a fashion influencer is a producer of cultural fashion and a consumer of cultural fashion objects. The Internet has created a space for multi-channel multi-meaning fashion production and consumption. As a self-referential system, an increase in cues increases consumption, increasing demand and supply. A culture of consumption eagerly exploits the virtual and physical spaces of the world in order to produce low- cost cheap objects and cultural images to sell large volumes of clothing at an increasing speed. Placing high value on consumption in its various forms, shapes social values and can influence attitudes.

As cues proliferated, so too did involvement in fashion as demonstrated through increased production and consumption of fashion apparel. This has affected motivation, attitude and habit consumption behaviors, largely shaped by the overwhelming frenetic pace of the industry today. The fashion field today affords circumstance and agency, where fashion leadership is no longer reserved for industry insiders and elite, as anyone with an Instagram account has the potential to become a fashion leader.

Fashion leadership has been studied extensively as it plays a key role in the diffusion of new fashions. Fashion influencers are more attuned to fashion trends, learning about new fashion trends and purchasing them earlier than the average buyer (Goldsmith, Freiden, \& Kilsheimer, 1993). Apps like Instagram, the rise of street style, and the growing importance of influencers has also functioned to increase obsolescence. Instagram has reinforced the unwritten rule of never wear the same outfit twice. Certain fashion bloggers such as Man Repeller, Tommy Ton, or Susie Bubble have become as influential as magazine editors in shaping trends, commentary and sharing the latest news and images of the most recent trends (Grinberg, 2013). A recent study found that the more a consumer identifies and has a favorable attitude towards a fashion blogger the more likely they are to purchase items showcased by that blogger (Hahn \& Lee, 2014).

Social media also enables personal style documentation. This has established itself as a central form of fashion blogging, asserting itself as a "key space for the production and circulation of fashion discourse... by bringing together the old technologies of the self- fashion and photography and the new technologies of the self- the blog and the screen, personal fashion blogs can be seen as a privileged space of identity construction" (Rocamora, 2011, pg. 409-410). Fashion content can now be documented and produced by anyone. Technological developments 
within communication platforms have enhanced the speed and breadth of fashion trend diffusion, further inciting desire and participation. Further encouraged by low prices and advertising campaigns, consumers tend to speed up consumption of fashion (Birtwistle \& Moore, 2007). The breadth of communication media support fashion as a social practice. For example, Instagram has over 600 million users, more than 300 million of whom use Instagram every single day, share an average of 95 million photos and videos per day and like 4.2 billion posts daily (Instagram, 2017). Fifty percent of Instagram users follow at least one business, sixty percent say that they learn about a product or service on the platform, and seventy-five percent of users take action such as visiting a website, after looking at an Instagram advertising post (Instagram, 2016). Using social media for fashion information is an ideal conduit for forming habit consumption behaviors.

\subsubsection{Aspect 4: Motivation}

The entire system of fashion is highly disparate. Two often incongruent motivations have been seen to guide fashion: the realms of art and that of commerce (Barnard, 2014; Bourdieu \& Delsaut, 1975; Entwistle \& Rocamora, 2006). Although the fields do intersect, the fashion system embraces the dialectic of art and of commerce (Figure 2.5). Theorists have argued that the dominant perspectives are distinctive and that these empower and constrain their actors differently. This affects their "embedded agency" (Greenwood \& Suddaby, 2006; Scaraboto \& Fischer, 2012; Seo \& Creed, 2002).

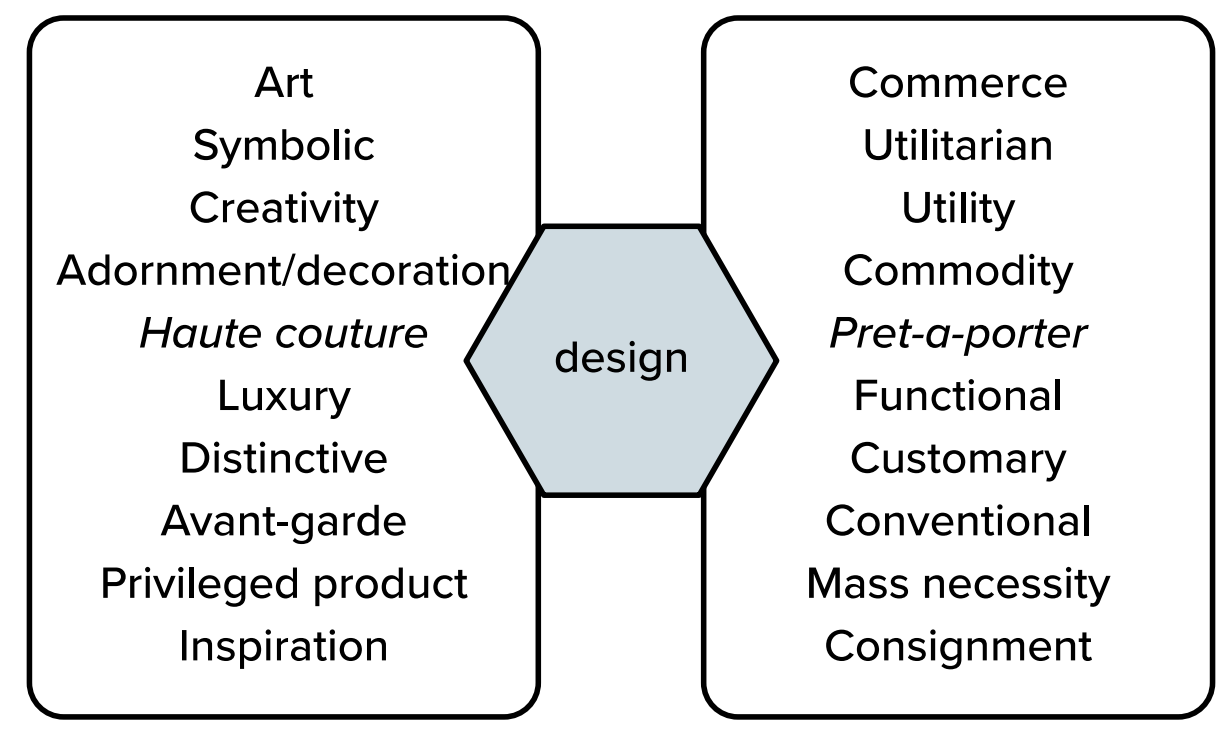

Figure 2.5: The Dialectic of Motivation in Fashion 
Fashion can exist without a commercial market. Indeed, some would see fashion and art as "similar if not identical impulses" (Martin, 1999, pg. 111) distinct from commercial pressure. Perhaps it is only in museum or gallery collections that fashion objects may "become pure aesthetic artefacts" (Choi \& Lewis, 2018, pg. 14). Designers such as Rei Kawakubo and Iris van Herpen produce garments that sit within the realm of art, frequently showcased in exhibits rather than worn as everyday fashion garments.

Much of fashion is peripheral to the "aesthetic economy" (Entwistle, 2009). Although often seen as the production of "pure" art (Rocamora, 2002), even haute-couture houses are not distanced from the commercial—recognizing that there always needs be some reference of function beyond decoration/ornamentation (Robinson, 1958). Likewise, commercial products in fashion incorporate the aesthetic, cultural and economic values. The meshing of fashion with wider aesthetic matters has not been fully settled (Craik, 2009; Mackrell, 2005; Troy, 2003), and there is a generally limited literature regarding the influences on aesthetic theory on fashion (Evans, 2003; Hollander, 1992; Nicklas, 2014; Rudd \& Lennon, 2001).

Again, through his "field analysis" (Bourdieu, 1993b; Bourdieu \& Delsaut, 1975) provides a framework for understanding. Bourdieu's "fields" draw attention to the relations which exist within the "social microcosms, separate and autonomous spaces, in which works are generated" (Bourdieu, 1996, pg. 181); these in turn have prescribed rules. He does not fully separate the fields of art and commerce but sees cultural production lying between two poles: autonomous and heteronomous. The autonomous pole involves art for art's sake with self-referencing values operating as an inversion of the profit motive in its seeking of symbolic capital (i.e., recognition). The heteronomous pole incorporates values both of cultural production and economics, with both cultural and commercial objectives. Bourdieu draws attention to the division between these poles of art and commerce - in the actors involved, the imperatives guiding them, their relations and the discourses among them. Competition lies within the fields and their institutions (Bourdieu, 1993b).

There have been a variety of interpretations of fashion though a Bourdieuian lens (e.g., Entwistle \& Rocamora, 2006; Hesmondhalgh, 2006; McRobbie, 1998). Although Bourdieu's major focus is on the field of art and he has comparatively little to say about the commercial field (Briggs, 2013; Hesmondhalgh, 2006), the commercial field is clearly also an elemental part of the fashion system. Either may represent a foundation for competitive differentiation and achievement in fashion. 


\subsubsection{Aspect 5: Design}

By design we include everything from individual inspiration to industrial production. It has been suggested that theorizing design has many problematic aspects (Eckert, 2001; Eckman \& Wagner, 1995). Those theories used in Fashion have tended to come from architecture and engineering (Au, Taylor, \& Newton, 2001; Watkins, 1988); and there are strong links to other design disciplines such as architecture and interior design - indeed in the U.S. there are cases where Fashion is paired with these in the same academic department.

Variations on the functional-expressive-aesthetic model have been widely used as the bases of discussions of fashion design (Lamb \& Kallal, 1992; Loschek, 2009) and there are a large number of facets of fashion design that might be considered as, at least in part, theoreticallybased such as composition, styling, color and symmetry (Brockman, 1965). However, broadly within design there has been little development of the theoretical foundations of how individuals respond to the aesthetics of design nor on its effect on consumer behavior (Veryzer, 2000). Fashion design is equally a process and a product and the extent to which one or the other aspect is guided by artistic inspiration and creativity, or commercial considerations is still being discussed (Borukhovich, 2009; Loschek, 2009; Mete, 2006).

Nonetheless, design and designers are situated at the heart of each of the four aspects. It is through design of apparel that creators and consumers of fashion interact. The choices a designer makes from fabrication to silhouette and textile choices can link the processes of production and consumption. Design translates and conflates the designer's vision with the commercial objectives of brands into the forms stimulating the cues and attitudes of consumers. It is through design that the aspects of art and commerce are actualized.

\subsection{The Fashion System}

A unified approach to the fashion system is needed to advance research and practice. Based on the propositions and counterpoints presented above, we developed a conceptual model of Fashion (Figure 2.6). The figure represents an integrated and comprehensive approach to Fashion - one that spans the wide range of aspects of the discipline, embracing the conceptual scope of the fashion system from the widest possible vantage point. These can lead to a more comprehensive exposition and understanding of the discipline. 


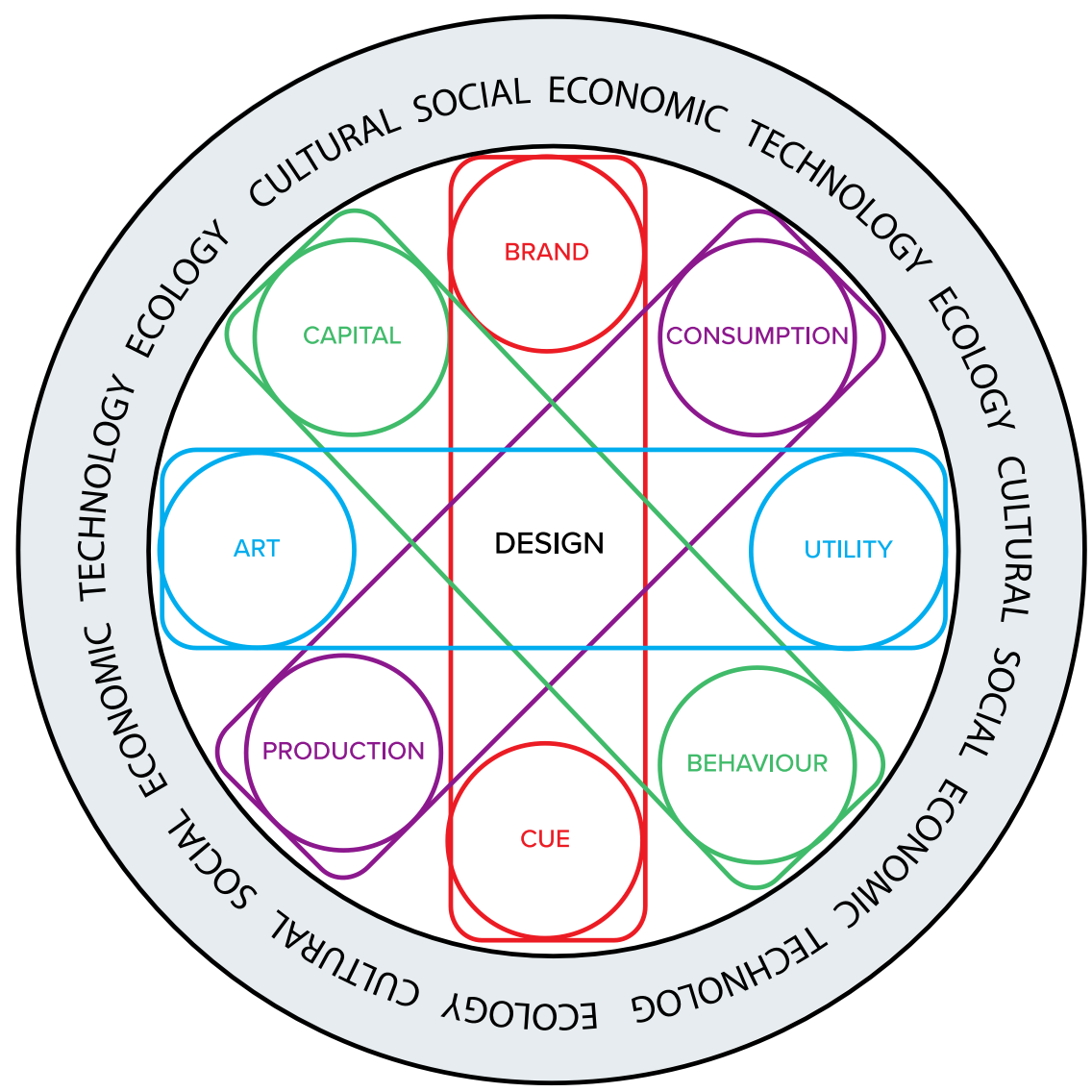

Figure 2.6: The Fashion System

Systems approaches seek to avoid reductionist viewpoints that focus on one or another aspect of the system — often reflecting a specialist's point of view. The whole is greater than the sum of the parts. This multi-dimensioned conceptual model of the system reflects the diversity of research interests and applications in the discipline. Figure 2.6 represents fashion as incorporating:

- Different perspectives (dimensions) on the nature of the system;

- Different units of analysis, layers of context, foci, and interactions;

- Different scales of interest

- The need for translation among the dimensions

It is crucial to recognize the importance of different scales of analysis. This will be demonstrated in examples below.

We present the system as a circle; metaphorically, there is no single entry point and no final end point. The core of the system is a conflation of the four aspects: agency, supply chain, 
merchandising, and motivation. These may be thought of as dimensions signifying the contributions of different disciplinary perspectives and theoretical underpinnings within Fashion. Each of these serves as a template to accommodate the core areas of research interest. The system is represented as a compendium of interfaces - a nexus of the counterpoints. Designs (and the designer) form the focus of each aspect (and the discipline as a whole).

The fashion system is imbedded within broader social, cultural, technological, ecological and economic systems. Fashion remains more or less permeable to outside influences and is linked to changes in those systems. Indeed, boundaries exist among the dimensions and with the greater environment. These connections can elucidate the dynamism of fashion change through time.

We see the model as a conceptual tool for thinking about the theoretical bases and relationships existing in fashion. It can suggest the relationships which exist within fashion frameworks and provide a guide for analyses and to focus research questions, methodologies and approaches.

It is important to note that, necessarily, research undertakings in fashion generally limit their scope of interest. Fashion looks different depending on where one is sitting in the system-there are different frames of reference for each part of the system. Figure 2.6 illustrates the various aspects of research interest in Fashion. Different theoretical constructs, methodologies and combinations of dimensions are incorporated into different areas of research. The visualization of how each fits within the entire system is useful when representing the scope of the discipline. 


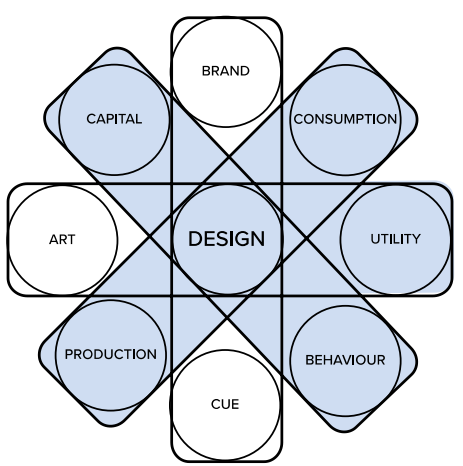

Fashion Sustainability

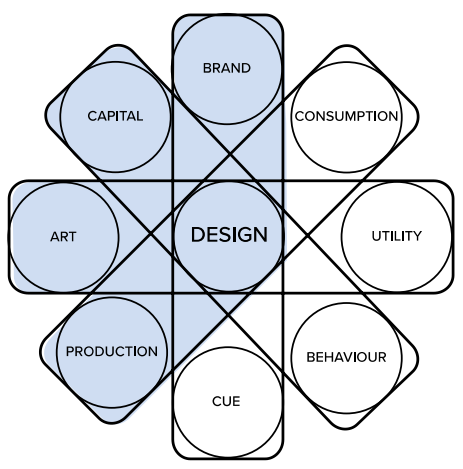

Fashion Production

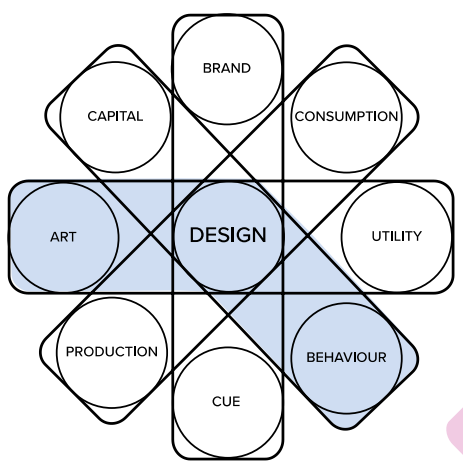

Fashion as Performance

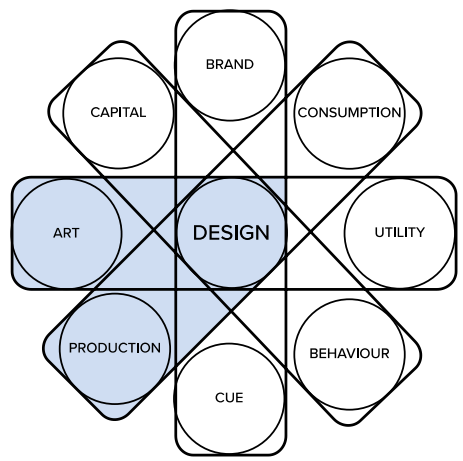

Fashion Style

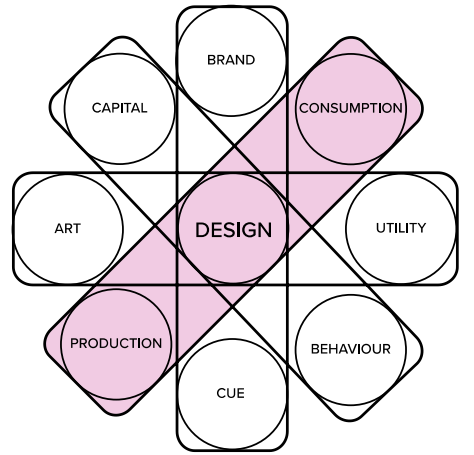

Supply Chain

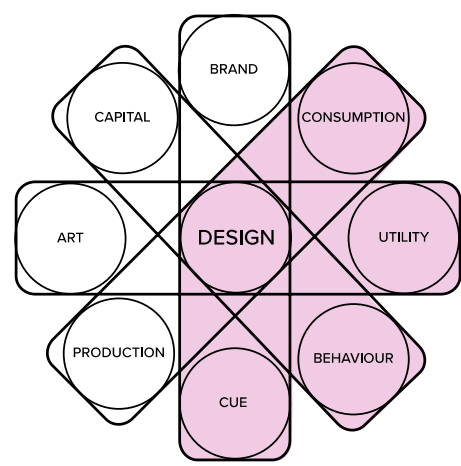

Consumer Behaviour

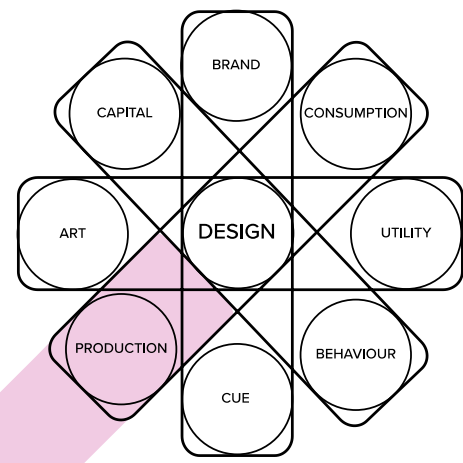

Textile Science

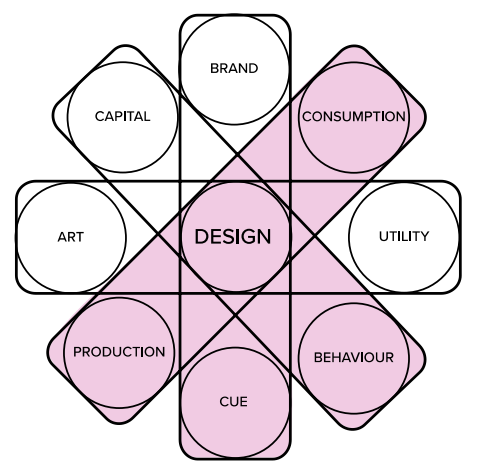

Fast Fashion

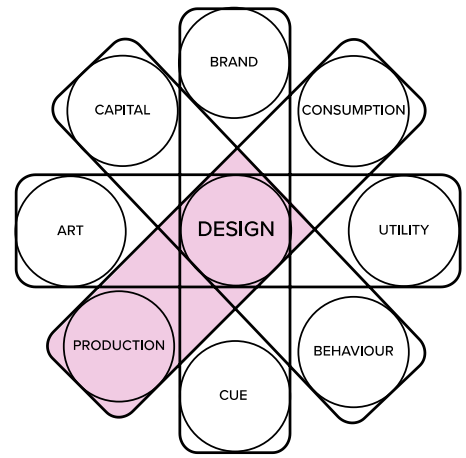

Design and Fabrication

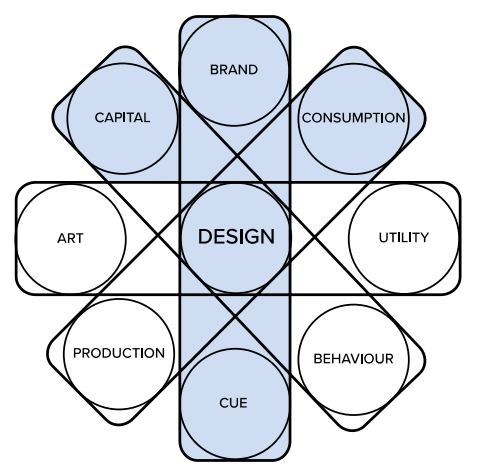

Fashion Merchandising

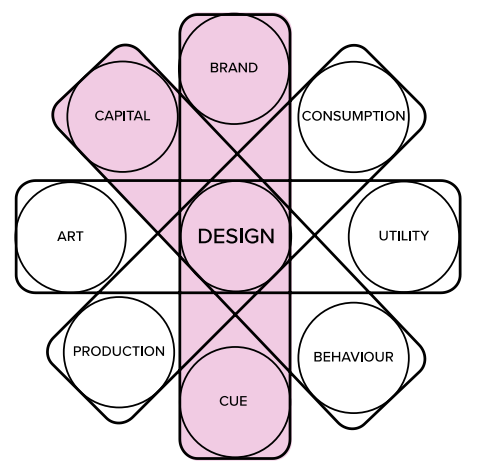

Fashion Branding

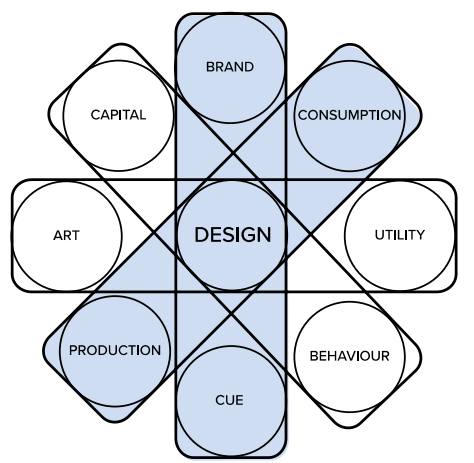

The Business of Fashion

Figure 2.7: Examples of Areas of Research Interest in Fashion 
Figure 1.7 contains areas of current research focus in Fashion. When we place many research interests together, we get a better sense of how all the various perspectives can be conceptualized. As a holistic framework, the model encourages one to consider where a research area sits within the scope of the aspects that make up the discipline of Fashion. The model reflects the diversity of research interests and applications in the discipline. For example, we see Fashion as Performance manifested through art, design and behavior. The main output of Fashion Branding, which involves capital, brand, design and cue, is to build symbolic capital and power relations (e.g. Vogue and editor-in-chief Anna Wintour). Fashion Merchandising builds upon Fashion Branding through the addition of consumption (e.g. retailing). While we see Textile Science to exist in production and in areas that live outside the Fashion System (e.g. chemical engineering and fiber and polymer science). The visualization, especially the outer ring which considers technological perspectives, nudges one to consider alternative theoretical lenses or applications.

Sustainable fashion should consider production, consumption, behaviours and cues. When seeking to understand why production and consumption have become so great, we must investigate the social processes driving desire and demand. For example, the advancement of communication technology has created platforms for everyone to share and participate in fashion. Greater participation in fashion through social media provides more cues to produce and consume fashion leading to new forms of social capital and a cult of celebrity within fashion (i.e. street style bloggers and fashion influencers). Therefore, the physical consumption of a fashion garment is concurrently the production of fashion culture. As long as these social pressures continue to increase (e.g. cues, democratization of fashion and photographic documentation via social media), so will our desire to produce and consume fashion in an unsustainable manner.

\subsection{Conclusion}

Generally, theories regarding fashion have been typically formulated from the vantage point of a specific area of focus - and these often derive from disciplines outside Fashion itself. These lenses often offer significant insight, but do not account for, the breadth of the multi-disciplinary nature of fashion. To describe this disparate character, this paper sources multiple theories from various disciplinary bases to provide an integrated construct of fashion. The single conceptualization seeks to represent and outline how the fashion system is currently constructed. Four distinct dimensions and counterpoints: (1) Agency: Creator - Consumer; (2) Supply Chain: Production - Consumption; (3) Merchandising: Brands - Cues; (4) Motivation: Art - Commerce, 
are merged into a conceptual unified theory of the fashion system. The central role of design and the designer is a foundational aspect of how we view fashion. This model encompasses the diversity of areas of theory and research interest in the discipline and counters reductionist viewpoints for understanding Fashion. The model introduces distinct units of understanding and analysis. This is essential as Fashion finds itself in a disruptive moment. The model fulfills the need for translation among the theoretical foundations which have been applied in Fashion. With a robust explanatory and heuristic value, the model may foster the creation of new concepts, theories and ideas that further the discourse and knowledge base of the discipline. 


\title{
3. TOOLS FOR SUSTAINABLE FASHION DESIGN: AN ANALYSIS OF THEIR FITNESS FOR PURPOSE
}

\begin{abstract}
Understanding the complexity of sustainable fashion issues can be overwhelming and a barrier for fashion designers. A number of tools for sustainable fashion design have been developed to aid designers in the integration of sustainability into their design practices. We analyze these to determine their fitness for purpose. Among them, three categories (archetypes) of tools are identified: Universal, Participatory, and Assessment. We propose an innovation framework and a five-dimensional model of sustainability specific to fashion to facilitate the analysis of the tools. Using the archetype categorization may facilitate designers in identifying the most appropriate type of tool for a specific circumstance, depending on context and need.
\end{abstract}

\subsection{Introduction}

There is widespread recognition that the fashion industry's current practices have adverse environmental, economic and social impacts (Fletcher, Grose, \& Hawken, 2012; Hethorn \& Ulasewicz, 2008; Karaosman, Morales-Alonso, \& Brun, 2016; Niinimäki \& Hassi, 2011). A growing body of literature, resources and tools supports the transition to sustainable practices. Fashion brands around the world are beginning to implement sustainability strategies and large companies, such as Kering, Zara, Nike, and H\&M, regularly issue publicly-available reports describing their sustainability activities. Likewise, many fashion micro- and small enterprises (MSEs) are adopting sustainable business practices. This is encouraging, as sectors all across the fashion industry from sportswear, footwear, to luxury labels and lingerie, need to develop sustainable products and services which consider environmental, economic, and social issues throughout their product life cycles and value chains (Allwood et al., 2008; Fletcher, 2008; Gwilt \& Rissanen, 2011; Hethorn \& Ulasewicz, 2008).

Designers are repeatedly declared as key agents of change in the transformation to a sustainable fashion industry (Fletcher, 2008; K Fletcher et al., 2012; Gwilt \& Rissanen, 2011; Niinimäki \& Hassi, 2011). Being proactive early in the design and manufacturing process is essential for meeting sustainability goals. The designer's role is expanding and should include 
devising courses of action, no longer avoiding the ethical questions associated with producing and consuming fashion (Krippendorff, 2006). Designers have the opportunity to increase sustainability in apparel design throughout every phase of the garment life cycle (Chapman, 2014; Fletcher et al., 2012). The need for designers to consider all phases throughout the design process in making sustainable fashion is a consistent theme in the literature.

To facilitate the shift towards sustainability, new concepts of design have emerged. Arising out of the ideas of "alternative design", "attitudinal design" and "design for need", eco-design became a key concept for those advocating for the socially and ecologically responsible design of products (Deaton \& Muellbauer, 1980; Knight \& Jenkins, 2009; Madge, 1993; Papanek, 1984). Eco-design is defined as "a design process that considers the environmental impact associated with a product throughout its entire life from raw materials through production and use to the end of its life" (Niinimäki, 2006, pg. 67). Subsequently, design for sustainability (DfS) was developed and as it has evolved, DfS has moved from having a technical product-centric focus to recognizing design as a vehicle for large scale systems level change (Ceschin \& Gaziulusoy, 2016). In response to the need generated by DfS, a variety of specific tools for sustainable fashion design ("Tools") have emerged to aid designers.

In order to identify existing tools, we have undertaken a thorough search of the literature and websites (institutions, organizations and NGOs). This resulted in the identification of twelve Tools (Table 3.1) each of which was developed specifically for use by fashion designers with the aim of improving and developing sustainable fashion. There is a wide variety of tools, some are developed by academic exercises while others are developed as industry partnerships, for example the Higg Index was based on Nike's Material Sustainability Index (Sustainable Apparel Coalition, 2018). Other tools exist that include textile materials such as the Idemat and Idemat Light app, a very comprehensive impact assessment tool that also includes eco-cost but does not fall within the criteria of this study (van der Velden, Patel, \& Vogtländer, 2014).

Unfortunately, the methods of the tools are not all described in a way that allows for a parallel organization of the tools, their development and methods. For example, addressing availability of the tools becomes difficult as availability changes over time and is dependent on the groups or individuals searching and accessing the tools. The complexity of dealing with all the variations of the tools is beyond the scope of this paper. 
Table 3.1: Tools for sustainable fashion design

\begin{tabular}{|c|c|}
\hline Tools & Description \\
\hline $\begin{array}{l}\text { Considerate Design (CDT) } \\
\text { (Black \& Eckert, 2012) }\end{array}$ & $\begin{array}{l}\text { A simple visual tool for designers to assess the environmental impact of a product or } \\
\text { particular design on eight dimensions: } 1 \text {. concept design, } 2 \text {. materials, } 3 \text {. } \\
\text { manufacturing, } 4 \text {. use phase/consumer, } 5 \text {. customization and personalization, } 6 \text {. } \\
\text { durability/longevity, } 7 \text {. transport, and } 8 \text {. end of use/disposal }\end{array}$ \\
\hline $\begin{array}{l}\text { Considered Take and Return } \\
\text { (CT\&R) (Aakko \& } \\
\text { Koskennurmi-Sivonen, 2013) }\end{array}$ & $\begin{array}{l}\text { Developed from a designer's point of view especially for small scale production. } \\
\text { The tool is based mainly on: } 1 \text {. Cradle to Cradle; } 2 \text {. Slow Fashion; and 3. Functional } \\
\text { Design. The model establishes a core category called "considered take and return" } \\
\text { for which all other categories are integrated. }\end{array}$ \\
\hline $\begin{array}{l}\text { Cradle to Cradle Apparel } \\
\text { Design (C2CAD) (Jin Gam, } \\
\text { Cao, Farr, \& Heine, 2009) }\end{array}$ & $\begin{array}{l}\text { The C2CAD model was developed to provide guidelines for apparel designers and } \\
\text { manufacturers by integrating the Cradle to Cradle model into two existing apparel } \\
\text { design and production models }[18,19] \text {. The model identifies four main steps where } \\
\text { designers and manufacturers should consider environmental impacts in their } \\
\text { decision making. }\end{array}$ \\
\hline $\begin{array}{l}\text { Cradle to Cradle Certified } \\
\text { Product Standard (C2C) (Cradle } \\
\text { to Cradle Products Innovation } \\
\text { Institute, 2018; Fashion for } \\
\text { Good, 2018) }\end{array}$ & $\begin{array}{l}\text { C2C, a not-for-profit organization, created a product standard framework for quality } \\
\text { assessment and innovation built upon circular thinking, a biomimetic approach of } \\
\text { design and science. C2C Certified created a Fashion Positive Materials Collection, a } \\
\text { digital resource of certified materials. The C2C Products Innovation Institute has } \\
\text { also created a sub organization entitled Fashion for Good offering a "How-To" } \\
\text { guide for implementing the C2C Certified Product Standard for garment } \\
\text { manufacturers. }\end{array}$ \\
\hline
\end{tabular}

Higg Index Material

Sustainability Index (HIMSI)

(Sustainable Apparel Coalition, 2018)

Higg Index Design and Development Tool. Rapid Design Module Beta Version (HI) (Sustainable Apparel Coalition, 2017)

MADE-BY (MB) (Made-By, 2018)

Nike Making App (NMA) (Centre for Sustainable Fashion, 2013; Nike Inc, 2015)

Sustainable Design Cards DSKD (SDC) (Design School Kolding, 2018b; Hasling \& Ræbild, 2017)

Sustainable Fashion Bridges Ideation Toolkit (SFB) (Hur, Beverley, \& Cassidy, 2013)
An assessment scoring tool that measures the environmental sustainability impacts of materials for use in apparel and footwear products. Originally developed by Nike in 2012, the MSI was adopted by Sustainable Apparel Coalition and integrated into the Higg Index. The Higg MSI is publicly available and intended to be used during a product's design phase to understand its predicted impact.

A product development and design tool that measures the environmental impacts of apparel, footwear and textile products. The RDM provides designers/developers with an internal facing score out of 100 points. The objective of the tool is to engage designers/developers to reduce impact.

A performance tracking tool created by Made-By, a not-for-profit organization, to support fashion brands and retailers improve their sustainability performance. The tool was designed in collaboration with industry professionals and sustainability experts. Verification and scoring are conducted by Made-By on a yearly basis.

A designer specific assessment tool that ranks the environmental impacts of materials used in apparel and footwear products. The objective is to provide a reference guide that compares impacts of materials in an accessible easy-to-use format. Materials are rated out of a score of 50 points. The Nike MSI provides the data for the model, an app available as a free download from iTunes.

A set of design cards developed to increase an apparel product's sustainability through longevity. The tool was created as part of a collaborative case study between Design School Kolding (DKSD) and Kopenhagen Fur, a local fur manufacturer. The cards are available as a free download and are intended for fashion designers.

This ideation toolkit is a design thinking tool intended to encourage designers to consider sustainability from the onset of the design process. The ideation tool 


\begin{tabular}{ll}
\hline Tools & Description \\
\hline & $\begin{array}{l}\text { includes a set of } 60 \text { cards using a co-design approach intended to be used by fashion } \\
\text { designers and highly engaged users during the ideation phase of the design process. }\end{array}$ \\
\hline $\begin{array}{l}\text { Sustainable Fashion Design } \\
\text { Model (SFD) (Gwilt, 2015) }\end{array}$ & $\begin{array}{l}\text { A model with the aim to support designers in the integration of sustainability } \\
\text { strategies within the design process. The tool requires designers to identify negative } \\
\text { impacts and employ appropriate strategies to reduce or eliminate the impact. } \\
\text { Developed over 5 years using empiric interviews, examination of garments and } \\
\text { practice-led inquiry and is presented from a life cycle perspective. }\end{array}$ \\
\hline TED's TEN (Textile & $\begin{array}{l}\text { A practice-based sustainable design tool developed by Textiles Environment } \\
\text { Design, a research centre based out of the University of Arts London. The design } \\
\text { cards are intended to assist designers in creating textiles and apparel that reduce the } \\
\text { environmental impacts of fashion products through a layered design thinking } \\
\text { approach. }\end{array}$ \\
\hline
\end{tabular}

The aim of this paper is to explore the existing sustainable fashion design tools, and through a comparative analysis, establish their fitness for purpose - that is, the ability of the tools to support and enhance sustainable fashion practice. This notion of fitness for purpose builds on Harvey and Green (1993, pg. 17), who state that fitness for purpose "identifies quality in terms of the extent to which a product or service meets the specifications of the customer." To date, no one has undertaken such an analysis of the range of Tools.

Little literature exists regarding the use of sustainable design tools in the fashion industry. Connor-Crabb (2017) examined three tools for sustainable fashion design through interviews with the tool developers to determine barriers and opportunities for tool use. The majority of the literature discusses the development of a particular tool, predominately through the description of case studies (Aakko \& Koskennurmi-Sivonen, 2013; Connor-Crabb, 2017; Gilbert, 2017; Gwilt, 2015; Hasling \& Ræbild, 2017; Jin Gam et al., 2009; Ræbild \& Hasling, 2018; Williams, 2013). Other studies have identified barriers for designers adopting these tools for sustainable design. A key barrier identified in the limited literature is awareness. Fashion designers are not aware of the existence of these tools nor are they using them (Connor-Crabb, 2017; Anika Kozlowski et al., 2018). Even among designers who are aware of sustainable design tools, inadequate knowledge regarding sustainability and lack of time contribute to an inability to effectively select and adapt tools to their practice (Bovea \& Pérez-Belis, 2012; Knight \& Jenkins, 2009).

Prior studies have also noted that learning mechanisms required to use a tool need to complement the way designers work (Bovea \& Pérez-Belis, 2012; Ceschin \& Gaziulusoy, 2016; Connor-Crabb, 2017; Lofthouse, 2006). Information needs to be presented in a style that is 
suitable for designers (Connor-Crabb, 2017; Lofthouse, 2006). Recently, Hur and Cassidy (2019) have conducted interviews with fashion designers to identify impediments in implementing sustainability - barriers identified include the lack of design-led approaches for implementing sustainability and perceived trade-offs with other design criteria such as aesthetic styles, costs, and fashion trends. Palomo-Levinski and Hahn's (Palomo-Lovinski \& Hahn, 2014) survey of fashion designers identifies that new approaches and a shift to sustainability in education are needed. There is a scarcity of education and understanding of sustainability in fashion, what real innovation for sustainable fashion should become and tangible ways to enact change in creating clothing. They found 86 percent of respondents agreed "that is was the responsibility of the designer to initiate change" (Palomo-Lovinski \& Hahn, 2014, pg. 94).

Learning and use can also become prohibitive as it becomes too time consuming to be carried out on a regular basis (Connor-Crabb, 2017; Lofthouse, 2006). This is especially relevant in light of designers citing the lack of consensus and knowledge regarding sustainable fashion and sustainable fashion design as a barrier to its wide scale adoption (Bovea \& Pérez-Belis, 2012; Anika Kozlowski et al., 2018). The complexity of sustainability has made it challenging for designers to implement appropriate solutions (Bovea \& Pérez-Belis, 2012; Connor-Crabb, 2017; Lofthouse, 2006) - leading to designers not using or integrating these tools or strategies in their daily design practices (Bovea \& Pérez-Belis, 2012; Lofthouse, 2006). This situation highlights an interesting predicament as solutions for sustainability frequently identify designers as key agents of change.

\subsection{Analytical Framework}

Solutions for sustainability frequently identify innovation, design thinking, systems thinking, and the use of a holistic perspective. Designers are seen as the nexus of technological integration, implementation, decision-making, creativity and novelty. Sustainable design includes understanding of the many complex and interacting issues that must be taken into consideration (Ceschin \& Gaziulusoy, 2016). Understanding the complexity of sustainable fashion issues can be overwhelming and a barrier for fashion designers, particularly those new to sustainable design (Bovea \& Pérez-Belis, 2012). As suggested by Ceschin and Gaziulusoy (2016) to obtain a real and lasting impact, there needs to be a greater understanding of the complexity and interacting issues within sustainable design. 
This need suggests approaches set forth by designers should aim to incorporate innovation in all aspects of their practice, vision and goals over time, but also to start simply and build complexity over time. Currently, tools for sustainable design fail to fully take the complex idea of sustainability and simplify it into clear digestible resources and actions that provide the foundation for accommodating increasing complexity over time.

Our analytical approach adopted in this paper incorporates three perspectives: innovation levels, dimensions of sustainability, and the interlinking of these as they effect sustainability in the fashion system. The analytical framework was developed based on a review of the literature and the tools reviewed in this paper.

\subsubsection{Innovation Levels}

Sustainable fashion can be achieved by building incrementally from an initial focus on product-level innovation through to more complex system-orientated levels of innovation. Strategies can be differentiated by the level or degree of innovation. The seminal paper on ecodesign by Brezet (1997) defines four levels of innovation: 1. product-level improvement, 2. product redesign, 3. function innovation, and 4. system innovation. As Brezet notes, there is increasing innovational freedom in eco-design types as you move from product- to system-level innovation. Similarly, Ceschin and Gaziulusoy (2016) identify four levels of innovation within DfS that expand in complexity and innovational freedom from product-level innovation to sociotechnical system innovation. In their approaches, innovation stops short of considering the nature of the linkages in the fashion system.

Despite a general recognition in the literature of the need to do so, the frameworks proposed by Brezet (1997) and Ceschin and Gaziulusoy (2016) do not incorporate elements of the system such as resiliency, adaptability and circularity. In addition, sustainability is not static and must continuously evolve within a set of critical limits (Mentz, 2012). The fashion industry must therefore also be understood as a system with a set of critical limits and a natural sense of disorder. Core considerations such as resiliency and adaptability can ensure the fashion system evolves within the identified set of critical limits. Transitioning to circular economies and closed-loop systems is not a static process nor a utopian ideal but rather a system that should adapt and be resilient. A new level of resilient, adaptable and circular (RAC) innovations, introduced in this paper, incorporates these concepts. 


\subsubsection{Dimensions of Sustainability}

Traditionally models of sustainability include three dimensions: 1 . environmental, 2. social, and 3. economic. However, for sustainable fashion design, these can be expanded. Our approach includes a five-dimension model for sustainable fashion by adding 4. aesthetic, and 5 . cultural dimensions (Figure 3.1). The dimension of aesthetic sustainability is reflected at the level of the product while cultural sustainability requires a systems-level approach.

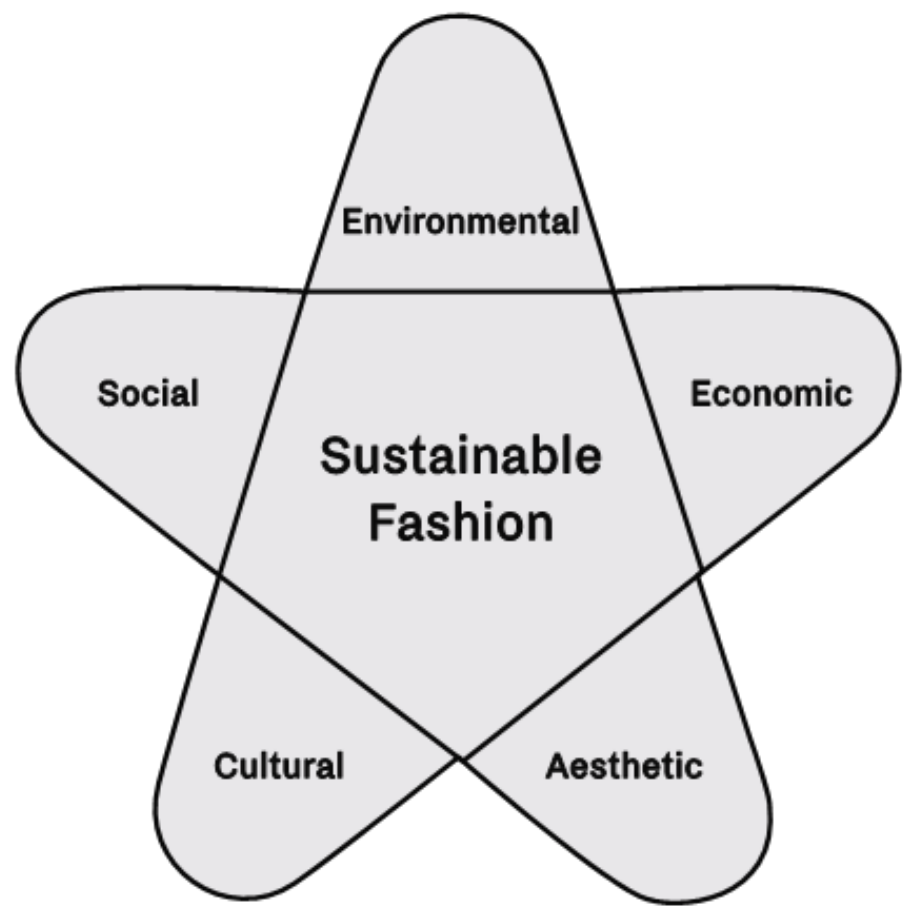

Figure 3.1 Five dimensions of sustainable fashion

Harper (2018) refers to aesthetic sustainability as timelessness, durability and continuous attraction to an object such as a garment. The work of Chapman $(2005,2014)$ and the recognition for the need for slow fashion by Fletcher (2010) demonstrates that aesthetic sustainability is a central consideration to sustainable fashion. Designing for sustainability in fashion must consider the sustainability of an individual's aesthetic experience (Flood Heaton \& McDonagh, 2017; Zafarmand, Sugiyama, \& Watanabe, 2003). Our approach in this paper incorporates aesthetic sustainability as an additional dimension.

The cultural dimension of sustainability derives from the notion that concepts of sustainability generally fail to differentiate among cultural systems, value systems, norms, behaviors and ideas and does not recognize the differences in local economies and ecologies. 
Cultural sustainability involves a shifting of beyond the consideration of social ethics to an acknowledgement of the exploitation of labor and resources and the legacy of colonization. Essingler (2011) notes designers should also consider cultural colonialism as industries develop new paradigms of sustainability and sustainable design. This promotes a more holistic perspectives when designers consider the cultural context for sustainable production and consumption. Modern Western culture has a tendency to focus on piecemeal strategies and/or encourages consumption of sustainable products at a higher price point. The Western solution is therefore often focused on recycling rather than reuse and reduction. Recycling does not seek to discourage consumption but maintains the idea that current consumption rates are okay as long as a recycling system is in place. Within Indigenous cultures, sustainability is inherent to their way of life [50]. The fashion industry should consider the inequities throughout the supply chain as the majority of apparel manufacturing occurs in developing countries. These are also the people and communities that can be negatively impacted by the fashion industry. This is an area of sustainable fashion that is yet to be addressed in the academic literature but has entered the sustainable fashion discourse by academics and practitioners within fashion.

The slow fashion movement advocates for clothing longevity and slowing down of the fashion system by building slow culture through a lens of systems thinking; key to this is recognizing that a sustainable fashion system needs a shift in culture (Fletcher, 2010; Štefko \& Steffek, 2018). To transform fashion, collectively, its cultural sustainability must be considered and addressed (Hur et al., 2013; Mora, Rocamora, \& Volonté, 2014). There is an industry culture that exists within fashion which is reflected through industry norms and values. Cultural sustainability recognizes the processes and the intrinsic qualities and values embedded in a garment.

\subsubsection{Interlinkages}

The levels of innovation and the dimensions of sustainability are interlinked and reflect the degree of potential impact of sustainability strategies on fashion. Three levels of impact were recognized as they relate to fashion: 1. "Improvement", 2. "Challenge" and 3. "Transformative". Impact refers to the level of which the tool is designed to affect the fashion system. Improvement occurs at the level of the product and tends to focus primarily on environmental impacts.

Challenge addresses change in aspects of the existing system and incorporates four dimensions of sustainability: environmental, social, economic and aesthetic. Transformation changes the 
fundamental nature and behaviors of a system which requires the holistic inclusion of the five dimensions of sustainability.

This is not to say that strategies for product-level innovation are any less effective than those that are transformative. For example, if all fashion businesses switched to using only organic cotton, collectively this would have a great impact, but not a transformational one. An organic cotton product would still exist in an unsustainable system that does not consider additional processing, use or disposal. Transformative impact would be, for example, the use of organic cotton in a garment produced ethically and embedded in a circular closed-loop system that considers all phases of a garment lifecycle from raw materials to end-of-use, and local ecologies, cultures, social systems and economies (Hu, Li, Chen, \& Wang, 2014). Infrastructure and social behaviors and norms would support the ability for circular closed-loop systems to exist, ensuring garments remain in regenerative loops.

To determine the fitness for purpose of the Tools, four objectives were developed: 1 . to identify existing tools for sustainable fashion design; 2 . to create a systematic approach to identify their character and utility; 3. within each tool, to identify the individual strategies for sustainable fashion design ("Strategies"); and 4) to undertake a critical analysis of the value of the tools as a means for sustainable fashion design. We created a sustainable fashion innovation framework to facilitate the categorization of the Strategies (Table 3.2).

Table 3.2: Sustainable fashion Innovation framework.

\begin{tabular}{|c|c|c|c|c|c|}
\hline \multirow{2}{*}{\multicolumn{2}{|c|}{$\begin{array}{l}\text { Impact } \\
\square \text { Improvement } \\
\square \text { Challenge } \\
\text { Transformative }\end{array}$}} & \multicolumn{4}{|c|}{ Level of Innovation } \\
\hline & & \multirow{2}{*}{ Product } & \multirow{2}{*}{$\begin{array}{l}\text { Product- } \\
\text { service social } \\
\text { system }\end{array}$} & \multirow{2}{*}{ System } & $\begin{array}{c}\text { Resilient, } \\
\text { adaptable } \\
\text { and circular } \\
\text { (RAC) } \\
\text { innovation }\end{array}$ \\
\hline \multirow{5}{*}{$\begin{array}{l}\text { Dimensions } \\
\text { of } \\
\text { Sustainability }\end{array}$} & Environmental & & & & \\
\hline & Social & & & & \\
\hline & Economic & & & & \\
\hline & Aesthetic & & & & \\
\hline & Cultural & & & & \\
\hline
\end{tabular}




\subsubsection{Improvement}

The Improvement category addresses impacts that operate solely at the level of the product. Strategies that are categorized as Improvement are product-level innovations which seek to improve existing and/or develop novel products (Ceschin \& Gaziulusoy, 2016). Most seek incremental improvements that are low hanging fruit. While they may also target social dimensions of sustainability, product-level innovations are largely environmentally-driven, insular, and short-term (Brezet, 1997; Ceschin \& Gaziulusoy, 2016). They do not incorporate economic or financial considerations such as new sustainable business models. Approaches, strategies and innovations at the level of the product generally involve one or more of: 1. product improvement, 2. production process improvement, and 3. product redesign. Examples of Strategies categorized within this level include those in which the environmental consideration is the basis for opting for renewable energy sources, designing for reuse, implementing zero waste pattern drafting, and sourcing environmentally friendly or low impact materials.

\subsubsection{Challenge}

Strategies classified as Challenge are those which focus on impacts that target individual behavioural change. This requires a more holistic perspective and the use of systems thinking. Applying systems thinking within the fashion context entails a broader view of sustainability, one that incorporates four dimensions: environmental, social, economic and aesthetic. Design approaches need to live in new conceptual spaces that consider many more aspects such as the temporality, processes, inputs and outputs of production and consumption of products and services within broader social contexts; essentially shifting to a holistic viewpoint of the fashion system. Systems thinking pushes designers to consider both how the industry is organized, and the resulting behaviors (individual and systems level). Understanding current behaviors opens up exploration into how behavior change principles can encourage people to act in ways that benefit the planet. Those Strategies which address impacts that Challenge the fashion industry have influence at two levels of innovation: 1. product-service social systems, and 2. systems innovation.

Innovations at the level of product-service social system begin to challenge current system norms and operations within the fashion industry. These include changes to the traditional business model in fashion (Thorisdottir \& Johannsdottir, 2019). Product-service systems move away from the traditional linear model that produces individual products towards integrated 
combinations of products and services. This typically involves the development of new business models with value propositions oriented to satisfy users through the delivery of services instead of products (Ceschin \& Gaziulusoy, 2016). Integrating sustainability strategies into the role of designers requires that they engage with the models of business (Balkenende \& Bakker, 2018) and adopt new sustainable models into existing, and for new fashion businesses (Kozlowski et al., 2018).

Drawing on Manzini's (2015) ideas of Design for Social Innovation and Ceschin and Gaziulusoy's (2016) spatio-social system innovation type, we propose the product-service social systems innovation for sustainable fashion: a sustainable design approach that integrates products and services that considers societal needs and social innovation at different scales, for example decisions from that of the individual designer to the fashion industry itself. Collaborative work and community building are central to orientating, implementing and sustaining processes of social change through design. This includes consideration of four dimensions of sustainability: environmental, social, economic and aesthetic. Examples of these Strategies categorized within this level include adopting co-design, services for longer or intense utilization, rental services, designing modularity into garments, open-source fashion platforms, and community learning opportunities.

Systems innovations are those which begin to tie together product-level innovation and sustainable business models to the larger existing operations and infrastructure of the industry. Brezet (1997) identifies two types of innovation that exist within a systems-orientated lens: 1. the conceptual definition of new products, and 2. the definition of new production systems. These types of innovation necessitate changes to infrastructure and organizations that are required to support products and services (Brezet, 1997). Innovations draw upon systemic design approaches, in which local production systems are designed with waste from one production process becoming input for other processes (Ceschin \& Gaziulusoy, 2016). Examples of Strategies categorized within this level include design activism, design for social innovation, rethinking durability or creating open-source networks.

\subsubsection{Transformative}

Transformative Strategies seek to "transform the way clothes are designed, sold, and used to break free from their increasingly disposable nature" (Ellen MacArthur Foundation, 2017.pg. 24 ) in order to achieve a sustainable fashion system. Transformation of this magnitude dictates 
culture change where social behaviors are the focus. Strategies categorized at this level focus on innovations that emphasize reducing consumption and increasing circularity, adaptability and resiliency within unstable systems. Additionally, the RAC innovation level considers all five dimensions: economic, environmental, social, aesthetic and cultural.

The cultural dimension is an area severely under-addressed within sustainable fashion. Incorporating the cultural dimension ensures we do not propose the same blanket solutions globally and consider local systems. Supporting a sustainable fashion system in France could look dramatically different than what would work in Brazil, Bangladesh or Australia. This includes considerations such as local culture, political climate, economy and infrastructure. A cultural dimension also includes issues around postcolonialism and diversity. Sustainability issues such as climate change and biodiversity loss disproportionately harm the poorest people the very people who often manufacture fashion clothing (Minney, 2017). To ensure all stakeholders and voices along the fashion value chain are part of the solution, collaboration and transparency are vital for transformative change that includes new cultural narratives around sustainability.

The fashion industry has become incredibly complex, (Ellen MacArthur Foundation, 2017; Hur \& Cassidy, 2019) unpredictable and subject to disruption. There is a need therefore to recognize that there is a natural sense of disorder in existing systems, rather than an idealized system that rests in a harmonious balance (Mentz, 2012). Thus, in the transition to circular economies and closed-loop systems, resiliency and adaptability must be a core consideration.

Circularity is key component of transformative change. Redesigning of a linear system towards a circular economy with material regeneration at its core that includes local and global scales and alignment with the various speed cycles that exist in fashion (fast vs. slow fashion). Earley (2017) discusses sustainable design strategies and the importance of considering speeds within the context of circular fashion. Circularity requires both that lifecycle phases of a garment be considered at the design stage and that the original design needs to consider a multitude of lifecycles within different speed cycles (Earley, 2017). Circular economies are based on principles of closed loops and are restorative and regenerative by nature. Natural systems are regenerated, and economic growth is decoupled from consumption of finite resources (Ellen MacArthur Foundation, 2019). Design for circular economies considers certain core values which must be embedded into the design process. Principles such as ensuring "clothes, fabric, 
and fibres are kept at their highest value during use, and re-enter the economy after use, never ending up as waste" (Ellen MacArthur Foundation, 2017, pg. 22).

Design is therefore used to understand and solve complex problems, building upon the various levels of innovation and Strategies. Perspectives are broad and holistic and focus on developing new cultural norms and narratives that support sustainability with a positive impact on the future well-being of earth and its living systems. The goal is radical change that support ecological, economic, social, cultural and aesthetic needs by exploring synergies of multiple approaches, methods and strategies through a lens of systems thinking. Exploring synergies of multiple approaches requires foresight, meaning research and development time is crucial to creating these circular systems, as is openness to new technologies such as artificial intelligence. Artificial Intelligence is crucial to circular economies as it has the capabilities to support faster and more agile learning processes with iterative cycles of designing, prototyping and gathering feedback. This is necessary in a transition to an economy focused on keeping products and materials in use (Ellen MacArthur Foundation, 2019). Designers must embrace their new role which requires a more multi-faceted, collaborative and transparent approach. Examples of Strategies include designing for different speed systems, working within geographical fibresheds (i.e. working with plants, animals, materials and fibres found within the local geography), developing localization and addressing power shifts.

\subsection{Analysis}

The framework for the analysis was derived from a close examination of the tools. It was clear that the tools incorporated a wide variety of different sustainability strategies. The strategies were initially coded and categorized by the phases of a garment lifecycle which the tools incorporate. The strategies were also coded based on the five dimensions of sustainability. Based on this initial coding process, it became evident the potential impacts of the strategies differed (i.e. improvement, challenge and transformative).

The analysis of the tools was conducted through the development of: 1. a heatmap, 2. a parallel coordinate plot, and 3. a cluster analysis. Each of these provides a means for the visualization of the nature and relationships among the tools. Initially, the attributes and strategies were identified: 
1. The basic nature (the "attributes") of the tool (i.e., approach, scope, basic structure, purpose, application and character) as described in the instructions, descriptions, reviews and case studies;

2. The strategies (i.e., design types, methods, initiatives, strategies, approaches, and examples).

Categorization of the attributes and strategies was done inductively (Hsieh \& Shannon, 2005).

\subsubsection{Heatmap Development}

Heatmaps have been widely used in the literature to represent row and column hierarchical cluster structures of data in a rectangular colour-shaded matrix display. The matrix is a series of tiles represented by a shade from a colour scale to denote a value of the corresponding element of the data. The heatmap is typically used in the natural sciences to display large, complex data sets with hierarchical cluster trees appended to its margins (Wilkinson \& Friendly, 2009). For the purposes of this paper, we have used a simplified version of a heatmap as our dataset is considerably smaller than the typical datasets used within the natural sciences for bioinformatics display.

Among the twelve Tools, 520 Strategies were identified (Table 3.3). Among these, 367 distinct Strategies were identified of which $324(88 \%)$ were classified as product-level innovation, 21 (6\%) were product-service social innovation (PSSS), 17 (5\%) were system innovation and $5(1 \%)$ involve resilient, adaptable and circular (RAC) innovation. At the product-level, $70 \%$ of strategies at this level are unique to a specific tool and not used by any other tool. Among Strategies categorized at product-level innovations, $80 \%$ are solely focused on environmental impact. 
Table 3.3: Categorization of SSFD by impact and innovation level

\begin{tabular}{llllll}
\hline & \multicolumn{3}{l}{ Impact and Innovation Level } & \\
& \multicolumn{1}{l}{ Improvement } & Challenge & Transformative & Total \\
\cline { 2 - 5 } & Product & PSSS & System & RAC & 367 \\
\hline Count of unique SSFD & 324 & 21 & 17 & 5 & 520 \\
\hline Total count of SSFD & 466 & 28 & 18 & 8 & 397 \\
\hline $\begin{array}{l}\text { Count of } \\
\text { environmentally focused }\end{array}$ & 375 & 14 & 8 & 0 & \\
SSFD & & & & & \\
\hline
\end{tabular}

PSSS: product-service social innovation; RAC: resilient, adaptable and circular innovation

A heatmap (Figure 3.2) was created which highlights the distribution of the Strategies categorized by innovation levels and system impact. The heatmap indicates the number of Strategies classified at the levels of innovation and impact on fashion for each Tools. Each tool includes a focus on product-level innovation; five are solely designed to do so. Only three tools, CT\&R, SFB and Ted's Ten, incorporate Transformative consideration. The others consider some elements of PSSS, System or RAC, but these Strategies are few in number. The heatmap highlights the interlinking of the innovation levels, potential impact and strategies used. The relationship among strategies, innovation levels and potential impact becomes visible, demonstrating a difference among the various tools. 


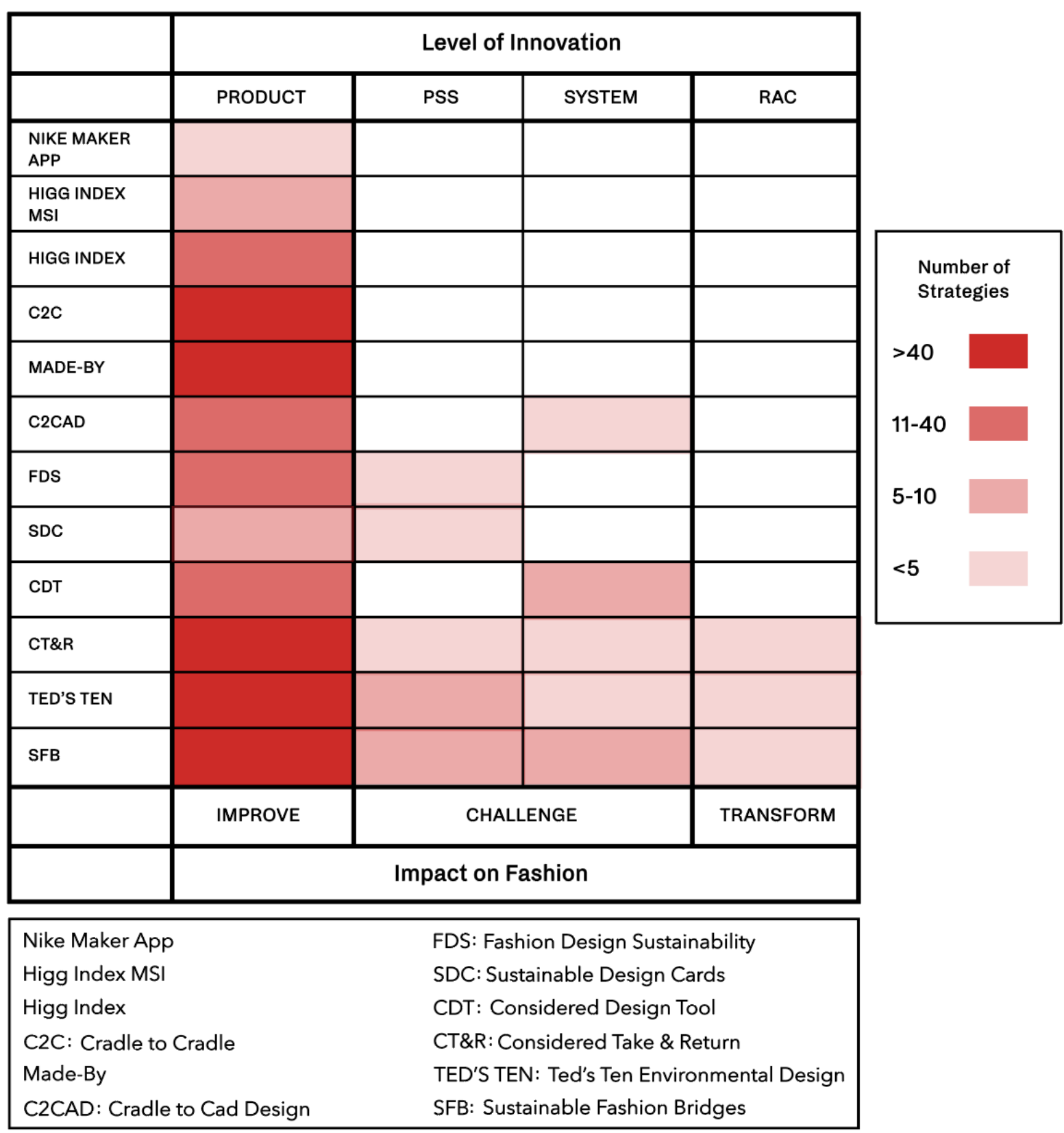

Figure 3.2: Heatmap of strategies for sustainable fashion design

\subsubsection{Parallel Coordinate Plot}

To better appreciate the overall nature of the tools, a parallel coordinate plot (PCP), a useful tool for visually examining multivariate data, was developed. This allows for comparison of multiple design tools among many design dimensions and variables (Pousman \& Stasko, 2006; Wölfel \& Merritt, 2013). Pousman and Stasko (2006) and Wölfel and Merritt (2013) have used PCPs to better understand design frameworks by comparing their attributes along various design dimensions. A major advantage of PCP is the capacity for comparison of multiple 
variables within an identified design dimension (e.g. the scope and the purpose of tool). The aim of applying this method is to determine whether any patterns exist.

To appreciate the benefits of current sustainable design tools, we conducted a survey of their attributes. Initially, four out of the five design dimensions from the Wölfel and Merritt (2013) study were deemed to be appropriate: 1. Purpose and Scope, 2. Duration, 3. System, and 4. Formal Qualities. For this study, five additional attributes were identified: 5. Categorization, 6. Accessibility, 7. Format, 8. Scoring/Assessment, and 9. Sustainability Dimensions. These nine design dimensions form an axis, which allows for a visual comparison when tool attributes are plotted along the dimensions, creating the PCP. The axes reflect the unique characteristics of sustainable fashion design identified throughout the coding process. Plotting of the attributes of the twelve Tools across the nine design dimensions allow for any patterns to become apparent. Patterns highlight similarities and differences among the Tools. The Tools are grouped based on similarities and differences to distinguish and identify any archetypes. Graduations within the design dimensions are used to differentiate the Tools (Wölfel \& Merritt, 2013). Descriptions of the nine design dimensions and the graduations within each are listed in Table 3.4.

Table 3.4: Descriptions of the nine design dimensions of the parallel coordinate plot (PCP)

\begin{tabular}{|c|c|}
\hline $\begin{array}{l}\text { Design } \\
\text { Dimensions }\end{array}$ & Description \\
\hline Categorization & $\begin{array}{l}\text { Three types of frameworks are used to organize information contained within the Tools. All } \\
\text { of the tools identified and positioned themselves within certain types of frameworks, } \\
\text { guiding the underlying philosophies of the tools: } 1 \text {. design types, } 2 \text {. apparel design model } \\
\text { thinking/design thinking, and 3. life cycle phases of garments. }\end{array}$ \\
\hline Purpose and scope & $\begin{array}{l}\text { look at the goal or aim of the tool and whether the Tools focus on a particular context. Three } \\
\text { graduations were used as identified by Wölfel \& Merritt [60]): 1. general, 2. participatory } \\
\text { design, and 3. context specific/agenda-driven. }\end{array}$ \\
\hline Duration & $\begin{array}{l}\text { Duration looks at the length of use of the Tools throughout the design process and whether } \\
\text { they are to be used at specific points within the process. This dimension addresses key } \\
\text { differences in length of time and point of time the Tool is to be used: } 1 \text {. anywhere/anytime, } \\
\text { 2. as needed/beginning of, and 3. specific point. }\end{array}$ \\
\hline System & $\begin{array}{l}\text { refers to methodology of use, as some Tools are used quite freely with no suggested } \\
\text { structure while others provide specific instructions on use [60]. Tools are created with an } \\
\text { intended approach and use by designers or a system of use. Three types were found among } \\
\text { the tools: } 1 \text {. no system, } 2 \text {. suggestions for use, and 3. specific instruction. }\end{array}$ \\
\hline Accessibility & $\begin{array}{l}\text { Accessibility is a major factor in a designer's ability to use existing tools. Three main } \\
\text { categories were found within this dimension: } 1 \text {. buy tool/journal article, } 2 \text {. free digital } \\
\text { download/access, and 3. paid membership required. }\end{array}$ \\
\hline Format & $\begin{array}{l}\text { Tools are classified by their format for use. How a designer interacts with tools is tied to } \\
\text { their format such as physical cards, inputs through an online platform or a two-dimensional } \\
\text { model drawing. Four formats were found among the tools: } 1 \text {. cards, } 2 \text {. models, } 3 \text {. standards } \\
\text { and } 4 \text {. impact assessment. }\end{array}$ \\
\hline
\end{tabular}




\begin{tabular}{ll}
\hline $\begin{array}{l}\text { Design } \\
\text { Dimensions }\end{array}$ & Description \\
\hline Formal qualities & $\begin{array}{l}\text { Formal qualities look at how the tool is organized for use. Three categories represent the } \\
\text { range of design tools: 1. categories, 2. only text/models, and 3. online } \\
\text { platforms/components. }\end{array}$ \\
\hline $\begin{array}{l}\text { Scoring/ } \\
\text { assessment }\end{array}$ & $\begin{array}{l}\text { Scoring/assessment determines the type and level of assessment employed by design tools. } \\
\text { The tools were found to have either: 1. no assessment, 2. levels, and 3. metrics (quantifiable } \\
\text { scores). }\end{array}$ \\
\hline $\begin{array}{l}\text { Sustainability } \\
\text { dimensions }\end{array}$ & $\begin{array}{l}\text { This represents the various dimensions of sustainability addressed by the tools. Three } \\
\text { variations of sustainability dimensions were found among the tools: } 1 \text {. Environmental, social } \\
\text { and aesthetic, 2. Environmental and social and 3. Environmental. Economic sustainability } \\
\text { was not prevalent and frequently addressed as a secondary consideration under } \\
\text { environmental or social sustainability. }\end{array}$ \\
\hline
\end{tabular}

We identified archetypes by grouping those tools that are generally similar as Universal, Participatory, and Assessment (Figure 3.3). Three of the Tools are classified as Universal, two as Participatory and seven as Assessment. The tools classified in each of the archetypes share a common set of characteristics. Table 3.5 provides a summary of these. 


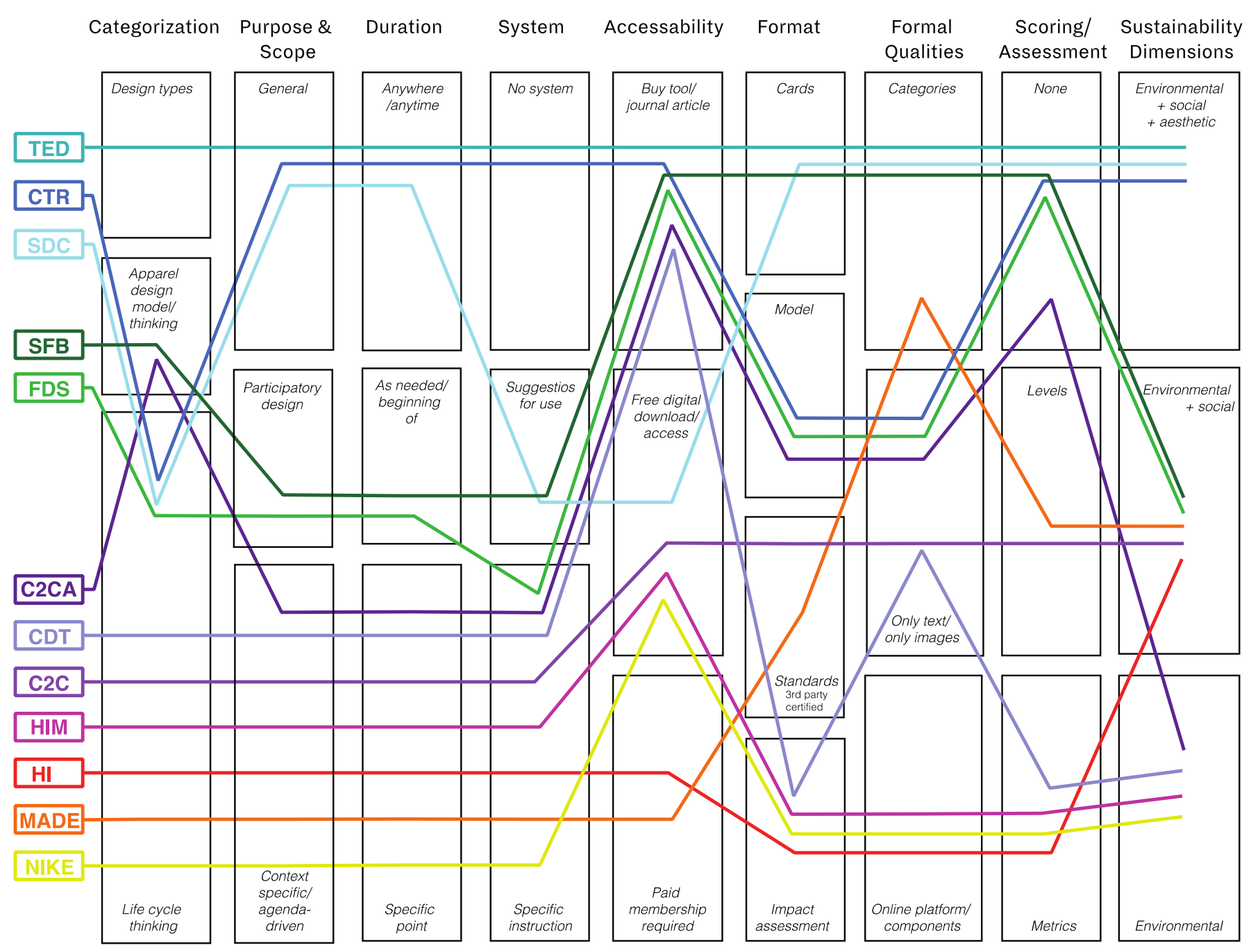

Figure 3.3: Parallel coordinate plot of the Tools.

Table 3.5: Tool Archetype descriptions.

Description
Distinguishing Characteristics
Sustainability Dimensions
- Do not have a specific duration or system for use.

- Can be easily applied and used within any part of the design process depending on Strategies selected

- Minimal to no instruction on their use as they are intended to be general

- Guidance comes in the form of examples and further readings, leaving further learning for the designer to seek out.

- Information is presented in an easy to use format such as visual two-dimensional models and/or physical cards
Environmental, social and aesthetic 
- The designer is central to this process,

Tools prescribe various

Participatory

- Sustainable Fashion

- Bridges Ideation Toolkit

- Sustainable Fashion Design Model approaches for co-design methods where designers build empathy and engage consumers in the design process. This builds sustainability through added- value by enhancing consumer experience and emotional durability of both product and process.
Assessment

- $\quad C 2 C$

- $C 2 C A D$

- Considered Design Tool

- Higg Index

- Higg Index MSI

- Made-By

- Nike Maker App
To identify and reduce negative environmental and social impacts of products and production processes. seeking change on a deeper level that is long term.

- Intended to be used at the beginning and then as needed throughout the design process, depending on how involved the consumer is in the co-design process.

- Instructions and context of use are more specific than Universal

- Design process is extended/more time consuming

- Information is presented in an easy to use format such as visual two-dimensional models and/or physical cards
Environmental and social

- Agenda driven and are context-specific

- Are used at specific points within the design process

- Categorized by life cycle phases

- Require specific instruction on use

- Utilize a scoring mechanism to assess impacts

- Require working with suppliers and factories to collect, monitor and improve upon environmental impacts

Largely Environmental with minimal attention to the social dimension

- Provide excellent additional support to tools categorized under Participatory and Universal

\subsubsection{Tools Classification}

To provide a holistic visual representation of the Tools we combined all the information into a single figure. We used a cluster analysis approach: a "cluster analysis" being generic name for a wide variety of procedures that can be used to create a classification (Guest \& McLellan, 2003). In this study, we used a heatmap and PCP to begin identifying groupings or clusters. By combining the results of the two approaches, we created a graphical representation for the categorization of the Tools, as shown in Figure 3.4. 


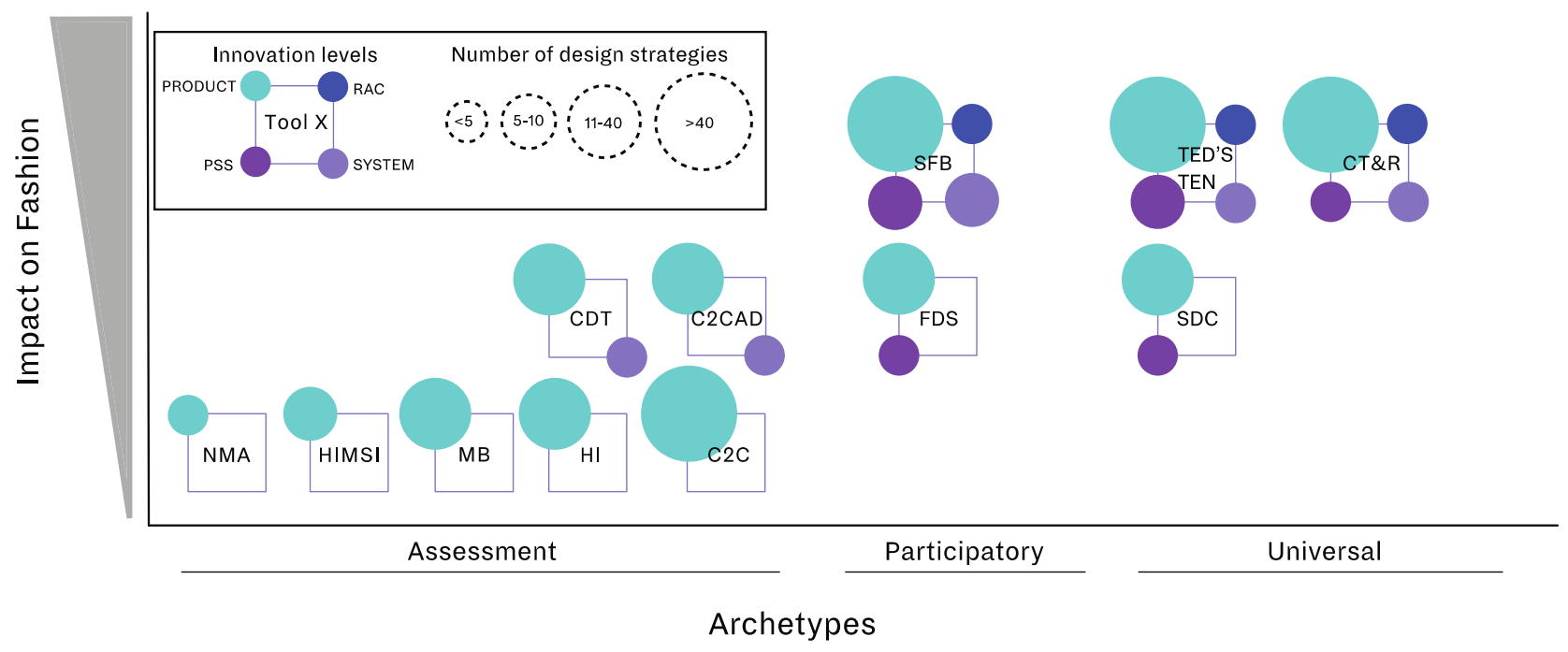

Figure 3.4: Classification of Tools

Figure 3.4 was constructed to group the three different archetypes. The vertical axis represents an increasing system impact of the archetypes based on levels of innovation - this reflects the impact on fashion. The circle size indicates the number of strategies within each archetype. For example, Ted's Ten includes 82 strategies at product-level innovation, 8 strategies at the PSSS level innovation, 3 strategies at the system-level innovation and 3 at the RAC level innovation. Classifying the Tools by level of innovation and count offers an impression of the potential system impact a designer could expect by using a particular Tool archetype. As a composite of the previous figures and analysis, Fig. 3.3 demonstrates that those tools categorized as Assessment are primarily focused on product level innovations. Tools such as the Higg Index prioritize strategies the reduce environmental impact of product and processes using LCA's and metric indicators to assess, monitor and improve identified impacts (e.g., reducing water usage or eliminating hazardous chemicals). Those tools that are more conceptual and seek to change how designer's work (e.g., behaviours and culture) and includes strategies that contextualize sustainability beyond the product. For example, Ted's Ten promotes design activism, a strategy that moves beyond products to consider designing events and communication strategies, targeting behaviours, lifestyle and culture change (Textile Environment Design, 2018). The designer assumes a role of a social innovator to activate positive societal changes for a sustainable fashion system. 


\subsection{Discussion}

This study offers the first comparative analysis of the Tools and an evaluation of their fitness for purpose for fashion design. Only one study has examined three tools through interviews with developers to determine barriers and opportunities for use (Connor-Crabb, 2017). The majority of studies into design tools typically document the development of a tool through a case study (e.g., Design School Kolding, 2018; Nike Inc, 2015). Essentially, the literature demonstrates much work is still needed as many barriers still exist for tool adoption and the integration of sustainability into design practices by designers for a variety of reasons.

To better understand the landscape of Tools within fashion, this study resulted in the creation of a Sustainable Fashion Innovation Framework and the identification of three tool archetypes: Assessment, Participatory and Universal. The Assessment tools differ significantly in the number of strategies they incorporate. However, they all primarily focus on the environmental dimension of sustainability - with social dimensions also considered in a minority of the tools. The purpose of these Tools is to aid designers throughout the design and product development process. They highlight the potential impacts a designer would encounter. These tools have been primarily developed through industry partnerships. The Participatory tools are characterized very specifically by engaging the consumer in the design process. These are specialized approaches that use co-design as their framework. The premise is that sustainability is enhanced when consumers are involved in the design and product development process as their perceptions and values towards garments are altered. These tools provide strategies to facilitate the concept. The Universal tools are inspirational in their character. They are a compendium of the various sustainable design methods, approaches, strategies, information sources and examples. They offer the greatest breadth of opportunity for integrating sustainability into the design process; however, they have limited to no instruction for use. These tools give the greatest freedom for designers to make choices about their design practice which may make them appealing.

Many of the Strategies identified overlap and are complementary. Solutions to sustainability do not exist in a vacuum, they must be holistic and be able to build upon each other as they increase in complexity. Tools should look to guide designers in how to frame the problem and consider alternative lenses when designing. These findings may help designers find new ways of combining Strategies and Tools that complement one another. Most designers begin working on sustainability in one area, like sourcing more sustainable materials, eliminating bad chemicals 
from products, or focusing on fair trade labor practices. While those efforts make a difference, sustainability does not start and stop with one initiative alone. Tools should be viewed within a landscape of tools where tools tackle different facets of the issues the fashion industry is facing; for example, Assessment tools pair well with those from the Universal or Participatory archetypes. Universal tools that suggest co-design methods could direct designers to Tools categorized as Participatory.

Key findings from this analysis include tool access and awareness, barriers for uptake, positive attributes and recommendations for tool development. Many barriers for tool uptake by practitioners were identified. One of the critical issues is tool accessibility. Tool access is problematic for several reasons. The majority of sustainable fashion designers are MSEs (Kozlowski et al., 2018); there is a financial barrier (especially for Assessment Tools) as membership, or consultation fees are quite expensive as they (e.g. Higg Index, Made-By, Cradleto-Cradle, $\mathrm{C} 2 \mathrm{CAD}$ ) have been developed by and for large corporate businesses. In addition, these tools typically require extensive expertise, time commitments and third-party auditing. As these Assessment Tools are intended for designer use, they are quite technical and require training or learning for use. In reality, they do not speak to designers, can be confusing and difficult to use as they have been found to be unappealing to fashion designers, cited as being too technical and scientific (Kozlowski et al., 2018).

Distinct from those designed for large corporations, each of the Universal and Participatory Tools was the result of a project-based academic initiative. These tools were developed for designers working in small companies. To provide access for fashion designers they have minimal to no cost. These tools experience barriers due to a lack of the funding that allows the tools to be updated and readily available; in addition, funding may not be available for tool testing, customizing, updating and promoting as these are dependent on time and resources within the scope of a project (Connor-Crabb, 2017). Certain tools are no longer available online or for purchase.

Tools that are available for free are difficult to find as they exist in different platforms and spaces such as websites, journals, academic books, apps or physical cards. This begs the question how designers with limited resources, time and access would successfully locate these tools. If designers are able to locate these tools, implementing tools independently at the very least requires an investment of time on behalf of the user (Connor-Crabb, 2017; Lofthouse, 2006), a difficult endeavor for MSE fashion designers. 
There is also no guidance for designers as to what tool is most appropriate for them. These tools tackle different facets of the sustainability issues within fashion and therefore have specific focuses and concomitant limitations. As suggested by Connor-Crabb (2017), successful implementation in practice is contingent on how clearly the application and limitations of a tool is described. What we found is certain tools are suited to be used together and inter-changeably. Tools currently exist in vacuums with little guidance on how to use them as a suite that work with each other. As stated previously, these tools contain many different Strategies which could leave designer's perplexed as to which Strategies are best suited for their goals and business, which ones they should implement, how to implement and in which order. Organizing Strategies by innovation level and impact could potentially assist designers in choosing which product level innovation strategies best suit the long-term goals and vision of a designer or business. The last barrier is tool appeal. Tools must be appealing to designers to use. This includes aspects such as format (app, cards, model, text), aesthetics (use of graphics and symbols over text) and ease of use. The literature cites the most popular and appealing tools to use were those in a card format (Wölfel \& Merritt, 2013)).

Learning is required to use the tools and implement Strategies recommended by the tools. Research and reading are necessary for developing fundamental knowledge and tool usage is dependent on a designer's level of knowledge. Learning and using tools is time-consuming. This includes planning, implementation, action, monitoring and sustaining a sustainability plan. For designers, this adds time and cost to activities such as sourcing materials, vetting factories and auditing/monitoring factories and their supply chain. The financial commitment and resources are quite high as they require costly initial investments upfront before any product is even sold. This can be a high-risk endeavor for MSE businesses. The complexity of sustainable fashion itself can be an overwhelming task for designers, especially sustainable fashion entrepreneurs. Given this complexity, we find the guidance or "how-to" component of the tools to be minimal. All of the tools contain many Strategies and a variety of forms of ancillary information. This typically entails providing descriptions, definitions, examples and further readings but lacks the "how-to" component to integrate these strategies into a fashion business and design practice.

Tools that were seen as easiest to use as an independent or MSE designer were those that provided examples, case studies and provided further reading. These tools are in a card format and available as free downloads. The format of the Assessment tools is suitable for experts in sustainable production and supply chain management rather than designers. The Universal and 
Participatory tools are better suited for MSE designers while it was found the Assessment tools are more suitable for large corporations. Large corporations were involved in the development of some of the tools (e.g., Higg Index, C2C). Only large corporations have the capabilities and resources to implement dedicated teams for their use.

\subsection{Conclusion}

Given the variety of tools available, several recommendations are offered for tool developers based on the fitness for purpose of the tools. First, there are advantages for tools to be developed to work with other tools. Lofthouse (2006) highlights the significance of tool suites as "nothing does everything" and no tool "offers a one-size-fits-all solution" (Knight \& Jenkins, 2009, pg. 557). Second, tools should be targeted to how designers work; they must speak their language. Tools that tended to be more visually based and intuitive were those that were developed in conjunction with designers (e.g., Sustainable Design Cards, Ted's Ten). Third, context is extremely important, and tools should identify the best context for their use or be developed for specific contexts (e.g. workshops, daily use, strategic planning). Designers need to consider and embed transformational Strategies if the industry is to enact the change needed for a sustainable fashion system. Fourth, Strategies categorized at the Transformative level are minimal in number and developers should consider including higher level Strategies to assist designers with the transition to a sustainable fashion industry. The final recommendation would be to rethink the role of the designer beyond a creator of garments; MSE designers often fulfill multiple roles (Kozlowski et al., 2018) and tools should acknowledge these. This can include tool support, training workshops or opportunities to work with academic researchers in tool development.

This study contributes the first comparative analysis of the fitness for purpose of the existing tools for sustainable fashion design, resulting in the identification of three tool archetypes: 1 . Universal, 2. Participatory, and 3. Assessment. To analyze the tools, we developed an innovation framework to categorize the Strategies contained within the Tools. Four levels of innovation have been proposed: 1 . product-level, 2. product service social systems level, 3. systems level and 4. resilient, adaptable and circular level. We found that the majority of the Strategies presented by the Tools were limited to product-level innovations. The impact of Strategies categorized at the product-level is limited to improvements rather than transformational change. Assessment tools predominately prescribed to Strategies that are categorized as product-level innovation and addressing the environmental dimension of sustainability. The interrelation between the archetypes, the innovation framework and strategies reinforce the notion that 
sustainability can be approached at multiple levels. For designers, sustainability must be approached in manageable pieces (product-level innovation and associated strategies) but are related and moving towards the bigger systems thinking vision that a designer has established (through to RAC innovation and associated strategies). By establishing a vision and long-term goal that seeks transformational impact, a designer can select appropriate and manageable strategies, directly related to their long-term goal, that improve and challenge the current system. Designers are therefore working within this duality of a very specific focus embedded within a systems thinking big picture lens-moving within the two.

The Universal and Participatory tools contained Strategies found within all innovation levels and are most suitable for MSE designers. Therefore, in terms of fitness of purpose, the Universal and Participatory tools are deemed to be most appropriate for designers based on the results. The Universal and Participatory tools are also well-suited for use in conjunction with Assessment tools. Through the analysis, we have identified barriers, opportunities and further recommendations for tool developers. We have also proposed a new sustainability framework specific for fashion that includes five dimensions: 1. Environmental, 2. Economic, 3. Social, 4. Aesthetic and 5. Cultural. We find that to build a sustainable fashion industry, designers should consider and incorporate the proposed five dimensions of sustainability for fashion and the four levels of innovation throughout their design process. 


\title{
4. A CASE STUDY OF SUSTAINABLE FASHION MSE'S AND THEIR SUSTAINABLE DESIGN PRACTICES
}

\begin{abstract}
This study investigates how sustainable fashion designer entrepreneurs who own and operate a micro-to-small enterprise (MSE) integrate and foster sustainable practices into their design process. This study looked at designer's knowledge and use of sustainability practices, business practices, design process and tools. Thirty-seven design entrepreneurs and sustainable fashion experts were interviewed. Themes and patterns into the types of sustainability strategies used and challenges were identified through a qualitative content and thematic analysis. Identified strategies used by design entrepreneurs include reducing waste, zero waste pattern drafting, material selection, ethical production and alternative business models. Many of the participants operated outside of the traditional fashion system which provides the design entrepreneurs with the space for creativity, flexibility, collaborations, innovation and control over their brand and business. This, however, also creates challenges with respect to sourcing, stigma, a niche categorization, financing, greater costs (lower margins), customer acquisition, market access, marketing exposure, and competition with pricing (fast fashion). Overall, MSEs have been proactive in integrating sustainability into their businesses, however they need further support with the resources, systems and structures to succeed in their endeavors for a sustainable fashion system.
\end{abstract}

\subsection{Introduction}

The contemporary fashion system is contradictory to the needs and development of sustainability. Fashion is now a system characterized by speed, global production networks, perceived scarcity, branding, and gross over-production and over-consumption of apparel, footwear and accessories. It is a US\$2.5 trillion industry that is responsible for producing upwards of 150 billion new garments a year (Kirchain, Olivetti, Miller, \& Greene, 2015). The intense worldwide growth and popularity of fashion is a relatively recent phenomenon, occurring over the last 20 years or so. The early 21 st century has ushered in a period of dramatic globalization, falling costs, streamlining of operations, and growing consumer spending, as well 
as the rise of fast fashion, social media, a celebrity fetishization and street style. The industry is now dominated by international firms that favor the fast fashion model.

"Fast fashion" is a catchall term that prefers using efficient, sub-contracted, flexible supply chains to produce trendy clothing at the lowest price (Reinach, 2005). In 2014 the average consumer bought $60 \%$ more clothing than in 2000 but kept each garment only half as long (Remy, Speelman, \& Swartz, 2016). This sharp increase has had detrimental effects as clothing production is extremely resource intensive - consuming enormous amounts of water, energy and toxic chemicals (Caniato, Caridi, Crippa, \& Moretto, 2012; Remy et al., 2016). Clothing use and disposal are becoming increasingly problematic as the laundering of synthetic textiles is identified as the largest contributor to primary microplastic contamination of the world's ocean (Boucher \& Friot, 2017). Nearly three-fifths of clothing produced globally ends up in a landfill or is incinerated within one year of being made (Remy et al., 2016).

In addition, there are many social impacts associated with the fashion industry. For example, fashion often has long and complex supply chains that are rife with unsafe conditions, and the use of child labour and other exploitative practices, especially for women and girls (Minney, 2017). The fast fashion model is characterized by these supply-chains that epitomize "flexibility" by foregoing ownership of manufacturing facilities in favor of sub-contracting. This model, however, creates conditions for exploitation as these firms have essentially removed responsibility and accountability alongside with ownership of their supply chains. As a response to the damaging effects of dominant industry practices, a slow fashion paradigm has emerged led by sustainable fashion micro-and-small sized enterprises (MSEs).

The purpose of this study is to explore MSEs in the sustainable fashion system and enhance the knowledge on current and potential practices as it relates to developing a circular closed-loop sustainable fashion system. This research investigates the creative design practices of sustainable fashion MSEs. It aims to understand how they define sustainability and how their concept of sustainable fashion is integrated into their design and business practices, types of tools and resources used and what type of challenges they encounter. The following research questions are investigated: 1) Which, if any, sustainable fashion design strategies are MSEs implementing into their design practices? 2) What are the barriers that sustainable fashion MSEs encounter?

Understanding of sustainable design varies depending on perspective and context as new aspects of its complexities are revealed with every new angle of view (Walker, 2006). In 
particular, we demonstrate that sustainable fashion MSEs have an innovative approach to sustainability characterized through their use of an anti-fashion calendar and direct-to-consumer (D2C) business model, a commitment to fair and ethical garment production, collaboration, a zealous commitment to reduce waste and a revalorization of craftsmanship and working with artisans. These designers are driven by personal beliefs and values that are manifested through their entrepreneurial activities and practices as they seek to compete with the paradigm of fast fashion. Slow fashion is the preferred paradigm of these designers as they seek to challenge the dominant industry norms and practices and create consumer awareness regarding responsible production and consumption of fashion products. This study contributes new insights and understanding into the design and business practices of sustainable fashion MSE. Themes and patterns into the types of sustainability strategies used and challenges were identified through a qualitative content and thematic analysis.

\subsection{Background}

There is no mutually agreed upon definition and/or concept of sustainable fashion as many interpretations exist (Armstrong \& LeHew, 2011; Fletcher, 2008; Fletcher et al., 2012; Henninger, Alevizou, \& Oates, 2016; Jestratijevic \& Rudd, 2018; Jung \& Jin, 2014). Since the inception of the term, there has been an evolution to the concept and how it is defined in the academic literature (for example, Armstrong \& LeHew, 2011; Henninger et al., 2016). For the purposes of this paper, sustainable fashion will be defined as: the profitable design, production, distribution, and end-of-life reuse, recycling, or disposal of fashion that promotes circular systems, ecological integrity, human flourishing, connectivity, and shared values while minimizing negative and maximizing positive impacts on both society and the natural environment. The approach to developing and fostering sustainable fashion by fashion companies is dependent on how they perceive, conceptualize and define sustainable fashion.

Fashion companies and retailers have made a number of initiatives and commitments towards addressing the negative impacts of the industry (Kozlowski, Bardecki, \& Searcy, 2019). These primarily take form of: 1) individual companies pledging initiatives to address negative environmental or social impacts such as reducing their carbon emissions, water footprint or virgin material use, providing a living wage, or banning the use of animal products and toxic chemicals; and 2) companies aligning commitments with institutional projects such as Greenpeace's Detox My Fashion (Greenpeace, 2019), Carbon Disclosure Project (Carbon Disclosure Project, 2019), Fashion Revolution (Fashion Revolution, 2019), or Global Reporting 
Initiative (Global Reporting Initiative, 2019). The narrative of sustainable fashion is largely centred around leading international firms' initiatives and the institutional projects with which they are aligned.

A number of tools for sustainable fashion design have been developed to aid designers in the integration of sustainability into their design practices (Kozlowski, Searcy, \& Bardecki, 2019). The premise behind these is that one of the first steps to tackling sustainability within one's supply chain is to determine a baseline assessment of your products' impacts. This allows one to quantify a starting point, set meaningful targets and goals and put effective programs in place to achieve those targets and goals. It is then equally important to demonstrate, in a transparent way, that targets are being achieved and progress is being made.

Tools have different aims and scopes to help designers in a variety of ways from impact assessment to facilitating a co-design process (Kozlowski, Searcy, \& Bardecki, 2019). This includes using combinations of tools at different points of the design process and throughout one's supply chain in collaboration with other stakeholders (Kozlowski, Searcy, \& Bardecki, 2019). Using design tools can be a critical starting point for a sustainable fashion business as you can not improve what you can not measure.

The academic literature and popular press regarding the differing types of sustainability orientated tools, strategies, and innovations available to designers are continuously expanding and evolving. There is a trend to implement various forms of sustainability by both small and large fashion companies (De Brito, Carbone, \& Blanquart, 2008). Still, little is known about how sustainable fashion firms actually put the principles and strategies of sustainability in practice. Literature has documented the impressions of fashion designers (Palomo-Lovinski \& Hahn, 2014) and sustainable fashion experts (Pedersen \& Andersen, 2015) and documented case studies of small- and medium-sized sustainable fashion companies' practices (Lawless \& Medvedev, 2016) and competitiveness (Battaglia, Testa, Bianchi, Iraldo, \& Frey, 2014).

There is no consensus among designers about whom sustainable practice should be aimed at and where it would have the greatest impact (Palomo-Lovinski \& Hahn, 2014). Designers do not currently feel enabled to create change when it comes to sustainability (Palomo-Lovinski \& Hahn, 2014). Pedersen and Andersen's (2015) survey of global experts found current approaches to sustainability are limited and fail to address fundamental challenges linked to the traditional linear business models and overconsumption. These observations confirm that efforts towards 
sustainability within fashion are still piecemeal and reactive (Kozlowski et al., 2018). Despite the recent emergence of sustainability-orientated innovations and strategies in fashion, little is known about the relevance and application of such strategies for micro- to small-sized enterprises (MSEs). MSEs in the fashion industry have recently been identified as drivers for sustainability as they have more opportunity to connect to the whole product lifecycle (DeLong, Goncu-Berk, Bye, \& Wu, 2016).

A paradigm of slow fashion has begun to emerge as an alternative option to the frenetic pace that dominates the industry today (Bhardwaj \& Fairhurst, 2010; Ozdamar Ertekin \& Atik, 2015; Pookulangara \& Shephard, 2013). Slow fashion is a fairly recent development in both practice and theory. Contemporary researchers vary in their definitions (Bhardwaj \& Fairhurst, 2010; Cataldi, Dickson, \& Grover, 2010; Clark, 2008; Fletcher, 2008, 2010; Jung \& Jin, 2014; Pookulangara \& Shephard, 2013) and therefore have yet to be widely accepted as they continue to evolve as slow fashion becomes more prevalent. One such characterization of slow fashion is a philosophy of balance and mindfulness "in which designers, buyers, retailers and consumers are more aware of the impacts of products on workers, communities and ecosystems" (Fletcher, 2008, pg. 173). There is emphasis on giving rise to different relationships between designer and maker; maker and garment; garment and user through a heightened awareness of quality, stakeholders and speeds. Speed is not referencing that slow fashion stands in opposition to fast fashion, as there is no dualism that exists. Slow fashion is a discontinuity from the goals and values of fast (growth based) fashion (Fletcher, 2008). As Fletcher (2008) notes, "recognizing and designing with speeds other than just a fast commercial pace takes the pressure of time" (pg. 173). The concept of designing slow fashion is characterized by high quality, workmanship, values, balancing speeds, regional production networks, transparency and fair labour conditions (Fletcher, 2008; Pookulangara \& Shephard, 2013). A key manifestation of this sector is the emergence of design entrepreneurs operating sustainable fashion MSE.

If design is to become central to sustainable development within fashion, it is essential to understand how designers currently work. Therefore, this study seeks to develop an understanding of MSE fashion design practices and fashion design entrepreneurs' knowledge and use of sustainable design practices. It is important to note that MSEs cannot be treated as smaller versions of their larger counterparts (United Nations, 2019) and therefore require approaches, initiatives, tools and resources tailored to the nuances of running a sustainable fashion MSE. 


\subsection{Method}

To understand the sustainable fashion design strategies implemented by MSEs in their design practices, a qualitative analysis approach was chosen. To select participants, purposive sampling was used: the deliberate choice of participants due to the qualities they possess. This is effective when investigating the knowledge of experts in the field (Lavrakas, 2011; Tongco, 2007). The key criteria were that designers identify as a sustainable fashion designer/brand and they operate at the scale of an MSE (i.e., less than 50 employees).

Instagram was used as a solicitation tool for participants. Instagram is one of the most influential social media platform utilized by the fashion industry (Rocamora, 2017). Smaller brands have a seemingly stronger presence on Instagram than on websites, likely due to Instagram being a free platform that is easy to use. Searches based on the hashtags \#sustainable fashion, \#sustainable fashion design, and \#sustainable fashion brand were used to compile a list of potential sustainable fashion designers.

Participants were contacted through Instagram or the contact information provided on their platform or websites. Thirty-seven participants were recruited. Of these, 30 self-identified as design entrepreneurs (DEs) who own and operate MSE focused on sustainable fashion. Design entrepreneurs are fashion designers who own and operate a small business where they are responsible for the majority of duties, including those related to running the business, sourcing materials, merchandising, working with manufacturers, distributors and retailers and traditional design responsibilities. Seven others were experts in field. The experts were consultants, buyers for sustainable fashion boutiques, professors who are also trained fashion designers, and/or those working for not-for-profit sustainable fashion organizations. The experts were able to provide additional context and knowledge regarding sustainable fashion practices. Participants were distributed geographically as follows: 21, United States; 9, Canada; 6, Europe; and 1, Australia.

The design entrepreneurs selected for this study owned and operated independent MSEs. With the exception of one male design entrepreneur who did contemporary menswear and one MSE that offered both womenswear and menswear, all of the MSE's were contemporary womenswear brands owned by women design entrepreneurs. They all operated out of studio spaces - a few with in-house small-scale production facilities. Their business operations consisted of two retail streams: 1) direct to consumer online, and 2) wholesale accounts with high end boutiques, department stores and other online stores. Studio space is preferred as their 
creative hubs where they can research, conceptualize, develop and execute designs and conduct their business. The MSEs exhibited many attributes: flexibility, independence, informality, innovative behaviours, highly motivated employees, and highly personalized management styles. There is a sense of reconciling personal fulfillment with professional achievement among the sustainable fashion design entrepreneurs.

Interviews were used to address the research questions - a familiar strategy for collecting qualitative data (DiCicco-Bloom \& Crabtree, 2006). The interviews were conducted individually and adopted a semi-structured format. Interviews took place from 2016 to 2018 either through Skype or in person and lasted between 1-2 hours. All interviews were audio recorded and transcribed verbatim. To identify themes, transcripts were coded and sorted based on data relationships (Cope, 2005). Once the data were categorized, relationships and themes were identified. Thematic analysis is a broadly used qualitative method for identifying, analyzing and reporting patterns (Braun \& Clarke, 2006, 2019). Themes are identified across an entire data set rather than within a single data item. Braun and Clarke (2006) define a theme as "captur[ing] something important about the data in relation to the research question, and represents some level of patterned response or meaning within the data set" (pg.10). Many iterations of coding took place to categorize and identify relevant themes.

In addition to interviews, the data set for the thematic analysing includes information gathered from the design entrepreneurs' online presence. This included their social media platforms such as Instagram, websites and popular press articles written about the designer's and their businesses. This provided additional information regarding the branding and business of the design entrepreneurs. Websites contain information about the company such as the "about" page, sustainability practices and overall branding. Instagram provides insight into the day to day branding and marketing activities, consumer engagement and awareness put forth by the design entrepreneurs through their posts. All of the information collected provided a more holistic perspective about the MSEs. All interviewees are treated as anonymous.

\subsection{Results and Discussion}

This section presents the results of the interviews and discusses the themes and implications of the responses. The discussions with the designers have been organized under the following headings: 1) designer's definition and conceptualization of sustainable fashion, 2) business practices, 3) design practices, 4) information, 5) branding, 6) incubators and accelerators, and 7) 
manufacturing and sourcing. Each section summarizes designer's responses, identifies themes among the responses, the implications of the findings and any challenges the designer's encounter.

\subsubsection{Sustainable Fashion Definition and Conceptualization}

Although much has been written under its rubric, sustainable fashion itself remains a diffuse and nebulous term. We asked the designer entrepreneurs how they define sustainable fashion for themselves and their brand. From the discussions, we were able to get a sense of how they conceptualized sustainable fashion through their design and business practices. They did not respond with formal definitions of sustainable fashion but rather expressed their guiding values and/or an issue they had identified as important for them. Each designer had a distinctive crafted version of what sustainable fashion meant to them. Their definitions were grounded in values and beliefs that informed how they practice sustainable fashion design and operate their business. The specific concerns they had regarding how fashion operates (e.g. water pollution, overconsumption or unethical labour practices), shaped the decisions they have made in defining what kind of sustainable fashion brand they are. Table 4.1 contains a selection of sustainable fashion definitions from the design entrepreneurs.

\section{Table 4.1: Sustainable fashion definitions}

\begin{tabular}{|c|c|}
\hline Designers & Definition \\
\hline DE1 & $\begin{array}{l}\text { It has to be sustainable for the environment but also for the people involved (human rights). As } \\
\text { small and as local as you can keep your supply chain seems sustainable to me }\end{array}$ \\
\hline DE2 & $\begin{array}{l}\text { People talk about the holistic approach. And, like I said earlier, this whole language thing is so } \\
\text { confusing. Am I a sustainable designer and does that mean that I do not involve myself in other } \\
\text { areas or does sustainability cover everything? And in all honesty I don't know. I really feel that } \\
\text { sustainability is for me - this idea of being able to not exploit resources to make something that is } \\
\text { beneficial for the present and for the future. To actually create in a beneficial way, I think for me is } \\
\text { sustainable. So beneficial [is] being for people and not just me or the person wearing something; } \\
\text { beneficial for the person growing or developing the fabric or the material or the raw fibers; } \\
\text { beneficial for the person producing, beneficial for the environment. }\end{array}$ \\
\hline DE4 & $\begin{array}{l}\text { I guess the best version of how you can do it. For me it is manufacturing as locally as possible and } \\
\text { using high quality fabrics and styles that are going to have longevity.... So that's how I think of } \\
\text { sustainability for my business. }\end{array}$ \\
\hline DE7 & $\begin{array}{l}\text { To me something that is sustainable is something that will make whatever it is equal or better in } \\
100 \text { years than it is today. That to me is the definition. I think that is kind of the filter we run } \\
\text { everything through before we decide on things-is this going to make the people we work with, the } \\
\text { places we work at - that kind of stuff-better or worse in } 100 \text { years. And if it's better, [that is] the } \\
\text { right trend }\end{array}$ \\
\hline DE12 & $\begin{array}{l}\text { First and foremost, [we are] plant-based. [We use] only plant-based fibers. And also producing as } \\
\text { close to where I'm based as possible, [and] omitting the distance things have to travel... get to } \\
\text { where I'm making it. }\end{array}$ \\
\hline DE14 & $\begin{array}{l}\text { Capitalization has brought us [a] huge proliferation of things: commodities and unnecessary things. } \\
\text { What I'm exploring is how do we just keep the essentials, so that our lives would be simpler, and we }\end{array}$ \\
\hline
\end{tabular}




\begin{tabular}{ll}
\hline Designers & Definition \\
& $\begin{array}{l}\text { are not using as much resources as we are now? Leaving a smaller footprint on the Earth and using } \\
\text { local resources as much as we can-to me that's what it is. }\end{array}$ \\
\hline DE19 & $\begin{array}{l}\text { It is really just about working in a forward-thinking way, I think, with just consideration [of] the } \\
\text { context and the ramifications of the decisions we all make, especially as designers and manufacturers, } \\
\text { because our decisions have further ranging impact. I guess in the case of my sort of company's } \\
\text { philosophy, the idea that you can create something that appeals to somebody enough that they will } \\
\text { wear it over and over again, and style it differently, and just kind of make it an essential part of their } \\
\\
\text { wardrobe and not feel this need to buy a new dress next Friday night because they're so obsessed with } \\
\text { this one that they're going to wear it again with a different jacket or something, and just change things } \\
\text { up a little. }\end{array}$ \\
\hline The main thing that I focus on is trying not to produce anything that is not necessary. We don't pre- \\
produce garments and then hope that they will be sold at some point but that garments are only \\
produced when someone wants to actually buy one. So that is the main part and then the secondary \\
part for me is working only with mono-materials. No sewing thread, no zippers no additions to the \\
fabrics.
\end{tabular}

Themes that consistently arose as issues in the designers' responses were: waste reduction, local production, longevity, aesthetic sustainability, sustainable materials, living wage, balance and transparency. Reducing waste was one of the most prevalent themes, a significant majority of participants felt very strongly about the various ways they could reduce waste, either through materials and/or methods/processes, especially if they can avoid producing anything they see as not necessary. Increasing the lifespan of garments was seen as correlated to reducing waste for many of the designers. Keeping their supply chains as short and as local possible was another prevalent theme. With the exception of a few designers who produce some of their merchandise offshore, as a whole, the design entrepreneurs favoured local production. Reasons for supporting local production included being able to ensure ethical practices, transparency and living wages. It was also considerably easier to develop a relationship with the stakeholders in one's supply chain. These sorts of concerns were present among even those with non-local production. For example, one design entrepreneur (DE13) has her sweaters manufactured by local artisans where she sources the wool. This allows her to keep her supply chain short and manageable as all the production is localized to one area.

The conceptualization of sustainable fashion was shaped by the design entrepreneurs' individual core beliefs and values. For example, designers who had concerns over animal rights had well-defined parameters as to what types of materials or processes were acceptable for them (did not harm or use animals or their by-products). This was also evident in how they conceptualized sustainable fashion, as their responses included phrases such as "plant-based materials" or "no animal products". For others, their conceptualization of sustainable fashion 
involved ideas around durability and longevity, and in moving away from the traditional fashion calendar, timelessness, seasonlessness and trans-seasonality.

Many of the designers disliked the term "sustainable fashion" for a variety of reasons. A significant majority of the design entrepreneurs felt the term is used very casually, causing it to become meaningless: a buzzword. They also felt the term traps them into a niche categorization that still suffers from stigmas. As DE19 explained, "I'm worried about that term. It has been used so much that it has lost a lot of its meaning and at the same time there is this sort of stigma around the whole idea of sustainable fashion, where, for a lot of people, as soon as it is marked sustainable they don't really consider it fashionable." This is seen to cause difficulties with branding, communicating and informing their consumers. As Expert 4 noted:

[Sustainable fashion] is a myriad of statements.... It is sort of almost meaningless in a way for you to use a term like sustainable designer. You have to decide as a human being, as a person, what is important to you or what you will support and what you are able to support... as a brand. As a designer you have to be specific about what it is you are doing.... You call it "zero waste"; that is what you can call it because that is what you are doing.

It is clear from the variety of responses that it was seen as valuable to conceptualize sustainable fashion by what is being achieved, such as being able to label your brand as being zero waste, vegan, ethical, carbon neutral, fair trade, or organic. One designer (DE18) stated, 'I've sort of moved away from sustainable fashion. I am focusing more on responsible fashion.... I do not think everybody identifies with the concept of sustainability.... [It is] kind of elitist.... Everybody gets [responsibility], everybody wants to be responsible for the most part." Overall, it is seen that moving away from the term sustainable fashion would help combat confusion over the term and can address the branding and marketing issues designers face. Sustainable fashion was seen as a culture: this was evident through how they conceptualized sustainable fashion for themselves and their brand, how this translated into their business and design practices and was truly a value and belief that drove their lifestyle. They are all working to redesign the system.

\subsubsection{Business Practices}

Discussion took place around the business practices of the design entrepreneurs, how the principles of sustainability are embedded, and the strategies they use. Four types of issues related 
to their business models arose: 1) anti-fashion calendar; 2) wholesale and direct-to-consumer retail strategies; 3) sustainability as an added-value; and 4) collaboration. Expert 4 perfectly sums up the trend that is occurring in how MSEs are choosing to do business, "small companies: ... ultimately, they are more creative, and more and more of them do not feel the need to follow the rules of the system that is in place".

The traditional fashion calendars works with "seasons" where retailers expect multiple large deliveries throughout the year and far in advance of the actual season the clothes are intended for. For example, the first spring delivery arrives in-stores in January and Fall deliveries begin to appear in end of June/July. The industry's response to the fast fashion model was to create new seasons such as Resort, pre-Spring and pre-Fall. Overall, the traditional industry calendar is more demanding for fashion businesses with the intensification of speed and multiple seasons/collections presented throughout the year. For MSEs this traditional model is not sustainable economically or psychologically and leaves little time to be creative and do research.

Many of the designer entrepreneurs do not want to produce so many collections at a frenetic pace only to have them marked down. For example, one designer (DE12) explained that when summer clothing is retailed in January/February when nobody buys them, they end up on discount before the summer arrives. This has further implications, "Stores are marking stuff down, which puts the pressure on me to lower my prices". This is problematic when a designer sells both online and manages wholesale accounts where they must maintain consistent pricing through multiple distribution/selling channels.

The business model that the MSEs chose was directly related to how the designers defined and conceptualized sustainable fashion. Many of the designers preferred working with what they described as an "anti-fashion calendar" model. The traditional fashion calendar requires working far in advance to deliver seasonal collections far in advance of the actual season. Characteristics that define an anti-fashion calendar model as described by the designers include: seasonless garments, drops or capsule collections, exclusive collaborations, working in-season and smaller production runs. By working with more seasonless clothing and adopting the "drop" or capsule collection concept, designers can work closer to the actual season of delivery or no season at all.

This works best with local production. Designers noted that they are better able to respond to consumer demand and predict more accurately styles or colours that will have a full-price sellthrough. Many of designers only produced on demand or made to order in order to further reduce 
waste by not producing anything that was not already sold. This also helps reduce storing of stock and/or retailers who return unsold merchandise to brands after the season.

The move towards an anti-fashion calendar is very much both a business and design strategy. The anti-fashion calendar strategy is easier to realize using direct-to-consumer models than wholesale. Designers recognize that when they sell wholesale, they do not get to dictate the selling calendar. Almost all the designers had a business model that included a mix of direct-toconsumer (mainly online) and wholesale business models. The direct-to-consumer model gave them flexibility, insight into the consumer and consumer preferences and let them better control inventory to reduce on unnecessary production or unsold merchandise. Wholesale sales provide visibility to the brand when retailed in specialized boutiques, high-end retailers and/or department stores.

With the wholesale model, the designers felt communication with buyers was extremely important. Designers wanted to ensure the buyers understood the benefits of their sustainability practices as value-added for their products. The following five major reasons were cited: 1) pricing, 2) awareness and information regarding nuances of sustainable design, 3) competitiveness, 4) garment care, and 5) reorders. Because sustainable fashion clothing tends to be more expensive, explaining to customer why the garment sits at a particular price point aids with awareness and information regarding sustainability. The designers were quick to note that the style and aesthetic is what sells, not the sustainability. However, when you are a relatively unknown MSE and do not have the power of a recognized brand, this value-added sustainability component is important to communicate. The value-added of sustainability is a key competitive factor for MSEs (Battaglia et al., 2014).

In sustainable fashion, many of the methods and practices used do not always result in a consistent appearance. The introduction of mass-produced products sought to eliminate the nuances that are celebrated with hand-crafted products, as a consistent replicable appearance is the goal and what consumers have become accustomed to. As explained by many of the designers, high-quality is favoured and products are very well made. Unlike traditional product lines which tend to be very consistent in appearance and synonymous with quality control, sustainable products may not be consistent in appearance, shape, colour or seaming. Sustainable fashion designers tend to work more with craftspeople and artisans or using artisanal techniques such as natural dyeing; this is known to result in variation in tones and hues due to a variety of factors. Designers noted that informing the buyers and consumers about these nuances is 
significant. Equally important is communicating the care of the garment so that the garments last longer. Buyers and retail sales staff act an important touch point in the selling of sustainable fashion garments, ensuring consumers are informed about how to care for those garments for longevity.

When dealing with wholesale accounts, reorders are common; for designers who use deadstock materials or zero waste techniques this can be problematic. All of the zero waste designers stated the importance of ensuring buyers are made aware beforehand that certain styles may not be available for reorder. When using deadstock materials, the same material may not be available for a reorder. Or when using deadstock or zero waste, no two pieces may look alike.

Added value through service was another business practice of the sustainable fashion MSEs. Many felt that for sustainable fashion to move forward, collaboration and sharing through opensource platforms is key. This idea of fostering community, employing community and offering services to promote consumer engagement through making was inspiring. The design entrepreneurs were creative in how they approached selling in a different way. This appears most important in two situations as highlight. For example, in order to combat the higher costs associated with sustainable fashion, some of the designers would sell patterns that they had developed for a very affordable price, providing consumers with a choice: to buy the ready-made product, or to make it themselves with the pattern. Two of the designers strongly encouraged their community to "hack" these patterns and share them to encourage making (maker movement). They found, echoing emotionally durable design, when consumers are engaged in the making, they value the product differently, increasing its longevity in their wardrobe. One designer even sold previously used wool from knitted garments that she would unravel herself; citing this is a practice most people do not have time for, but she saw it as a way to reduce and reuse while encouraging making. Future plans include employing the local community to unravel for her.

Since most of the designers were attempting to move away from how the industry traditionally worked, they found new alternatives, many based on collaborations with each other. There was an advantage to creating a "collaborative identity" in the sustainable fashion space. This was especially true for those design entrepreneurs who belonged to an incubator or accelerator. Advantages to joining included cost savings, customer acquisition, marketing exposure, and challenging the stigma of while communicating the value of sustainable fashion. Designers host their own events such as pop-up retail shops or putting on their own market week 
rather than participate in the large trade shows. MSEs face many obstacles with financing; collaborating is one of the easiest ways to cut costs such as sharing of studio space, ordering supplies in a bulk order, or dealing with the costs associated with hosting a pop-up shop. They are also opting out of participating in fashion shows - another costly traditional notion of selling fashion. Instead, design entrepreneurs are opting for putting on collaborative events that challenge how the industry traditionally markets and distributes fashion. Hosting events with other MSEs enhances marketing exposure, resulting in greater potential for customer acquisition while sharing costs. This also allows for the designers the opportunity to converse with potential customers or buyers and explain the benefits of their sustainability practices and designs. DE13 described their experience:

I participate in more collaborative tradeshows. Instead of going to a main tradeshow, I grouped together with five other brands and we rented space: mainly because I was broke and the tradeshows are expensive but, then again, by doing that I ended up by accident showing with brands that were really focusing on sustainability.... Through meeting them and learning more about their process, I could [begin to] source yarn that is organic; it just kind of opened my eyes a bit more to it.... This past season we had 15 brands and we rented the space for seven days and it was really nice. Any buyer that came by... were specifically coming to meet with somebody and probably place an order. It would be a waste of time to go there if they were not pretty interested; ... and all the brands had a similar aesthetics, so they were much more willing to walk around. It was really chill; the space was really nice, and we had really good snacks and wine. It was just a lot better than normal tradeshows. It was a really good season this year. I think it started to gain more and more interest.

The collaborative nature and identity of these design entrepreneurs challenge industry norms and beginning to dismantle the stigma of sustainable fashion and lifestyles while creating greater awareness.

\subsubsection{Design Practices}

Throughout the discussions with the design entrepreneurs about their design practices, it was evident that the choices the designers made in using certain sustainability strategies reflected their unique conceptualizations of sustainable fashion. The most common strategies used by the design entrepreneurs are summarized in Table 4.2. 
Table 4.2: Most commonly used sustainable strategies by the design entrepreneurs

\begin{tabular}{l}
\hline Sustainable fashion strategies \\
\hline Zero waste design/reducing waste \\
\hline Material selection: particularly preferred were natural fibres as they can biodegrade \\
\hline The use of core styles and silhouettes \\
\hline Ethical production \\
\hline Transparency \\
\hline Working with artisans \\
\hline Incorporating craft techniques \\
\hline High quality and durability \\
\hline Working around concepts of minimalism, versatility and seasonless \\
\hline Co-design \\
\hline Emotionally-durable design \\
\hline
\end{tabular}

One of the most common strategies was reducing waste. As DE18 lamented, "I see the amount of waste that happens when you are cutting out dresses and the amount of fabric that you cannot use. It really bothers me. I do not know what to do about it, there is not really a lot you can do. So, you are constantly kind of making trade-offs, asking what the best way is that I can do it." Designers sought to reduce waste through: material selection (e.g. deadstock), using core styles and minimalism (promotes longevity), producing on demand, trying not to oversample or overbuy materials, following an anti-fashion calendar, and incorporating repair-ability and aesthetic sustainability.

Over one third of the designers incorporated some sort of zero waste strategy (e.g., by using zero waste pattern drafting and repurposing of scraps). Two of the designers approach zero waste design through working with square pieces of fabric that they manipulate using pleats and darts to achieve desired shapes. There are a variety of choices these designers make to integrate sustainability and the creativity required which differ from that of a traditional designer:

I usually take pieces of fabrics and I start to put them on the dummy, and this is how it works, and then I kind of create some shapes, and then after I have a small production.... When I have these raw prototypes, we go through them together and we talk about finishing and fitting and how to make an actual product out of it. It is very creative.... I like to use [straight] angle... I am free from this traditional pattern making. I just let my imagination go.... I like the way that I can create some really different looks, something 
totally fresh with these rectangles.... I think my zero waste... is really something that works well in a big production... because then, when you have rolls of fabric, you can really easily calculate everything with a computer and just cut it in a way that there is no waste. (DE20)

Another designer weaves fully fashioned garment pieces eliminating any fabric waste; an innovative approach to zero waste design:

It always starts with the weave: the planning of the weave. I do not really have a design in my mind. I know the shape that I want to make... so this is all shape-woven again.... All the pieces of clothing are from the loom. There are no incision marks whatsoever when I construct the garments. I do have to cut off the excess, but... this is where it starts, from a drawing, and then I calculate. I dress... and I weave; and as I weave, I have to make some tweaks because sometimes [the pattern] does not work. (DE14)

Material choice is a central factor in developing sustainable products as was evident from the designers' responses. All of the designers discussed their material choices and the implications of those choices. The most common material choice was to use natural fibres. Designers were adamant about not wanting to support the overabundance of plastics that exists. They felt that if they are not able to recycle textiles or even recapturing them through a take-back program; at least natural fibres will eventually biodegrade when garments are discarded. The use of deadstock or end-of-the-roll fabrics was quite common among many of the design entrepreneurs. The designers acknowledged the use of deadstock fabrics as a waste reduction strategy when sourcing materials.

Working with artisans and/or artisanal and craft techniques was another notable sustainable design strategy. DE15 stated "I try that every collection that I do has something artisanal; made in an artisanal way". Examples include using natural dyeing, working with button-makers or knitters in Peru. The designers saw a natural association with sustainability and many benefits in supporting the arts and crafts community. Preserving and valuing the artisan sector is an issue of global sustainability and cultural knowledge. Two of the designers were deeply engaged with their supply chain promoting working within a fibreshed. Designing and planning with materials that are only found within a local fibreshed has many considerations as detailed here by one designer: 
I do not have the luxury of designing and going, "I like this [material]... Let's go find it." I have to gather my materials first and then group them [e.g. wool, alpaca or cotton] and then see. "Okay... this [material] cannot do this. This [material] can do this. Okay we can do that." And then I develop [designs] around what [materials] exist. The neat part is that the collections are only going to get better as the supply chain gets better. So, you are going to actually see some real progress as the materials evolve and improve. So, as our fabrics get drapier and finer, the dream is to do wool suiting one day.... When a lot of people ask me about the timelines and the cycles of things, I say, well, alpaca is finished being shorn in June, it must be mixed with wool because alpaca is too slippery; it falls apart it has to have wool, or nylon. We try not to use nylon. So, we are going to wait for wool. Well wool is not finished until September-October. So, I will put it into the mill [in] September-October. And that will give us yarns by mid-year of next year; and it has to be woven and that takes a few months too, and then it has to be cut... and so it is fabulous getting to see people sort of go, "Oh, fiber, that's an agricultural product." I am like, "Why yes, it is, there are such things as bad seasons and bad crop years, and these sheep were too stressed by the weather and now the wool has wool break, and we cannot use it because your sweater will fall apart and you will be very unhappy when you pay all that money for a sweater and have to return it." (DE 25)

Working more closely with the producers, artisans, craftsman and farmers comes with an entirely different set of considerations and challenges most traditional designers would not encounter. Through operating a sustainable fashion MSE, the design entrepreneurs can have an entirely different relationship with those within their supply chain. Understanding how materials are made and processed influences production, design and business practice decisions.

Aesthetic sustainability considers timelessness, durability and continuous attraction to an object such as a garment (Harper, 2018). A significant majority of designers discussed the characteristics of aesthetic sustainability and how those principles influence their design practice. Emotionally durable design and co-design is a strategy a few designers use to promote aesthetic sustainability and reduce consumption and waste. Designers felt that by working closely with their consumers they could better provide them with the clothes they want. As one designer 
explains, there is more at play than just consumer preferences when it comes to sustainable fashion:

The whole process...is putting much more effort into designing the pieces, so the pieces that actually make it into the collection are very strong. Last week we had a meeting with women who are part of the collective and I sort of made sketches and each person had a copy of the sketch and they made notes about what they like, about each piece, what they would change. And we sat down and we talked about it and we really got deep into the design of these pieces. So, it is about having pieces that are more properly designed and that are more likely to have an impact on the actual consumer, and really catering to the actual individual as opposed to just one store's aesthetic. When people hear sustainable fashion, they assume a certain type of aesthetic, and my aesthetic is not like that at all, so it is hard for me to sort of fit into certain stores. (DE18)

Sustainable fashion design currently involves many trade-offs and limitations. The design entrepreneurs were specifically asked to discuss these. Generally, the designers in this study saw limitations as a positive: treating them as a creative design challenge. Many of the designers embraced these limitations as they found one had to be far more creative to overcome them. This emphasized their commitment to sustainability and skills in resourcefulness and resiliency as the challenges they face are many. It was also clear that aesthetic design was the essential objective and sustainability is the value added, regardless of sustainable strategies used. As expressed by DE21:

It has to be a good design in order to bring people in. I also do not want to be selling people something solely on the fact that it is organic.... They need to wear it; they need to love it; they need to feel good about everything that propels [the] designer... it needs to be desirable.

Material selection is where many of the designers encounter trade-offs. For example, when using deadstock materials or end-of-rolls, designers are frequently using materials with unknown fibre content. For designers where durability of a garment is priority (to reduce the odds of that garment heading to a landfill quickly), the sustainable material option was not always the best, "I guess that is something for me that I really had to decide,... I am not going to compromise quality" (DE21). Depending on the sustainable fashion strategies a designer uses (e.g. zero waste, sourcing within a fibreshed) their practice can vary dramatically. The level of innovation 
and creativity is greater among this group as they navigate obstacles a traditional designer would not face.

\subsubsection{Information}

A series of questions inquired into: whether the designers do research related to sustainable fashion, what were their sources of information, and the potential implications of this. There were also conversations with the design entrepreneurs regarding their knowledge and use of existing sustainable design tools.

For the most part, the design entrepreneurs are self-taught when it comes to sustainable fashion. The great majority of the design entrepreneurs had some sort of formal training in fashion design from post-secondary institutions. Three of the design entrepreneurs attained graduate degrees in sustainable fashion. However, when asked whether they had learned about sustainable fashion in these formal training programs, the majority of designers noted it was minimal to none. Sustainable fashion was not a core aspect of their curricula in design at the time of their studies. Community was cited as the best option as it worked as a source of information about sustainable fashion. This refers to consulting with other designers, colleagues and friends (in co-working spaces, markets, fairs and incubators). There also events, which in cities like London and New York are numerous and frequently enlist expert panels and speakers. The other sources of information identified were online (websites, Instagram).

Few of the designers subscribed to organizations dedicated to sustainable fashion education for MSEs (e.g. the Ethical Fashion Forum, now known as Common Objective). One designer had been a member but did not find that there was enough value for her to continue her membership. Only three designers read or had access to academic papers or current research findings regarding sustainable fashion. Expert 1 felt the information should be more accessible as, "Designers do not have the amount of time that we need to do research - they need to have good quality information available at the click of a button.... A lot of the information right now is proprietary, and it needs to become open-source". DE21 describes her experience researching sustainable fashion: it took "many hours of research; if I was paid for those, I would probably be very rich right now. But it has been sort of trial and error, and a very steep learning curve". A significant majority of designers felt the research associated with sustainable fashion practices is very time-consuming and can be costly. Designer's (DE2) thoughts on the issue: 
[It is] more knowledge - you have to retain so much more information than a conventional fashion designer. I would not have to think about where it was coming from, and how is this happening, and who has been involved in it, and how do I compensate for that, how do I engage the consumer, [or] how do I monitor the feedback. If I just made pretty clothes, then I could just sell pretty clothes.

Academic or institutional research can also be very technical and difficult to grasp. DE12 indicated:

I think it is hard as a non-technical person to feel like they have a complete grasp. Even certifying bodies do not do a great job of communicating what the standards are. I was reading about the Oeko-Tex dye standard... (their white paper on it).... I still do not know how I would go about even introducing that to my dye-house because I would have to be the person to push and begin to explain that to my vendor and I do not see that. I do not envision them doing that research on their own.

Quite a few of the designers noted that they do not spend a lot of time researching as it is too time consuming to keep up with new information, as DE1 noted, "this is what puts people off sustainability. It can be incredibly overwhelming to continuously do all this research and make sense of it."

\subsubsection{Design Tools}

The designers were asked about their awareness and use of such tools. Table 4.3 shows the answers of the designer's responses as to their knowledge of design tools, whether they have used them, their familiarity with underlying concepts of sustainable fashion design tools, and whether they currently monitor progress of sustainability. Part of tool education is teaching designers to define their design problem and determine which tools are relevant. When asked whether they use any sustainable design tools currently, none of the designers indicated that they had done so, although two had done so in the past. 
Table 4.3: Designer's sustainable design tool familiarity and use.

\begin{tabular}{|c|c|c|c|c|}
\hline Designer & $\begin{array}{l}\text { Familiar with any existing tools } \\
\text { for sustainable fashion design? }\end{array}$ & $\begin{array}{l}\text { Have used a tool } \\
\text { for sustainable } \\
\text { fashion design } \\
\text { (impact } \\
\text { assessment)? }\end{array}$ & $\begin{array}{l}\text { Is familiar } \\
\text { with life- } \\
\text { cycle } \\
\text { assessment? }\end{array}$ & $\begin{array}{l}\text { Monitors } \\
\text { environmental or social } \\
\text { impacts in supply chain } \\
\text { or business? }\end{array}$ \\
\hline Expert 1 & Yes & No & Yes & No \\
\hline Expert 4 & Yes & No & Yes & No \\
\hline DE1 & No & No & Yes & No \\
\hline DE2 & Yes & No & Yes & $\begin{array}{l}\text { No - had done a self- } \\
\text { assessment }\end{array}$ \\
\hline DE3 & Yes & Used Nike app & Yes & No - too expensive \\
\hline DE4 & No-interested to know more & No & No & No \\
\hline DE5 & Yes - Nike app & No & No & $\begin{array}{l}\text { No - but would like to, } \\
\text { unsure of how to do }\end{array}$ \\
\hline DE6 & No - interest to know more & No & No & $\begin{array}{l}\text { No - but would like to, too } \\
\text { expensive }\end{array}$ \\
\hline DE7 & Yes & No & No & $\begin{array}{l}\text { No - time is not right, do } \\
\text { not have time/resources }\end{array}$ \\
\hline DE8 & No-interested to know more & No & No & No - not sure how \\
\hline DE9 & No & No & No & No \\
\hline DE10 & No-interested to know more & No & No & No - but would like to \\
\hline DE11 & Yes & No & Yes & $\begin{array}{l}\text { No }- \text { Higg and Nike are too } \\
\text { broad }\end{array}$ \\
\hline DE12 & Yes & No & Yes & $\begin{array}{l}\text { No }- \text { too complex but } \\
\text { would like to }\end{array}$ \\
\hline DE13 & Yes & Yes & Yes & No \\
\hline DE14 & No - seems difficult to use tools & No & No & No \\
\hline DE15 & No & No & No & No \\
\hline DE16 & No & No & Yes & No \\
\hline DE17 & No & No & No & No \\
\hline DE18 & No & No & No & No \\
\hline DE19 & No & No & No & No \\
\hline DE20 & No & No & No & No \\
\hline DE21 & No & No & No & No \\
\hline DE22 & No & No & No & No \\
\hline DE23 & No & No & No & No \\
\hline DE24 & Yes & No & Yes & No \\
\hline DE25 & Yes & No & Yes & No - but would like to \\
\hline DE26 & $\begin{array}{l}\text { Yes - has come across some design } \\
\text { tools }\end{array}$ & No & No & No \\
\hline DE27 & No & No & Yes & No - but would like to \\
\hline DE28 & No & No & No & No \\
\hline DE29 & No & No & No & No \\
\hline DE30 & Yes & No & Yes & No \\
\hline
\end{tabular}

The awareness of tools for sustainable fashion design was also unexpectedly low. Only about a third of designers are familiar with or have heard of sustainable fashion design tools. Nearly half were familiar with the concept of life cycle assessments. None of the designers monitored impacts (either within their business operations or supply chain). Reasons cited regarding why they do not use tools for sustainable fashion design and monitor impacts: 1) they are time-consuming, 2) they are expensive, 3) difficulty in communicating the results, and 4) design of the tools. 
Many of the design entrepreneurs stated there is a burden in using these tools. The time and effort needed to learn and utilize tools is significant and as designers already indicate that sustainable fashion research as too time consuming, this is of no surprise. Many designers felt that time and people were needed to deal with the additional duties that would come with conducting impact assessments, monitoring those impacts and communicating results. One designer indicated, "from what little I know, I would need to have a team who is prepared to put in the work to ascertain the actual carbon footprint of a garment" (DE12).

Cost is another issue as many tools must be purchased, require a membership, or involve a certification process (Kozlowski, Searcy, \& Bardecki, 2019). Those few designers who have familiarity with certifications perceived that for an MSE to become certified (e.g. to the B-Corp standard) or use certified manufacturers or materials (e.g. Global Organic Textile Standard (GOTS), Cradle-to-Cradle (C2C)) was time-consuming and costly. Expert 1 details the issue around the availability of information and solving large social and environmental issues that have broad impacts: "A company has to pay a fair bit of money to get cradle-to-cradle certified. And I understand this is how the world works, but at the same time there is a lot of information that needs to be made public." The point the designer is making is, should access to certain information that can help designers to create sustainable businesses be cost prohibitive? This is where company size can directly affect their ability to purchase, implement, use such tools and/or be certified.

It is clear many of the tools for sustainable fashion design are for large brands with dedicated sustainability teams (Kozlowski, Searcy, \& Bardecki, 2019). MSEs have a disadvantage, as evident through the interviews, as they lack the financial capital and time to invest into learning about and using the tools.

Those who were familiar with specific design tools cited only the the Higg Index and the Nike Maker app. The impressions from these designers was that tools should be simplified to suit the needs of users. This is not to say they did not see the value in these tools, more so that they did not suit how they work. For example, Expert 1 stated,

I do think that they all can be very valuable... in just getting designers to think about things in a new way. With the Higg Index I actually see that more as it is not so much geared towards designers as it is particularly for larger brands.... When the first 
version came out... it was just a bunch of very complicated Excel sheets. I remember opening it and thinking no fashion designer I know will ever use this. They will open it and have the exact same reaction that I did.... Designers spend all of their day taking information in primarily a visual form and I do think there is value in providing information in that form as well to designers.... And also, the risk there is that it reduces sustainability to a bunch of numbers... Those numbers are crucial and important, but they are only a part of the picture.

Indeed, many designers noted they would be unsure how to communicate results to consumers who lack education of tool benefits or use, particularly where assessment tools produce quantified metrics.

DE3, who was familiar with design tools, explained her experience using the Higg Index, "I could not get my head around it to be honest. Maybe the Making App from Nike for me is better because it is a bit more visual and the information is more easily displayed." Tool development and design is a critical factor for tool uptake by fashion designers; tools must be appealing and easy to use from a designer's perspective.

It became evident throughout the discussions that designers are not taught about design tools, nor are they trained on how to use these tools and what the outputs means. How does a designer translate the outputs into meaningful targets, actions or strategies? There is an opportunity in teaching designers about these tools and how to integrate them into their existing design practices. This builds upon Palomo-Lovinski and Hahn (2014) observations that designers are not receiving the training or education to push sustainability forward in an innovative way through a problem-solving scope.

\subsubsection{Branding}

One of the issues explored with the design entrepreneurs was the nature of their branding. Key themes revealed throughout the discussions included: 1) stigma, 2) sustainability as addedvalue, 3) niche categorization, 4) authenticity, and 5) storytelling.

Sustainable fashion has become a "cringy" buzzword and still carries a lot of stigma associated with ugly eco-clothing of the past. While all of the designers within this study offer modern contemporary clothing that does not ascribe to the old "eco-clothing" archetype, the stigma is difficult to shake off. DE21 noted how she is frequently advised to drop "sustainability": "My press feels strongly that I should completely rebrand.” Designers often felt 
it was important not to appear as an ethical/eco-fashion brand, because of the prevailing stigma of eco-clothing as unattractive and unsophisticated. While this is slowly changing, the ecoclothing archetype of shapeless styles, drab muted colour palettes, basic silhouettes, hemps and linens and links to "hippie culture" endures. DE2 had the following to say regarding this stigma, "It is getting over the fact that a lot of people assume that what you do is not either real fashion or not attractive or not worthwhile for them. It is the fact that sustainable fashion is still a different industry to the fashion industry."

The product has to speak for itself first and foremost - sustainability is the added-value. "I try not to pigeon-hole myself as exclusively about sustainability, it is about the product first and it is also about the transparent manufacturing process, who I am working with. Every hang-tag [details] what I am using and who is making it" (DE12).

Designers face the stigma of being criticized and face moral elitism that eco-fashion is simply not cool or aesthetically desirable. Designer's expressed that being labelled as a sustainable fashion brand can still be very niche categorization, preventing them from being accepted and sold alongside mainstream fashion. As DE21 explained:

I actually had a lot of interesting feedback.... A lot of people have told me that I should just be "pure fashion" and the extra additional takeaway should be that it is sustainable... because people are afraid of it right now. It is killing my business a lot too. My PR and Sales team during Market Week do obsess [about the branding]. A lot of big retailers [are there] and some of the feedback I got that was, "Oh, it is really lovely. I would probably take it on if it was not eco because we can't sell eco. We've tried it on our floor before and it does not work because the consumer does not understand it yet." Which sort of kills me. I am pretty sure it was [a major high-end department store].

Several of the designers specified that language used becomes very important as it can put people off and not all consumers care about the sustainability qualities. The most important strategy for branding is authenticity. Authentic storytelling was the most-cited sustainable fashion strategy to market and advertise their brand and products. Designers recognized social media is the most important platform for day-to-day messaging. Instagram is a great tool to highlight daily operations and educate the consumer about production, garment care, sustainability aspects, and values. Branding serves as a promotional tool but also as an 
educational component which is meaningful for an MSE brand to communicate. Sustainable fashion typically has a higher price due to the higher material and production costs. The valueadded sustainability needs to be communicated to bring awareness to those costs. Consumers are dependent on companies for sustainability information regarding products (Delmas and Burbano, 2011; Ritch and Schröder, 2012). The majority of designers felt the best way to create awareness about the values behind their brand was through authentic storytelling. Storytelling is complex because sustainable fashion is complex, and one has to connect the dots for the consumer in an authentic way. Branding can be the most precarious endeavor the designers face.

\subsubsection{Incubators and Accelerators}

One point that was raised was the importance of incubators and accelerators as formal linkages that develop among design entrepreneurs. As Expert 4 explained, "Incubators have always played an important role within the creative community." This reflection of incubators and the support they provide was evident. Among the sample, three designers belonged to a sustainable fashion incubator in New York, two were members of a fashion incubator in Toronto, and two others operated a sustainable fashion incubator and accelerator. In the conversations with the design entrepreneurs, it became clear that those who were involved with incubators and accelerators found it much easier to navigate the challenges than those who did not belong to an incubator or accelerator. Those involved all referred to the ease of having a community for help and support. Sharing was a central theme, as designers remarked the ability to bundle orders to meet minimum order quantities (MOQs). It was easier to find manufacturers or suppliers as a vetting process has generally already occurred. Designers could in fact discuss their experiences with other members about the vendors in their network. As one designer (DE13) described her experience working within the incubator:

Sourcing takes a really long time. I think we are really spoiled having the library here and also just other people to talk to. I would not have gone down the path of doing the piece dyeing for wovens if I did not have [DE12] or I would not have even applied here if I did not know [DE11] from doing those small tradeshows with other designers. So, I think a lot of it is like the community aspect. I can see how it would be really, really hard [not belonging to an incubator]. I think it would be easier if you started somewhere where they have stuff going on like this and then you could move out to a city where there is not so much, but at least you had your original group of people to tap into for resources and stuff. 
Generally, local vendors are known through word of mouth. As one expert-designer noted, "a lot of them do not have websites, they definitely do not have Instagram accounts. So, it is sort of like keeping your eyes out and your ears out and talking to people and listening to who is doing what. It is still a very closed-off industry”. Incubators encourage a more transparent, supportive and collaborative community.

A positive feature of incubators is the environment: the opportunity to share knowledge, reduce costs, and collaborate. The incubators with which the designers were involved both provide mentorship and a variety of resources. For example, one incubator has a sustainable materials library and hosts weekly workshops or events. Designers felt it was beneficial for the local economy when they collectively support local production networks and share sourcing resources such as suppliers or manufacturers. As one designer explains why she is transparent about her supply chain:

On my label, [suppliers and manufacturers are] all printed - their name, where they are located. And the whole idea behind that is to educate my consumers about where it is coming from and hopefully encourage them to ask questions, but also to allow other designers to come and tap into my supply chain and use it for themselves because I am not going to support it myself. So, my vendors appreciate that a lot. (DE11)

One Expert 4 explains the benefits of incubators in supporting local production:

I think it has been playing a very important role on reshoring.... To be able to support and define community, you have to have all the resources. Especially [in] a small emerging creative community, you need to be able to support all of the services that they need.

It is clear from the discussion with the design entrepreneurs, both those working in incubators as well as others, that there are many perceived benefits from developing linkages: mentorship, information sharing, studio space and resources for emerging brands, support for local vendors, craftsmen and manufacturers. They create communities.

\subsubsection{Manufacturing and Sourcing}

Throughout the interviews, design entrepreneurs were asked about their manufacturing practices and the challenges they encounter. Two major themes were identified regarding the 
manufacturing practices of the design entrepreneurs: local production, and managing relationships. The benefits, challenges and implications of the two themes are discussed. This section also includes a description of the related challenges the design entrepreneurs face and discusses how they have managed those challenges.

As noted previously, a high proportion of the designers manufacture locally. Local production has both benefits and drawbacks. Benefits of local production include positive impacts for local economies and communities, transparency, stronger relationships with supply chain stakeholders, greater compliance, and flexibility. Strong local production networks can have a positive impact on the local economy; as DE14 stated, "I just want to make things with the things that are made in the US, and I also believe in investing in the local businesses." The majority of the designers expressed the importance of supporting the local industry to ensure their supply chain stayed in business. It is also considerably easier to maintain a transparent and ethical supply chain when you have proximity. Communication is clear, as in-person meetings can easily take place, and it is easier to build a strong relationship with the vendors in the supply chain. As Expert 6 indicated, "With large supply chains there is a lot of room for miscommunication and error when there is so many hands involved. There is no responsibility and and you have no idea where the issues are coming from and everyone is blaming the guy on the left or the right, everywhere". In situations where certifications are used as part of branding and marketing, compliance is needed or sought. Having suppliers and vendors nearby makes it easier to ensure they are up to date with their compliance. Audits can take place frequently and easily, increasing supplier compliance. As explained by DE12, "I find a lot of my vendors do not necessarily have updated certifications, so I have to push them to actually get them."

Developing a good relationship with manufacturers was recognized as a common theme among the majority of the design entrepreneurs. DE21 described that:

I have a specific relationship with one manufacturer. They know how I work, and they know my standards. That is something every small designer struggles with I think: maintaining that sort of importance with your manufacturer, because they are going to push you off to the side if you only have this very small order and it makes sense for 
them. So, I think developing a strong relationship with a really good manufacturer would be the dream.

As the relationship strengthens, the designers noted manufacturers become more flexible in taking on small orders. Most of the experts stated it is important designers learn how to work with suppliers and manufacturers to build those relationships. Most manufacturers are unwilling to work with new MSEs as they do not want to teach designers about how to produce and work with small orders as Expert 7 explained:

Because the truth is suppliers and manufacturers in the US are jaded. They fought NAFTA. Some of them survived it and some of them have not. They have seen their entire client base move overseas and so there is just a level of trust that is really hard to build. But once you have the tools, the resources and the knowledge of how to build those relationship, you can get further in that door. That is probably the biggest challenge that I would say and is the first one to tackle because it can open up so much.

Regardless of geography, designers saw many types of benefits from working on their supply chain relationships. One of the designers had built a vertically integrated supply chain offshore and had this to say about the education-orientated community work they conduct where their supply chain is located:

What you have the ability to do, in somewhere like Egypt, is if you revive this industry and protect the brand of Egyptian cotton and make it specific and truthful... [is] to train a highly skilled workforce.... Then all of a sudden you have a country where the number of people under the poverty line is way lower; you have more educated people, a lot less illiteracy. And what happens is when you educate people, when they have the ability to go to school economically, and then you educate? Then they understand the benefits and the importance of sustainability and the environment and all that kind of stuff. Like I said, this is a vicious loop where they cannot get out of that. That is why I think economic development is super underrated in the whole sustainability thing. That is a big thing that we are trying to tackle too. Egypt has 100 million people, [it is the] second most populous country in Africa after Nigeria, the biggest in North Africa. It is a place where there is a lot of political turmoil. But when you enable people through education and through economic freedom the stats say there is a lot more peace in places with higher education. I am 
not going to say we are going to create world peace through this. You draw the lines. (DE7)

There also exist a number of drawbacks to using local production, such as higher costs, lack of expertise, and lack of access to certain types of resources. DE12 detailed:

[I] hope that the vendors I work with will start to produce the kinds of stuff that I want or to have access to stuff that is more sustainable. I think about that in terms of zippers. I do not use zippers, or I have not used zippers because [of] the tape for the zipper. You cannot get $100 \%$ organic cotton and the metal—with the exception of Lampo from Italy - people are not using low-lead brass and all that sort of stuff. It is a constant challenge.

Small scale production may not also be the most sustainable option either as the efficiencies of mass production are lost. Expert 6 explained how this occurs:

Producing just a small amount in a mill is not environmentally effective or cost effective. Even though you just want to buy a few yards, it is going to cost you massive amounts. So, just understanding it is not that simple as, "Oh I wish I could get just like a small amount." You will be paying like a massive amount and you will be setting up the looms and dying just a tiny amount of threads.... That is not the answer either. Those are in my mind the wrong questions for some of these designers to be asking. You really need to understand the supply chain. I think a lot of people go into design and go to school and not wanting to do this at all. And if you want to produce sustainably, you have to be a part of it from the beginning to the end: from sourcing the materials to understanding how it is made. It is not just as simple like buying organic cotton and knowing where it is produced.

When asked about these issues, most of the experts indicated that many of the challenges designers face with manufacturing and sourcing could be easily solved by training designers about how to work with the stakeholders in their supply chain to foster better relationships. Expert 4, stated "There has to be more emphasis on supply chains and understanding [them]." She continued that this would be further facilitated if there was support for building infrastructure and assistance for factories to help them adapt to operating in the digital age (e.g. Skype, internet and social media). 
It is clear from the variety of perspectives that have been explored that there are a number of challenges that designers faced with manufacturing and sourcing (Table 4.4). Key challenges will be discussed.

Table 4.4: Manufacturing and sourcing challenges faced by MSE design entrepreneurs

\begin{tabular}{l}
\hline Challenges \\
\hline Minimum order quantities (MOQs) \\
\hline Higher cost of ethical labour and small production runs leads to lower margins and/or higher price points \\
\hline Higher cost of sustainable materials leads to lower margins and/or higher price points \\
\hline Scalability \\
\hline Competition with fast fashion pricing
\end{tabular}

The design entrepreneurs face all the same challenges that a traditional small business would, but they are magnified in numerous ways. The first and foremost frustration, as cited by every designer, is the MOQs imposed by suppliers and manufacturers. The additional burdens these design entrepreneurs face are the vetting process of suppliers, mills, materials and manufacturers to ensure compliance and ethical practices, maintain transparency, and/or verify certifications. There is also considerably less choice and range of materials, manufacturers, suppliers and technologies available. A designer (DE20) describes how limitations with materials impact her, "I have to choose from the sustainable fabrics that [are available] in order to create something cool that I like, and this is also another effort in such a big-time investment as well, for me, because sometimes I just wish I could use any fabrics and my life would be so much easier." Sustainable materials, process and technologies are also far costlier and can be more time consuming. Many designers stated having to "compromise a lot which really annoys me.... For example, the last collection I designed, I chose an amazing [sustainable] fabric... and was told after I already did the designs based on those fabrics that I could not have some of them unless I commit to their units... That makes me angry as a designer because the end product is not how I wanted it to be. I just have to compromise" (DE3). Another designer (DE21) also noted that the sustainable option is not always the most durable; for example, materials could be of lesser quality.

The majority of the designers indicated that high MOQs make it difficult for designers to commit to buy as most orders start around 1000 yards (900 metres). This also makes it difficult for designers to purchase sampling lengths before production and find space to store such large quantities. This is complicated since most designers tend to store scrap material in an effort to 
reduce their waste. A common approach to mitigate MOQs is partnering with other designers on fabric purchases, asking for in-stock options which do not normally have as high MOQs, or piggybacking onto bigger orders by larger brands. This of course is contingent on designers agreeing to purchase the same materials. There are limited choices when using in-stock options or piggybacking. Developing a relationship with a supplier may lead to reductions in MOQs but this of course takes time and is typically associated with a surcharge. Also, as mentioned previously, sustainable designers are looking to reducing waste and produce small runs, so overbuying on materials to meet MOQs is not ideal system within which to work. Designers who work with deadstock struggle with not being able to reorder materials and having to work within the set quantities of what is available.

For MSEs, fabric consideration is significant for a variety reasons as explained. Many of the designers therefore chose to design with a limited number of fabrics in a collection. This helps to reduce waste. It is easier for production when dealing with limited fabrics and could help with manageable MOQs and costs. However, when it came to working with suppliers to improve their processes, designers struggled as they found they do not have influence as a small company and the same challenges re-appear: "We are looking at someone that uses organic plant-based glue for fusible but we would have to use 3000 yards of it in order for them to agree to it" (DE 12). This stems from a larger problem in that shifting to sustainable practices is costly and time consuming for suppliers and manufacturers as well. This is especially true for certified materials and processes as one designer explains the implications, "The entire chain of custody would have to be certified and that is super onerous for smaller growers, spinners, processors that are not vertically integrated, so I think it is a trade-off. I am pushing for stuff where I can and realizing and communicating of the realities of what we are meeting but not being able to show certification to the customer" (DE12).

The majority of designers discussed pricing and costs as another key challenge. Producing sustainably using sustainable materials is more expensive. Small production runs are also more expensive because a designer cannot take advantage of economies of scale. And the problem is the consumer does not understand why sustainable fashion is more expensive. As Expert 4 affirmed:

If you went out and said, "Do you know how many chemicals might have been used in the processing of the garment that you are wearing right now? Do you any idea what effect on your health those chemicals might have? What happens to 
them when you discard that garment because it is thrown away and it is put in the landfill? Do you know the price the environment is paying? Do you know how the people were treated or mistreated, that produced that piece of clothing? Do you realize you may have paid for child labour or for sexual abuse or unsafe unhealthy working conditions, that someone may have died producing that piece of clothing?" Do you think the average person has any concept of that? Because I do not.

Designers have found ways to remedy consumer education, but it is slow and not widespread. Many mentioned they try to educate their consumer through Instagram by detailing the story of how their products are made or providing very detailed hand tags with full transparent information regarding the supply chain and materials. Designers also noted that they reduce their margins in order to offer better prices to their consumer and remain competitive. As a designer notes, "The problem is that I think I sell less because of the price, but this is another thing, where if people understand what they are paying for" (DE20). Another, DE3, explains her decision to produce in offshore to maintain a competitive price point, "If I was to do production in London the prices would be triple of what they are. Fabrics are three times more expensive.... It is crazy. If you want to do 'Made in U.K.' and you use U.K. fabrics, you have to go high-end. There is no way you can have a High Street brand and do everything in the U.K."

The cost of running a sustainable fashion MSE is considerably more than a traditional MSE fashion brand. However, the overall majority of design entrepreneurs do not wish to become a large global brand defined by the success of its profit margins; their goals are more modest. Instead these design entrepreneurs define success for themselves in a different manner where profits are not the key measure, as DE21 noted:

I do not make a lot of money, but I do not do it for that. It is going to be a long time before I make money off of it. It is a real passion project. It covers my wholesale markup.... I am not doing too much mark-up at all; it probably covers costs, and maybe [a mark-up of 1.3] or something like that, which is okay. It is not ideal for how much I am selling... but it is okay. I am happy with myself.

Another designer (DE12) stated, "I do not think growth for growth's sake is worth it. There has to a purpose of something and I would like to get to the point where I am influencing the industry in a meaningful way." 
However, as some of the designers who have been in business for over ten years noted, the situation for sustainable fashion design entrepreneurs has improved. There are more options available for sustainable materials and notions, more resources available in the form of incubators, accelerators and organizational groups, a growing collaborative community, and more manufacturers catering to smaller production runs.

\subsection{Discussion}

A number of themes and observations can be drawn from the interviews with the designers and experts. The designers are interesting people who are inspirational, creative, innovative, committed to their ideals, and responsive to the issues. They have very clear visions of how they want to work and see themselves as leading a movement. These design entrepreneurs wish to reconcile personal fulfillment with professional achievement as they seek to flourish sustainability within fashion by competing against the dominant paradigm of fast fashion. The conversations revealed numerous benefits and opportunities to owning and operating a sustainable fashion MSE as well the types of challenges the design entrepreneurs confront. Local and fair ethical production were dominant approaches favored by the MSEs for a variety of reasons. A significant majority of designers recognize waste and overconsumption as one of the most critical negative impacts of the fashion industry that need to be addressed. There is a consensus among the designers and experts of a need to shift away from the term "sustainable fashion" in favor of more explicit language describing a designer's unique perspective on sustainability. Collaboration, balance and culture are also identified as fundamental drivers for the design entrepreneurs.

As demonstrated through the interviews, operating outside of the traditional fashion system provides the design entrepreneurs with the space for creativity, flexibility, collaborations, innovation and control over their brand. It is argued it also creates challenges with respect to sourcing, stigma, a niche categorization, financing, greater costs (lower margins), customer acquisition, marketing exposure, and competition with pricing (fast fashion). The research findings support conclusions drawn by Lawless \& Medvedev (2016) that current economic models do not support the development and adoption of sustainable fashion practices. This research also provides further evidence of the enormous financial potential and opportunities for MSEs to drive sustainability (Battaglia et al., 2014; DeLong et al., 2016; Lawless \& Medvedev, 2016) despite the identified barriers. It was evident that the flexibility, creativity and 
commitment to the beliefs exhibited by the design entrepreneurs are instrumental in navigating the many challenges that come with running a sustainable fashion business.

Local production networks provide MSEs with the flexibility and control of their supply chains that facilitate their sustainable business and anti-fashion calendar models (e.g. made to order) and transparency. Local production networks operate in defined geographic localities, have a strong socio-cultural element and include a high proportion of small-to-medium sized enterprises, influencing their abilities and future prospects (Dimitriadis \& Koh, 2005). These local production networks allow design entrepreneurs the space for creativity, collaboration and innovation by enabling them control over all aspects of their design and business practices and supporting their sustainability initiatives. The close proximity of their supply chain supported the designer's ability to ensure transparency of fair and ethical labour. This also enabled the design entrepreneurs to engage in sustainable business models as many chose to produce on demand D2C as a means of reducing waste through overproduction, especially when working with such tight margins. On-demand production necessitates close proximity of production facilities.

A consensus among most of the designers is that waste and overconsumption are one of biggest issues. Many feel conflicted as to whether they are providing a solution or contributing to the problem, as they still cannot run their business in their ideal way due to the limitations in production, technology, system structure and materials available to them. This reinforces that sustainable fashion and sustainable fashion design are very complex, and the trade-offs are not easy to negotiate. However, many positive opportunities were seen. The community is very inviting and relatively open source-designers are willing to share their information, knowledge, suppliers, factories, manufacturing in an effort to improve the industry as a whole. There is a shift towards an anti-fashion calendar model as evident by the practices of many of design entrepreneurs in this study. This has many benefits for designers as it is very stressful to produce at the rate of the current calendar which leads to overconsumption and waste. These MSEs are able to produce on an as-needed basis. This is achieved by primarily using local production which allows for in-season production, allowing for better predictions of what the consumer will want. Social media has proved to be a great tool for these designers to engage with their market online and solicit preferences for styles and colours.

Collaboration is also vital for developing sustainable fashion and mitigating the challenges that these MSEs face. For more cross-industry collaboration is needed-among and between brands and suppliers. Sustainability is a culture and a lifestyle and that is what the design 
entrepreneurs' practice and aim to grow: a culture of sustainability. These designers are not looking to be millionaires or celebrities; it is about creating a balanced lifestyle that celebrate their love for fashion in a fair and ethical way that does not harm people and the planet.

The consensus among the designers and experts is that a new term or shift away from the term "sustainable fashion" is needed. There is a stigma that still exists and as sustainable fashion MSEs, these designers must face higher costs for producing sustainably. They need to navigate the tradeoffs of branding while educating their consumer to understand these higher costs. The sentiment is that sustainable fashion is overused and has become a broad buzzword. A shift to explicitly stating how a design entrepreneur operates, such as zero waste, ethical or fibreshed sourcing, is more beneficial for MSEs.

\subsection{Conclusion}

This study investigated and presented a case study of how sustainable fashion designer entrepreneurs who own and operate an MSE integrate and foster sustainable practices into their design process. It has explored the entrepreneurial motivations, knowledge and use of sustainable practices, business practices, design process, and branding strategies. These designers are driven by personal beliefs and values that are manifested through their entrepreneurial activities and design practices.

Common identified strategies used by the design entrepreneurs include zero waste pattern drafting and production, sustainable material selection, designing for aesthetic sustainability, ethical and local production and alternative business models. We demonstrate that sustainable fashion MSEs have an innovative approach to sustainability characterized through their use of an anti-fashion calendar and D2C business model, transparency to assure fair and ethical garment production, collaboration, a zealous commitment to reduce waste and a revalorization of craftsmanship and working with artisans. The design entrepreneurs who design and produce locally do so in order to exert greater control over their supply chain. Many of the participants operated outside of the traditional fashion system which provides the design entrepreneurs with the space for creativity, flexibility, collaborations, innovation and control over their brand and business. This study contributes new insights and understanding into the design practices and entrepreneurial activities of sustainable fashion MSE. It also contributes to the emerging studies of fashion design entrepreneurs and their desire to stay local and craftsmanship promoting high quality garments thereby altering perceptions of how we value clothing. 
Implications of these designers choosing to produce locally is a prediction in the rise of small-scale manufacturing and the creation of local production networks in contemporary cities. This could be true for fashion capitals, such as New York or London, but also cities not necessarily known as "fashion capitals", such as Chicago, Detroit, Toronto, or Montreal. A key finding of this research is the lack of a clear definition of what sustainable fashion is or the associated activities. Instead, sustainable fashion as demonstrated by the MSEs includes a wide range of activities, opportunities and possibilities. MSEs also face particular challenges with respect to sourcing, stigma, a niche categorization, financing, greater costs (lower margins), customer acquisition, market access, marketing exposure, and competition with pricing (fast fashion). Overall, MSEs have been proactive in integrating sustainability into their businesses, however they need further support with the resources, systems and structures to succeed in their endeavors for a sustainable fashion system.

Future research could therefore focus on the challenges faced by sustainable fashion MSEs and how to support local production networks to build collaborative circular economies locally. MSEs are a positive contribution to the sustainable fashion space but need support with the resources, systems and structures to succeed in their endeavors for a sustainable fashion system. This includes supporting local production network activities and fostering collaboration with all stakeholders. It is important to note support should also extend to diversifying the sustainable fashion space. There is not much diversity found among sustainable fashion brands in general and among those design entrepreneurs and experts who participated in this study, most of whom were predominately white women. Racial and gender diversity must be supported to achieve a truly sustainable industry (Kozlowski et al., 2019). Many of the barriers, benefits and opportunities can be identified, solutions to barriers can be created and opportunities expanded. As Expert 4, a pioneer in the field of sustainable fashion, confidently expressed, "I actually think it is the small emerging designers that are the real future of our economy... [which is] likely to be a collaborative sort of network of emerging designers, of artisans, of brand, of partners, of all sorts of things." 


\title{
5. THE reDESIGN CANVAS: FASHION DESIGN AS A TOOL FOR SUSTAINABILITY
}

\begin{abstract}
Many of the existing tools for design in a sustainable fashion context are too complex, overly conceptual, require experts to apply, have a high cost, were created for large corporations, or fall short in holistically supporting sustainable fashion design entrepreneurial practices. Micro-sized enterprises represent a significant portion of the fashion industry and can meaningfully contribute to the transition to a more sustainable apparel and textile industry. This paper addresses this gap through the development of an original design tool, the reDesign canvas, to support design entrepreneurs in developing sustainable fashion enterprises. Informed by design thinking and systems thinking, the canvas was developed based on an in-depth review of the academic literature and the collection of qualitative data. Qualitative data were gathered through both participatory action research (PAR) and interviews with 37 sustainable fashion design entrepreneurs and experts in sustainable fashion. Both the PAR and the interviews were used to test and refine the reDesign canvas in order to ensure it meets the needs of sustainable design entrepreneurs operating micro-sized companies. The final version of the canvas is based on 12 building blocks that a design entrepreneur would encounter in building a sustainable fashion brand. The reDesign canvas can help advance both the theory and practice of sustainable fashion design.
\end{abstract}

\subsection{Introduction}

The production, consumption and disposal of apparel products has a number of negative environmental and social impacts. These impacts are expected to grow substantially in the coming years. Global Fashion Agenda and Boston Consulting Group (2017) "project that the overall apparel consumption will rise by 63\%, from 62 million tons today to 102 million tons in 2030 - an equivalent of more than 500 billion T-shirts" (p. 9). As it stands currently, only 20\% of clothing is collected for reuse or recycling, with the vast majority eventually ending up in landfills ((Global Fashion Agenda \& Boston Consulting Group, 2017).

Responses to the wide-scale negative environmental and social impacts of the fashion industry have been reactive and focused on piecemeal strategies that lack a cohesive perspective. 
These responses have also focused predominately on technological and cost aspects related to the production of apparel. Products are designed and produced for rapid trend turnovers through obsolescence and premature disposal, thereby enabling quick profits - rather than rethinking how design and manufacturing can incorporate consumer needs and sustainability (Niinimäki \& Hassi, 2011). However, the idea of what sustainable fashion is and how it fits within the industry continues to evolve. This translates into an assortment of visions, ideas, processes and products associated with sustainable fashion.

There is no generally accepted definition of sustainable fashion. Most definitions of sustainable business activity directly build on the definition of sustainable development provided in the Brundtland report (Our Common Future) from 1987: "development that meets the needs of the present without compromising the ability of future generations to meet their own needs" (WCED, 1987, pg. 43). The report also identifies three dimensions that form the basis of sustainability: economic, environmental and social. Many researchers, particularly proponents of the "triple bottom line" (Elkington, 1997), interpret sustainability as requiring equal consideration and integration of those three dimensions in the decision-making process. Others, particularly proponents of the embedded view (Marcus, Kurucz, \& Colbert, 2010) argue that the dimensions form a hierarchy, where the economy is seen as part of larger society, which, in turn, is nested within the natural environment. In either case, a key emphasis is the need for any firm to ensure the ethical and responsible management of natural resources and workers throughout both its own internal operations and its supply chain.

Sustainable fashion is expressed in many forms yet lacks any agreed-upon formal definition. A review of literature finds little in the way of a definition; rather, characteristics, ideals, processes and philosophies drive the discourse. For the purposes of this paper, sustainable fashion is conceptualized as fashion that is created taking into account all phases of the cycle of clothing and realizing a holistic and systems thinking design perspective that reduces negative impacts and seeks to create positive economic, environmental and social impacts. Sustainable fashion involves good practices that aim to enrich and have a positive impact on communities, cultures, societies, people, other organisms and the environment, to respect and encourage diversity. Success and profits are decoupled from growth with the goal of creating a system which can be supported indefinitely where environmental and social responsibility drive new economic and business models, ideals and practices. For the purposes of this paper, sustainable fashion is therefore defined as "the profitable design, production, distribution, and end-of-life 
reuse, recycling, or disposal of fashion that supports circular systems, minimizes negative and maximizes positive impacts on both society and the natural environment".

Sustainable fashion is closely connected to innovation. Boons, Montalvo, Quist, and Wagner (2013) view innovation in a sustainable context as radically redefining and moving away from incremental (product and process) to transformational (services, future orientated) innovations that do not cause environmental or social harm. von Hippel (2017) introduces the idea of free innovation, which complements the aims of sustainable fashion and he defines free innovation "as a functionally novel product, service or process that (1) was developed by consumers at private cost during their unpaid discretionary time...(2) is not protected by its developers, and so is potentially acquirable by anyone without payment-for free" (p.1). For the purposes of this paper, sustainable fashion innovation is defined as "transformational changes to fashion products and services developed in consultation with consumers" which advance "the profitable design, production, distribution, and end-of-life reuse, recycling, or disposal of fashion that supports circular systems, minimizes negative and maximizes positive impacts on both society and the natural environment".

Several authors have argued that designers, design thinking and a systems thinking perspective are essential to developing innovative solutions for sustainability in the fashion industry (Armstrong \& LeHew, 2011; Fletcher, 2008, 2010; Fletcher et al., 2012; Hethorn \& Ulasewicz, 2008; Niinimäki \& Hassi, 2011). Sustainability must be embedded in the design process if the industry is to reduce its adverse impacts in a meaningful way. Unfortunately, fashion designers generally lack the tools to enable design-driven change.

Tools are urgently needed to facilitate the transition to a sustainable fashion system. This paper presents a design tool, in the form of the reDesign canvas, to aid designers in building sustainable fashion brands. The tool is particularly targeted to the needs of design entrepreneurs running micro-sized enterprises (less than 10 employees). Design entrepreneurs represent a rapidly growing segment of sustainable fashion brands, but often approach the integration of sustainability into their brands in an ad hoc, unsystematic manner. Design entrepreneurs require a flexible tool that recognizes the different starting points, competitive environments, and resource constraints they face. The reDesign canvas was extensively tested through a participatory action research (PAR) study with a micro-sized enterprise and interviews with 38 entrepreneurs and experts in sustainable fashion. 
The reDesign canvas is a visual tool that resembles a painter's canvas, preformatted with a set of building blocks which form the basis of the canvas (i.e., the tool). The goal of the reDesign canvas is to facilitate a transition to sustainable fashion. Specifically, the canvas allows design entrepreneurs to fully visualize all of the components of new or existing sustainable fashion brands. The reDesign canvas is intended to be printed on large scale surfaces (e.g., a poster) so design entrepreneurs can sketch and discuss the elements of a sustainable fashion brand using markers and/or attached notes. The building blocks are the fundamental elements a designer should include in building a sustainable fashion brand. Fostering understanding of how the building blocks interrelate, the reDesign canvas encourages discussion, creativity and analysis so designers may create clothing that engages consumers through new business models, creating new values, meanings and behaviours.

The reDesign canvas challenges design entrepreneurs to move beyond traditional product design to fully integrate sustainability into their design processes. The canvas makes several contributions to research and practice. As a strategic tool, the canvas facilitates an iterative process where design entrepreneurs work through a series of building blocks, including concept, design and materials, (de)branding, innovative and sustainable business models, consumer engagement, circular design and economies, prototypes and product development, sustainable supply-chain, data management, sourcing, revenues and cost, and stakeholders. Recognizing the need for flexibility, the canvas has been developed to allow design entrepreneurs to work within any, some, or all the building blocks.

\subsection{Theory and Background}

This section introduces the concepts of design thinking and systems thinking. The use of theory and literature form the conceptual basis for the development of the reDesign canvas and the practice-based testing and refinement of the tool.

\subsubsection{Design Thinking}

Design traditionally has been tasked with the making of products; more recently, the discourse has increasingly highlighted the application of design thinking to larger scale social and system solutions (Brown \& Wyatt, 2010; Hethorn \& Ulasewicz, 2008). Design thinking can be described as a "practice... associated with having a human-centered approach to problem solving in contrast to being technology- or organization-centered. [Designers] are seen as using an iterative process that moves from generating insights about end users, to 
idea generation and testing, to implementation" (Kimbell, 2011). Design thinking is frequently presented as an approach to foster innovation while remaining a flexible, expansive and explorative activity (Brown, 2008; Buchanan, 1992; Dorst, 2011; Norman, 2013). For the purposes of the paper, the researchers adopt the understanding of design thinking tied to Brown (2008) and Dorst (2011). The central insight is that design does not necessarily follow an orderly process. Rather, it is the approach to design and the principles underlying that approach that are critical. In any case, it is essential that values, such as a commitment to sustainability, are embedded early in the design process.

Fashion offers and generates multiple opportunities to apply sustainable concepts and practices (Hethorn \& Ulasewicz, 2008) and designers often want to create more sustainable garments but they need to know how to do so. Design is the point of greatest potential in reducing the negative environmental and social impacts of fashion products Fletcher et al., 2012; Niinimäki \& Hassi, 2011; Vezzoli \& Manzini, 2009). Many of the choices designers make encode products for a specific destiny, resulting in a domino effect as they drive the actions and choices of partners and stakeholders from production to retailers, end-of-life and disposal (Armstrong \& LeHew, 2011; Guidice, La Rose, \& Risitano, 2006; Wimmer, Züst, \& Lee, 2004). Thus, "if designers are perceptive and open to sustainability principles, fashion could prove a powerful conduit in the transition to environmentally friendly and socially responsible production and consumption, attracting attention, energy, and imagination around sustainable solutions" (Armstrong \& LeHew, 2011,pg. 31). The reDesign canvas is offered with this point in mind. The canvas must therefore:

Proposition 1: Ensure that sustainability is explicitly integrated into fashion design processes and practices.

\subsubsection{Systems Thinking}

Systems thinking is gaining support in tackling problems for the development of sustainable fashion (Fletcher et al., 2012; Georgiou, 2007; Niinimäki \& Hassi, 2011; Porter \& Córdoba, 2009). Fashion is a system that can be analyzed and is composed of stakeholders, practices, structures, institutions, organizations and behaviours that dictate the processes through which the industry functions. Systems thinking uses tools and concepts to develop an understanding of the interdependent structures of dynamic systems (Meadows, 2008). When designers have a better 
understanding of the fashion system, they are better able to identify the leverage points that lead to desired outcomes or aspired value (Meadows, 1999).

Sustainable business models and consumer engagement are among the key leverage points that can enact positive change towards developing a sustainable fashion system. For example, systems thinking can encourage a design approach that extends beyond a user-centered focus to include all stakeholders within the fashion system. Systems thinking can also encourage designers to move beyond traditional design and product development processes that encourage a take-make-dispose mindset and economy (May-Plumlee \& Little, 1998).

Designers must realize that open, complex problems and opportunities, such as innovative sustainable design in small enterprises, is part of a larger whole. Solutions are likely to require understanding of the entire system (Norman, 2010) to evaluate aspired value, working principles and frames (Dorst, 2011). With that in mind, the redesign canvas must:

Proposition 2: Consider how the design impacts the larger fashion system, including production and consumption processes.

Proposition 3: Recognize the linkages and interactions of the fundamental components within the fashion system.

\subsubsection{Sustainability and the Business of Fashion}

Small and medium-sized enterprises (SMEs) represent a large and often ignored segment of the fashion industry. SMEs are often new ventures operating in niche markets where sustainability is the core of their mission and business model. Micro-sized sustainable fashion companies provide an example of such ventures. Characterized by an entrepreneurial style that favors a lean organization structure (Bos-Brouwers, 2010), SMEs can be strongly value-driven (Jenkins, 2009). This puts SMEs in a much better position to innovate radically and compete successfully in niche markets compared to much larger companies (Schaltegger \& Wagner, 2011). There is increasing recognition regarding the ability of entrepreneurship and SMEs as a conduit for change to sustainable products and processes (Hall, Daneke, \& Lenox, 2010). To this, however, they must adopt a sustainable business model. 
A business model fundamentally defines how an organization operates. It provides the rationale through which the business creates, delivers and captures value. Value propositions are "based on bundles of products and services that create value for a customer segment" (Osterwalder, Pigneur, Bernarda, \& Smith, 2014, pg. xvi). Business models are also viewed as an important driver of innovation (Osterwalder \& Pigneur, 2010). Lüdeke-Freund (2010) defines a sustainable business model (SBM) as "a business model that creates competitive advantage through superior customer value and contributes to a sustainable development of the company and society" (p. 17). However, while new approaches are emerging (e.g. Boons \& LüdekeFreund, 2013); a review of the literature finds no consensus on how a business should approach embedding sustainability (Bocken, Short, Rana, \& Evans, 2014; Boons \& Lüdeke-Freund, 2013).

Business model innovation offers new approaches in rethinking perceptions of value, particularly for new companies or product lines (Bocken, Short, et al., 2014; Stubbs \& Cocklin, 2008). This includes not only value propositions to customers, but also the role suppliers play in creating value (Frank Boons \& Lüdeke-Freund, 2013). Collaboration is frequently cited within the literature as an approach firms need to consider in developing sustainability (Bocken, Short, et al., 2014; Frank Boons \& Lüdeke-Freund, 2013; Lüdeke-Freund, 2010). This is particularly important for SMEs, as they may not have the resources to address sustainability issues on their own. This also further underlines the need for a systems perspective. It is therefore not surprising to see that the literature on sustainable business models is increasingly being linked to the concept of circular economies (CE) and closed loop products.

The ultimate goal of CE is to decouple environmental pressure due to resource use from economic growth. In the transition to $\mathrm{CE}$, a fundamental requirement is the involvement of all stakeholders and their capability to link and create suitable collaborations and exchange patterns (Bocken, de Pauw, Bakker, \& van der Grinten, 2016; Ghisellini, Cialani, \& Ulgiati, 2016). Design plays a critical role in this. Integrating CE concerns must be done within the early stages of product design (e.g., the ideation and inspiration phases within design thinking). Change is difficult once the resources and activities have been committed to a certain product design (Bocken, Farracho, Bosworth, \& Kemp, 2014). With the above in mind, the reDesign canvas must: 
Proposition 4: Recognize that the concept of circular economies underlines the fundamental components of the fashion system.

\subsubsection{Strategies and Tools for Sustainability}

While awareness and research into sustainable fashion has grown significantly in the last decade, the question of how to integrate the concept of sustainability into the fashion design process remains. Recently, there has been an emergence of strategies and tools aimed at the designer in the pursuit of sustainability. For example, Niinimäki and Hassi (2011) discuss design strategies for extending the life span of clothing products while Laitala, Boks, and Klepp (2015) explore the user centered design methods to delay clothing disposal, highlighting the crucial aspect of consumer behaviour considerations throughout the design process. Armstrong and LeHew (2011) provide a review of sustainable design approaches and models used within other industries. Their findings indicate two primary components for a transition to a sustainable paradigm: tools are needed for decision making, and advancement for a sustainable supply chain includes costing strategies, collaboration and cooperation. Both are relevant for the development of the reDesign canvas, with particular emphasis on the first component.

Aakko and Koskennurmi-Sivonen (2013) provide one of the few models developed specifically for fashion designers who wish to incorporate sustainable considerations into their design process. The model is theoretical and focuses primarily on the production process. Another model utilizing the cradle to cradle approach was created by Jin Gam et al. (2009), which also primarily focuses on the production process with little discussion of consumption behaviours, use or disposal. The Ted's Ten (Textile Environment Design, 2006) is a set of ten cards, each having a written strategy that includes a short description and a few examples. These studies and tools have provided much needed guidance for designers, but do not stress the need to explicitly consider the business model underlying a fashion company's activities from which supply chain and product development decisions are derived. Presently, most of the approaches consider sustainable design as a journey rather than a destination (Walker, 2006) and approaches and strategies to sustainable design and product development remain in their infancy (Armstrong \& LeHew, 2011).

Design activities not only influence the production process (e.g., material selection, energy/water use, processes, and manufacturing), but also the consumption process, which 
includes how consumers buy, use and dispose of clothing. Addressing consumption issues requires designers to consider lifestyles and behaviours and their effect on consumption patterns. Design represents a key inception point for many impacts and is considered as one of the most powerful tools for transforming these new roles, patterns of behaviour and opportunities to lead a cultural and systematic change within fashion (Armstrong \& LeHew, 2011; Fletcher et al., 2012). While it is widely acknowledged that sustainability is imperative for the future of the fashion industry and the planet, it is less clear what the means for achieving a sustainable fashion industry are (Aakko \& Koskennurmi-Sivonen, 2013; Black, 2012; Fletcher, 2008). A sustainable design model can be useful in achieving a fundamental understanding of sustainable fashion design and the product development process, but it is clear that any such model must be flexible enough to accommodate the wide variety of needs and tools associated with fashion design. The reDesign canvas is offered with this in mind and must:

Proposition 5: Ensure the reDesign canvas is suitable to the unique needs of design entrepreneurs.

Proposition 6: Recognize the need for flexibility to accommodate differing design needs and approaches to address those needs.

The propositions outlined in this section served as a checklist throughout the research process and development of the reDesign canvas.

\subsection{Methods}

The development of the reDesign canvas was an iterative process that took place between 2014 and 2016. It involved two stages: (1) the conceptual development of an initial canvas informed by the academic literature and the propositions articulated in the previous section; and (2) a revised version informed by data gathered through fieldwork in the form of in-depth semistructured interviews, field observations, and participatory action research (PAR). Figure 5.1 summarizes the research design framework. 


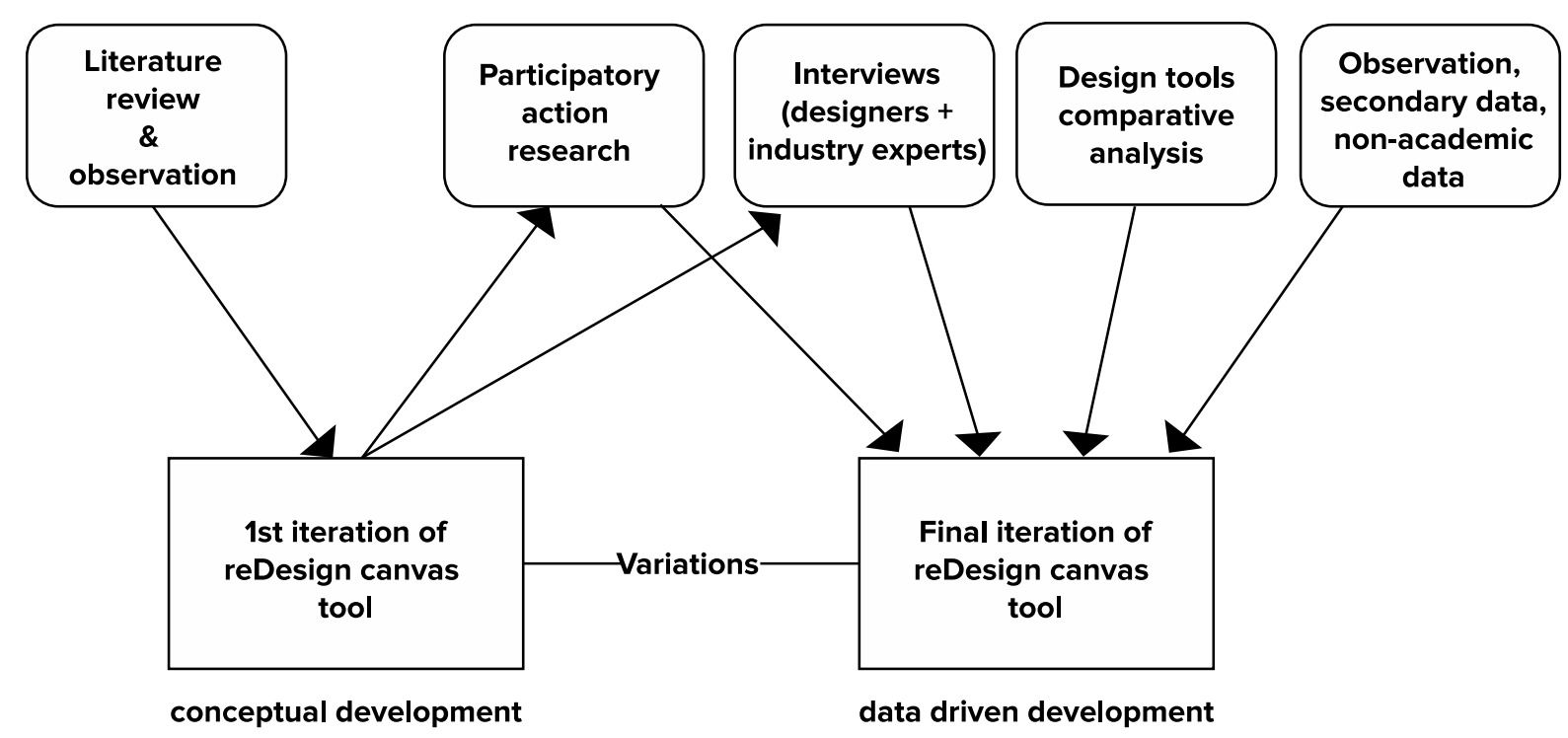

Figure 5.1: Research design framework

\subsubsection{Conceptual Development of Initial reDesign Canvas}

The conceptual development of the reDesign canvas began with a review of the literature. This resulted in the identification of a key set of themes, which formed the basis for the propositions outlining the requirements the canvas must fulfill. This, in turn, provided the basis for the initial building blocks. As a tool for design entrepreneurs, it was important that the format of the tool resonated with how designers work. The canvas framework, an accessible visual form, was adopted from Alex Osterwalder's business model canvas which provides a visualization of all the building blocks involved in an operating business (Osterwalder \& Pigneur, 2010). Each building block describes the most important factors for that block and includes questions design entrepreneurs should address. The key content in the canvas is structured within building blocks which are easily identified by the user, are visually encompassed in a singular space, and encourage a non-linear, iterative design thinking practice. Systems thinking provided the basis for highlighting the relationship and interactions among the building blocks. The initial building blocks were: material selection, sourcing, sustainable business model innovation, sustainable supply chains and production, sustainable consumer behaviour, and stakeholder analysis. The initial canvas was then tested through PAR. The initial reDesign canvas was designed to provide flexibility for individual variations and iterations. 


\subsubsection{Participatory Action Research}

The first key test of the draft reDesign canvas was through a PAR conducted with a sustainable fashion entrepreneur. PAR explicitly focuses on systematic, joint problem-solving and is, thus, well-suited to practitioner-based/applied research (Walter, 2009). The PAR was a collaboration between the principal researcher and the entrepreneur/owner and the project manager of a textile-focused design incubator. As a method where the researcher has an active role, PAR is an iterative process of planning, action, and observation (Walter, 2009). PAR was chosen to ensure that the reDesign canvas included the complexities of real-world design practice. Figure 5.2 demonstrates the iterative and systematic approach used in the PAR conducted in this study (Walter, 2009).

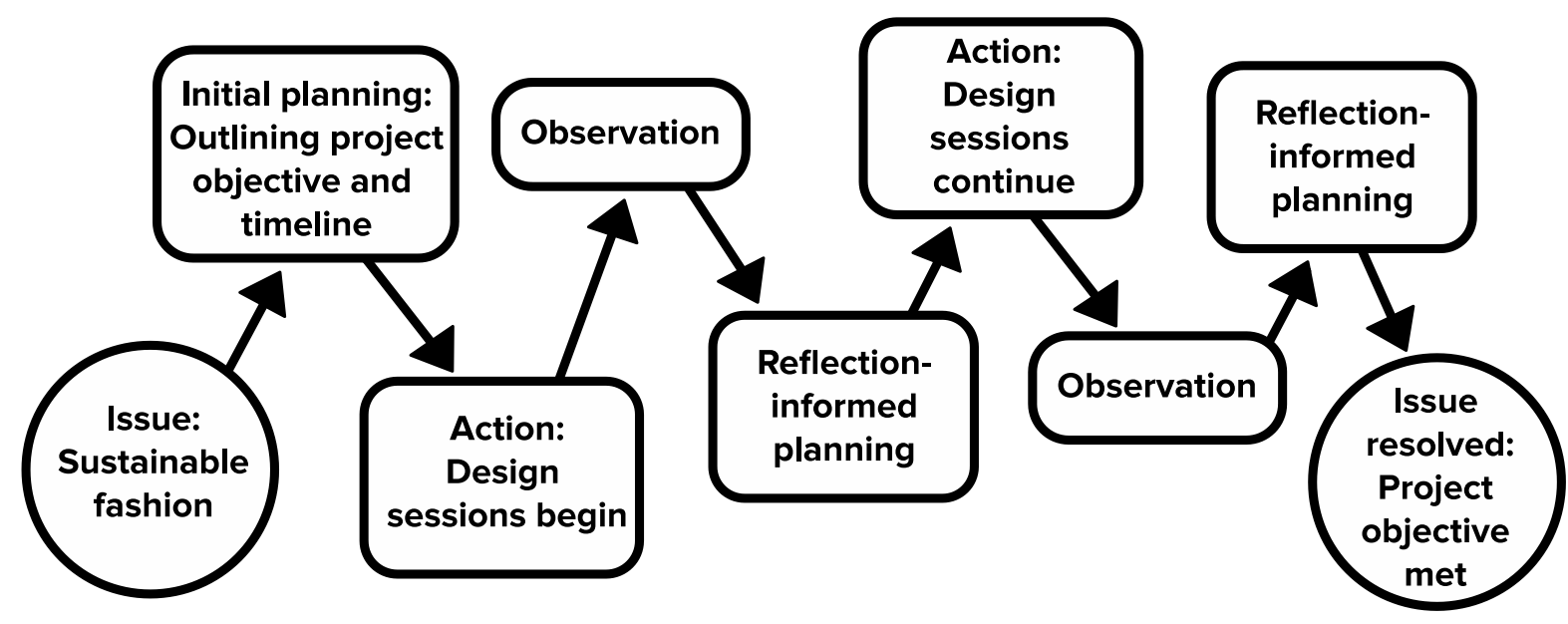

Figure 5.2: The iterative cycle of PAR (adapted from Walter, 2009)

The initial idea for the sustainable fashion product, a women's tote bag, was put forth by the entrepreneur. The principal researcher, a trained fashion designer with industry experience, occupied the role of designer in the PAR.

The first draft of the reDesign canvas was applied and tested throughout the PAR, working collaboratively with the entrepreneur. Using the reDesign canvas as a basis for shared thinking and discussion, the tote bag was designed along with a sustainable business model that focused on circular design and sustainable consumption behaviours. The PAR then proceeded through an iterative process of planning, action, and observation throughout a series of design sessions with the entrepreneur and manager. This iterative process resulted in changes to the bag through multiple prototypes and to the reDesign canvas itself. The project was concluded when the 
researcher and entrepreneur mutually agreed on a finalized product prototype and sustainable business model tailored to the design enterprise.

The PAR provided repeated opportunities to test and refine the reDesign canvas, highlighting gaps in the initial canvas design and building blocks. Additions and deletions were made to the building blocks and the features within the blocks as they arose through the design process. The key outcomes of the PAR informed a further iteration of the canvas.

\subsubsection{Interviews}

Interviews with 31 sustainable fashion designers who self-identify as design entrepreneurs and 6 experts within the field of sustainable fashion were conducted to understand how current design entrepreneurs operate. The use of expert opinions in combination with semi-structured interviews is an approach employed by other researchers within fashion sustainability studies (e.g. Winter \& Lasch, 2016). The design entrepreneurs were selected through the use of Instagram and the hashtag \#sustainablefashion. A comprehensive list of sustainable fashion brands was developed, and designers were contacted for participation. Instagram was chosen as it is the most significant social media platform used within the fashion industry and many design entrepreneurs use Instagram as their primary means of exposure. Instagram and the use of hashtags is the most comprehensive approach to discovering sustainable fashion brands. Two criteria were used in the selection of participants: they must be a micro-enterprise, and they must identify as a sustainable fashion brand.

The design entrepreneurs that agreed to participate own and operate small businesses and are leaders in sustainable fashion, principally from New York (12). Others are from London (1), Toronto (7), Montreal (2), Chicago (2), Detroit (1), Berlin (2), Amsterdam (1), Sydney (1), Lisbon (1) and Copenhagen (1). The other 6 experts interviewed included academics (2), independent consultants (2), and product managers (2) from sustainable production facilities. The interviews were semi-structured, 60-90 minutes in length, and focused on how the design entrepreneurs operate and imbed sustainability into their design practice. The interviews also sought to uncover the relationship between sustainable design and their business model, the major barriers, opportunities and advantages of being a sustainable design entrepreneur.

The interviewees were provided with a copy of the second iteration of the reDesign canvas and feedback was obtained. Interviews with experts emphasized their perception and experiences on the role of micro-sized sustainable brands in sustainable fashion and how sustainable fashion 
design entrepreneurs currently work, with a particular focus on their design process and business model development. An interview guide was developed to prepare for and structure the interviews. The interviews were recorded and transcribed. The data collected were coded and analyzed to identify themes, leverage points, challenges, opportunities and key activities. A thematic analysis was conducted, highlighting the similarities and differences between the responses. The key results of the interviews and the role of the feedback received in revising the reDesign canvas are discussed in Section 4. Once the interviews were completed, further iterations of the reDesign canvas were made using the results of the PAR and interviews.

\subsection{Results and Discussion}

This section presents the results. The key results from the development process of the reDesign canvas are reviewed. Using support from the literature and qualitative data gathered in this study, each building block in the final version of the reDesign canvas is described. The key contributions and implications are also addressed.

\subsubsection{The reDesign Canvas}

The reDesign canvas is a design tool that encourages creation and provides support for sustainable fashion innovation specifically targeted to design entrepreneurs. Through the design process, the reDesign canvas promotes innovative business model development and introspection

in parallel with consumer engagement strategies for sustainable behaviour change. The reDesign canvas has been formatted for large scale printing so the design entrepreneurs may use markers and/or attached notes as they interpret, ideate, discuss and implement actions for each of the building blocks. The reDesign canvas is a practical and effective design tool for design entrepreneurs to:

- Apply design thinking and systems thinking throughout their entire business; and

- Identify the opportunities and challenges in sustainable fashion, emphasizing solutions that involve collaboration between the designer and key stakeholders.

The initial draft of the reDesign canvas is presented in Figure 5.3. 


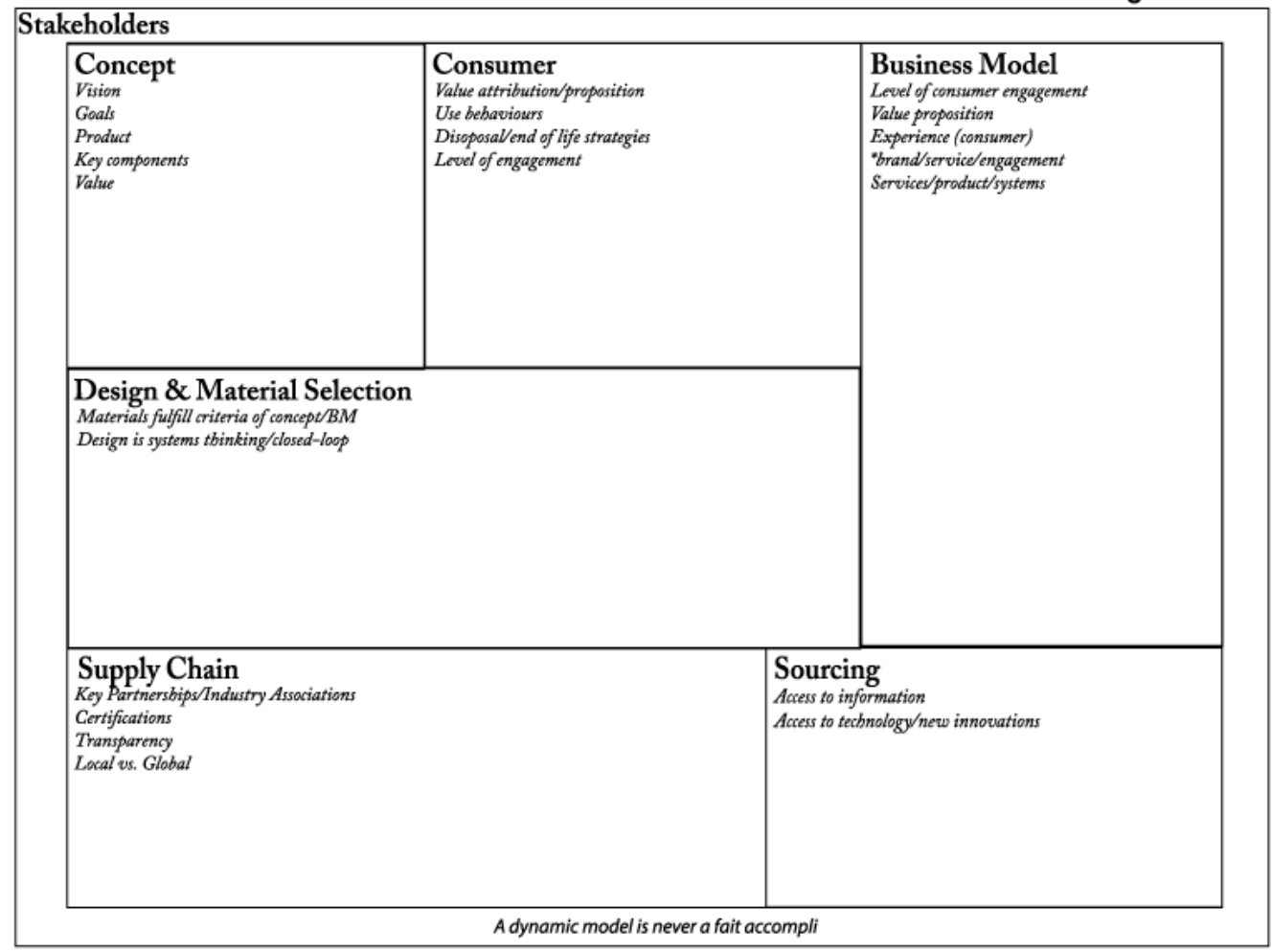

Figure 5.3: Initial draft of the reDesign canvas

The refined version of the reDesign canvas is presented in Figure 5.4. The canvas consists of twelve building blocks: (1) concept; (2) consumer; (3) (de)branding; (4) circular design and economies; (5) business models, value proposition and innovation; 6) revenue streams and costs; (7) sourcing; (8) design and smart material selection; (9) prototypes and product development; (10) supply chain; (11) data management; and (12) stakeholders. Each building block, in turn, consists of a number of features and questions, which are indicated by smaller text under the building block title. Note that several features (e.g., aspired value) are incorporated into multiple building blocks. This underlines the iterative nature of design thinking, where previous assumptions are continually reassessed to ensure their continuing suitability and relevance. 


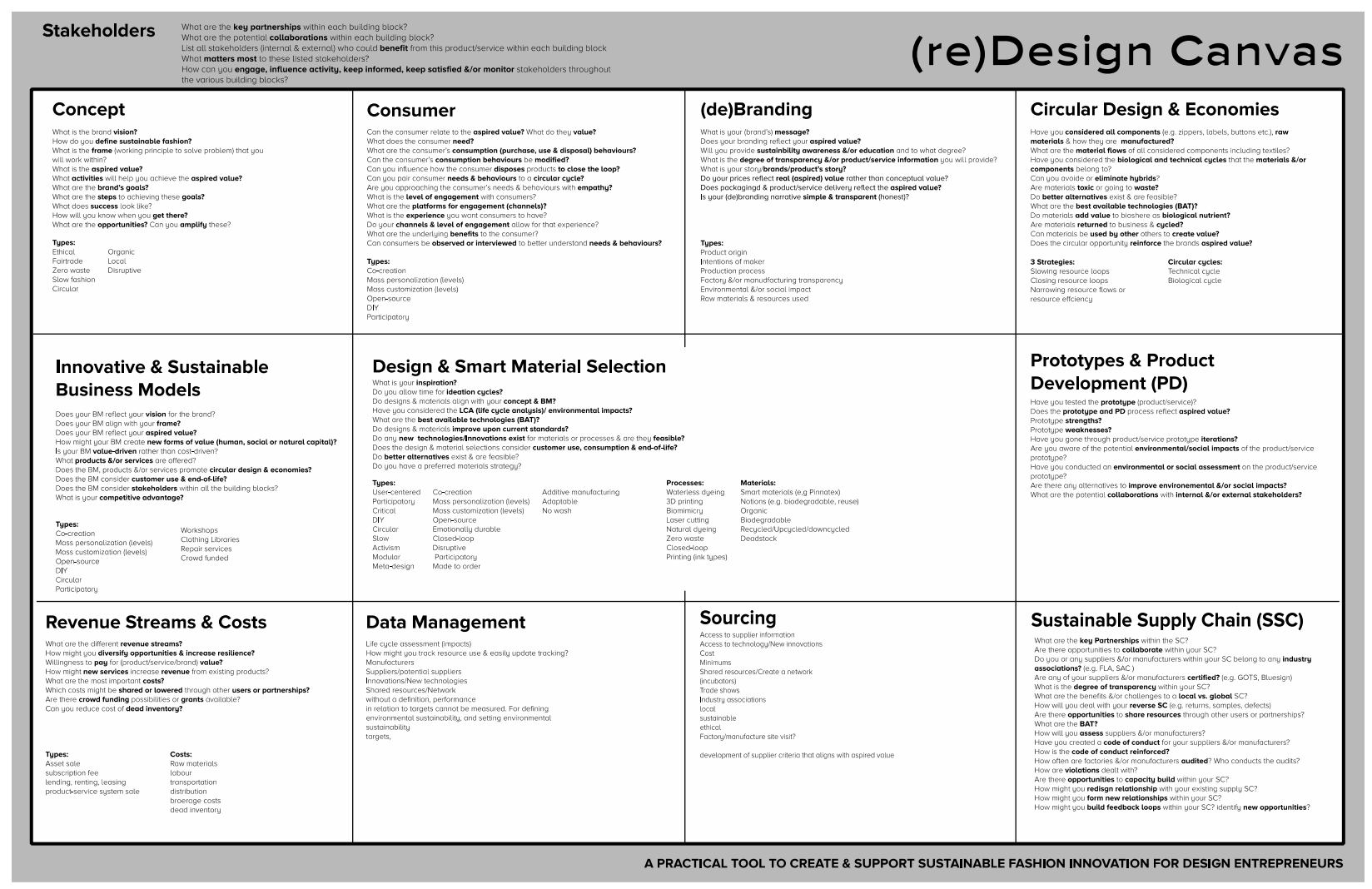

Figure 5.4: Final version of the reDesign canvas

A brief description of each building block is provided in Table 5.1. Detailed

explanations are provided in the following sub-sections. To explain how the building blocks

function, examples from the interviews and PAR are used.

Table 5.1: Description of building blocks comprising the reDesign canvas

\begin{tabular}{ll}
\hline Building Block & Description \\
\hline Concept & $\begin{array}{l}\text { This is where design entrepreneurs develop and outline the broader vision, aspired } \\
\text { value and goals for their brand. This leads the design entrepreneur to better understand } \\
\text { context, the framing of the problem they are looking to address (for sustainable } \\
\text { fashion). }\end{array}$ \\
\hline Consumer & $\begin{array}{l}\text { This building block provides design entrepreneurs the opportunity to explore the } \\
\text { relationship with their consumer. It encourages design entrepreneurs to ask questions } \\
\text { about how to engage, to what level and through which channels so that they may } \\
\text { influence more sustainable consumption and disposal behaviours. }\end{array}$ \\
\hline (de)Branding & $\begin{array}{l}\text { This building block outlines considerations for the design entrepreneur when } \\
\text { developing their branding. The key aspect is ensuring an honest and transparent } \\
\text { account of the fashion brand, products and/or services for the consumer. This block } \\
\text { promotes a shift among brands from investing in marketing to investing in product } \\
\text { development. This allows for pricing to reflect real value rather than conceptual value } \\
\text { created through branding codes, constructed desires and imaginary worlds, thus } \\
\text { illustrating a concept of (de)branding. }\end{array}$ \\
\hline
\end{tabular}




\begin{tabular}{|c|c|}
\hline Building Block & Description \\
\hline $\begin{array}{l}\text { Circular design \& } \\
\text { economies }\end{array}$ & $\begin{array}{l}\text { This building block merges new design strategies with new innovative sustainable } \\
\text { business models. Circular economies and design require that circular products and } \\
\text { services work in tandem with the business model and involves stakeholders like } \\
\text { consumers and producers. This block challenges designers to utilize system and design } \\
\text { thinking in the transition to CE. Designers must consider aspects such as material flow, } \\
\text { best available technologies (BAT), longevity, biological and technical cycles. }\end{array}$ \\
\hline $\begin{array}{l}\text { Business Models, } \\
\text { Value Propositions \& } \\
\text { Innovation }\end{array}$ & $\begin{array}{l}\text { The business model, value proposition and innovation is where design entrepreneurs } \\
\text { can develop the connection between the design, product and consumer in the transition } \\
\text { to a more circular economy. This building block encourages design entrepreneurs to } \\
\text { question how they can deliver new forms of value and innovation to their consumer in } \\
\text { ways that support sustainability. They must align their vision, aspired value, and goals } \\
\text { with the products and/or service and the consumer segment they are designing for. }\end{array}$ \\
\hline $\begin{array}{l}\text { Design and Smart } \\
\text { Material Selection }\end{array}$ & $\begin{array}{l}\text { This building block outlines various design strategies and other considerations for a } \\
\text { sustainable design entrepreneur. These strategies require the same attention as aesthetic } \\
\text { considerations. }\end{array}$ \\
\hline $\begin{array}{l}\text { Prototypes \& Product } \\
\text { Development }\end{array}$ & $\begin{array}{l}\text { Prototypes are an essential characteristic of design thinking and assist the design } \\
\text { entrepreneur in recognizing the strengths and weaknesses of the product and/or service. } \\
\text { This promotes iterations and refinement to ensure the aspired value is reflected before } \\
\text { proceeding to the product development phase. Prototyping also helps to identify the } \\
\text { potential environmental and social impacts of product development and explore } \\
\text { feasible alternatives. }\end{array}$ \\
\hline $\begin{array}{l}\text { Revenue Streams \& } \\
\text { Costs }\end{array}$ & $\begin{array}{l}\text { A design entrepreneur must know their revenue streams, how they might diversify } \\
\text { opportunities and increase resilience within their business. This requires an } \\
\text { understanding of their most important costs and how these costs may be shared or } \\
\text { reduced through other users or partnerships. }\end{array}$ \\
\hline Data Management & $\begin{array}{l}\text { Design entrepreneurs need to consider how and which data are relevant. Sustainable } \\
\text { fashion requires a considerable amount of research and management of this research is } \\
\text { a necessary aspect in building a sustainable fashion brand. It is also important design } \\
\text { entrepreneurs begin to build feedback loops within their supply chain to identify new } \\
\text { opportunities. Management of this feedback data is key for opportunity identification. }\end{array}$ \\
\hline Sourcing & $\begin{array}{l}\text { Sourcing is challenging for any design entrepreneur and this building block outlines } \\
\text { key points for consideration with an emphasis on key partnerships and collaboration. }\end{array}$ \\
\hline Supply Chain & $\begin{array}{l}\text { This block emphasizes stakeholder identification, collaborations, building trusted } \\
\text { relationships and key partnerships. Design entrepreneurs should seek to build a supply } \\
\text { chain with the capacity to build and grow with over time. }\end{array}$ \\
\hline Stakeholders & $\begin{array}{l}\text { Identification of key (internal and external) stakeholders and their (potential) } \\
\text { relationship must be considered for each of the building blocks. This will help design } \\
\text { entrepreneurs in recognizing prospective collaborations and benefits for both the } \\
\text { design entrepreneur and stakeholder. }\end{array}$ \\
\hline
\end{tabular}

\subsubsection{Concept}

With the concept, a designer would begin setting the tone and foundation for the rest of the canvas through the broader vision. The concept work begins with how the designer defines sustainable fashion for their brand.

A key finding from the interviews was how the design entrepreneurs' values and their interpretation of sustainable fashion formed the overall vision for their brand. This was also demonstrated through the PAR: the entrepreneur's idea of sustainability is what drove the 
development of the business model and product design. How the design entrepreneurs defined sustainable fashion was reflected through all aspects of their business, such as material choice, supply chain, sourcing and their business model. For example, one designer defined sustainable fashion as "the best version of how you can do it. For me it's manufacturing as locally as possible and using high quality fabrics and styles that are going to have longevity so that you don't have to go buy" (design entrepreneur 4). Another designer saw sustainable fashion as an opportunity to create awareness with her consumers, stating "you actually have to assume responsibility for your actions as a customer for everything that you buy. And this is something that we want to promote, more responsible customer behaviours" (design entrepreneur 3). Once the designer has established their vision and definition of sustainable fashion, they can begin to work through the remaining questions such as context (framing), aspired value, goals, what success looks like and how they will get there.

Sustainable fashion is an open and complex problem that requires new approaches. Building on Dorst (2011), solutions to these types of problems begin with the end (aspired) value, which is often conceptual in nature. The challenge lays in figuring out "what" (product and/or service) to create and "how" that will be achieved. This forms the basis for the design frame. This, in turn, provides a basis for addressing the remaining questions within the concept building block such as outlining goals, how they will be achieved, determining opportunities, challenges and activities that describe the designer's vision and idea of success. For example, throughout the PAR process, the frame was the key component underlying the entire design of the brand, business and product/services. Often when key decisions were being made, the frame, vision and aspired value were consistently referred to in order to guide decisions and maintain focus.

Using systems and design thinking, the proposed aspired value should be verified against all the building blocks to ensure fit and potential for new forms of value. It is imperative that designers understand how their products, services and brand capture and deliver value within the lens of their adopted construct of sustainability. Moreover, the aspired value should consider and create value for multiple stakeholders simultaneously (Baldassarre, Calabretta, Bocken, \& Jaskiewicz, 2017), including consumers, suppliers, and other key partners. For example, throughout the PAR, the aspired value was to create a women's tote bag using circular design that minimized water use and pollution. The entrepreneur was extremely concerned with water issues and this became the key driving force for the business and design. The entrepreneur 
wanted a business that had positive impacts on communities where water pollution is a major issue due to textile and apparel production.

\subsubsection{Consumer}

While much of the adverse environmental and social impacts of the fashion industry can be attributed to production and distribution, an equal part of the responsibility is borne by consumer consumption behaviours (Roos, Zamani, Sandin, Peters, \& Svanström, 2016). These consumer behaviours are under-researched in the context of sustainable fashion and consumption continues to increase, with the associated high environmental and social costs (Gwozdz, Steensen Nielsen, \& Müller, 2017; Roos et al., 2016). Consuming differently (e.g., purchasing ethically-based products, or products made from recycled materials) is another strategy to reduce environmental and social impacts (Gwozdz et al., 2017).

However, strategies suggested to reduce environmental and social impacts throughout the consumer consumption phase require consumer behaviour change and an understanding of current patterns of fashion consumption (Gwozdz et al., 2017). Many of the questions within this building block are intended for the designer to better understand consumer needs and behaviours so that they may engage with the consumer in new ways to influence behaviour change. This building block invites the designer to imagine how garment design could influence new use behaviours; for example, the use of wrinkle-free or Teflon finishes for clothing reduces the need for ironing or laundering, thereby influencing use and care behaviours. Sustainability researchers argue that focusing on final user needs or the service user wants, rather than solely on the product, can result in lower product impacts (Tukker, 2015). Product end of life strategies, including product reuse, recycling, reprocessing, repair and diverting disposal, are considered as a part of this process and promote systems thinking by linking how the product is designed and its effects through all the building blocks.

The research offered considerable support for this building block. The majority of designers felt that consumer behaviours were extremely important but were unsure about their role in developing more sustainable consumption behaviours aside from awareness. Few designers employed strategies to change behaviours. For example, one of the designers who works with deadstock silks for dresses offers repair services, "all of my dresses come with a lifetime sort of warranty... It's more important to me that they're wearing it, and wearing it often, and if you're wearing it often things are going to happen" (design entrepreneur 18). In any case, consumer 
behaviour change necessitates engagement by the designer. This can occur through online platforms (e.g., brand website, apps, forums, Facebook, or Instagram), through the product design, services and business model such as offering customization or designing for the maker community. Most of the design entrepreneurs interviewed felt that social media platforms are engaging, and an easy way to create awareness and inform their consumer. As one interviewee explained, "[to] show people how [clothing is] made because most people don't understand how things are manufactured... educate people on how things are done, on the process of [manufacturing].... You can do that through blogs and things like that and Instagram" (design entrepreneur 4).

Engagement helps build the relationship between the consumer and brand to impact behaviours that foster a circular economy and closed loop products. This involves diverting resource waste and avoiding disposal by encouraging recycling, repair, reprocessing or upcycling with other industries beyond fashion. This is a challenge for designers as it requires consumers to be more active (Fuad-Luke, 2010). Designers can explore strategies such as user-centred, participatory, do-it-yourself (DIY), co-creation, mass customization, emotional, or critical design. For example, a designer felt that engaging the consumer in the making of the garment created a sense of pride and changed how they valued that garment "whereas in the maker movement [i.e., DIY], people are sharing their ideas; when one person develops something, another person will take it, improve it and share it back. I really like this approach of collectively trying to create something...involving customers is a good way to tell the story" (design entrepreneur 27).

The PAR and interviews also highlight that a sustainable fashion brand requires active participation by the consumer to close the product loop, fulfill a circular economy and develop sustainable consumption behaviours. Thus, designers cannot ignore the consumer experience, needs and consumption behaviours by exploring the implications of this for their fashion brand. For example, one of the design entrepreneurs explores the intersection of consumer engagement and the maker community/DIY (do-it-yourself), "it's that bridge that I want to make - like I've got the skill to do that, I know I can do it and I'm trying to set something up that can help those other people and make them have a more sustainable existence and teach them a skill and build a community" (design entrepreneur 2). Consumer experience is linked to how the designer engages with the consumer. 


\subsection{4 (de)Branding}

Branding is a key consideration in the design process as it is one of the ways in which product offerings may be distinguished from those of other companies. Designers must find that balance between the visual aesthetic fashion feel and an authentic account of the brand's' value and story. Ideally, the authentic account comes from the brands' ability to maintain consistency in their values and vision. Branding within the realm of sustainable fashion requires the product to stand at the forefront where sustainability is articulated through storytelling and the design entrepreneurs' experiences in creating their business and products.

This building block promotes the concept of (de)branding. Designers are encouraged to shift from investing in marketing to investing in product development. This allows for pricing to reflect real value rather than conceptual value created through branding codes, constructed desires and imaginary worlds (De Bruycker, 2016). A reflection of real value promotes an authenticity and transparency by featuring product origin, intentions of the maker, production processes, resources used and environmental and social impact.

As the majority of interviewees noted, sustainable fashion products are often viewed unfavourably in terms of style, quality and price when compared to fashion products, especially fast fashion. While none abandoned sustainability, every designer emphasized that sustainability cannot be at the forefront of branding. Traditional considerations, such as aesthetics and price, cannot be overlooked. As one design entrepreneur explained, a key challenge is in "communicating the story properly and letting people know that we are focused on sustainability without having that be the main focus of our sales pitch. Because we ultimately want to be known as a design company and not a sustainable brand. But at the same time, I struggle with that because I want people to know that we're doing things sustainably in order to encourage them to do it. I'm very torn by that" (design entrepreneur 11). All of the design entrepreneurs agreed and explained that the only way to promote and encourage sustainability is to share information with consumers and with other design entrepreneurs. Storytelling within sustainable fashion is difficult; the dominant approach used by the interviewees was the use of the social media as opposed to their websites to post the daily operations of their brand, communicating transparency and informing their consumers. 


\subsubsection{Circular Design and Economies}

There is a heavy environmental burden associated with excessive consumption of fashion which generates an enormous amount of discarded apparel. Designers must begin to challenge this linear model of use and disposal by adopting approaches for a transition to CE through circular design. It was found that by increasing clothing longevity and direct reuse resulted in the greatest energy and carbon emissions savings (Gwozdz et al., 2017; Roos et al., 2016). A design entrepreneur can adopt several strategies to develop and improve the circularity of their production system and prioritize collaboration with other brands over the supply chain to achieve a more effective circular pattern (Winkler, 2011).

This building block stresses that designers begin to consider aspects such as material flow, best available technologies (BAT), and product longevity. This requires designers to also consider possible business models that can support a transition to more sustainable and circular products and services. This block suggests three main strategies for designers to consider: slowing resource loops, closing resource loops, or narrowing resource flows. Design entrepreneurs are asked to consider the product design and all of its components in order to determine if better alternatives exist and are feasible. This was a key aspect of the PAR where the business model and design were explicitly focused on circular design. The product was designed so that it could be disassembled. "We need to think about will we retrieve the bag and how it can enter another material loop.... We're a brand that takes and gives back to keep a closed looped cycle, no negative impact on the environment" (PAR entrepreneur).

Implementing circular design requires designers to reflect on the resources used and production processes. Alongside the design strategies and considerations, designers need to think of new business models that would accommodate the sustainability of the designs and whether the design and business model reflect their aspired value. Design entrepreneur 27 had an innovative business model that made use of new technologies, maker spaces and self-assembly, "by offering people a chance to influence and be part of the design and production process of their garments will create much more attractive products then the current mass-manufacturing industry does.... [Our] garments are designed on the spot by our software whenever someone wants to buy one. This way they are produced only when they're sold." 


\subsubsection{Business Models, Value Proposition and Innovation}

The business model, value proposition and innovation is where design entrepreneurs can develop the connection between design, the product and/or service and consumer engagement in the transition to a more circular economy. The development within the concept building block such as value proposition, aspired value, frame, vision and goals will inform the generation and/or introspection of a business model. This building block encourages designers to question how they can deliver new forms of value and innovation to their consumer in ways that support sustainability and circularity. The deeper integration of environmental and social needs within business activities and innovative practices is necessary for a fundamental shift towards sustainability (Frank Boons \& Lüdeke-Freund, 2013). A business model that is user-centred allows for solutions that are meaningful for consumers and is profitable for businesses (Baldassarre et al., 2017) while moving away from the traditional model that focuses on increasing production volume to fuel growth and consumption. Meaningful solutions are supported "by involving potential consumers, user and/or other stakeholders in an experimental and iterative design process" (Baldassarre et al., 2017, pg. 175). Business models must adapt and be flexible to encourage innovation and collaboration among other sustainable fashion SMEs and consumers.

The literature, the PAR, and the interviews all indicate the importance of placing consumer relationships at the centre of the business model. All of the designers interviewed except for one still functioned with the typical linear business model. The consensus was familiarity, as that was what they had learned in school and through internships.

There are other models. Design entrepreneur 27, who was particularly concerned with overstock and post-consumer waste, developed a business model and value proposition structured around the principles of waste minimization, product-service systems (PSS) and usercentred design. An innovative cutting method for assembly drives the business model and value propositions. Designs can be bought made-to-order or as digital design files that can be downloaded. The consumer then uses the file to have fabric laser cut into the specific pattern pieces which are designed for easy no-sew assembly by the consumer. PSS provides added value to the product life cycle and provides enabling platforms for the consumers while re-orientating current unsustainable trends in production and consumption (Manzini \& Vezzoli, 2002, 2003). This alters garment value by involving the consumer in the actual creation of the garment. Emotional attachment is created through engagement and participation, potentially increasing 
garment longevity and reducing the need for new acquisitions (Chapman, 2005, 2009; Fletcher et al., 2012; Karell, 2013).

Focusing on the business model and value proposition can lead to more opportunities, meaningful solutions and the potential to move beyond incremental innovations while offering sustainable products and services. This building block serves to strengthen a designer's ability to pursue sustainable fashion by integrating their construct of sustainability, aspired value, vision and goals into a business model and value proposition.

\subsubsection{Revenue Streams and Costs}

The revenue streams and costs are the foundation of the monies a company generates through consumer sales and the associated costs. There are many hidden costs in design (e.g., prototypes, sampling, grading) and a need for capital up front for activities such as material purchase for prototypes, sampling, pattern development through to brokerage fees, and deposits for production. Sustainable fashion design entrepreneurs face additional barriers as sustainable textiles and fair living wages increase production costs. As one entrepreneur explained, "[our] decision to pay ethically for the production of [our] clothes while letting [our] profit margins decrease has been a challenge... as a fashion startup. Mostly business people... they're kind of shocked or ask, well why would you do that when you can make something cheaper and make more money?" (design entrepreneur 29).

About half of the design entrepreneurs specifically expressed the challenges in fighting the dominant mindset of cheap production, low costs and inexpensive products. As one interviewee noted,

people today expect to be able to buy clothes so cheaply... people just expect to be able to buy a sweater that cost $\$ 15$ from Joe Fresh...It's not that cheap to buy these things because it costs money to pay people properly and to do things in a way that is fair... Cutting out those middlemen was a way that we were able to cut costs because we didn't want to be charging $\$ 100$ for a tee shirt. We wanted it to be affordable but at the same time still some people think \$30-35 for a tee shirt is expensive, but really that's like to us how much it should cost based on what we have to pay people (design entrepreneur 7).

Design entrepreneurs face many barriers in adopting, accessing or financing many of the sustainable strategies, processes or products for their business. Small sized brands often 
struggle. Many of the designers (e.g., design entrepreneurs 2, 3, 8, 12, 23, $27 \&$ 28), expressed frustration in regard to awareness, access and capital to take advantage of new sustainable innovations as the majority of production within the industry is tailored for larger enterprises.

The sentiment of the industry not being conducive to startups was felt by all the design entrepreneurs interviewed in this study. Overall, the design entrepreneurs' sentiment was that sustainable fashion design entrepreneurs face additional challenges in terms of cost and pricing strategies. This did not deter design entrepreneurs but reinforced the ability to prioritize and plan accordingly to achieve their aspired values and to operate their business accordingly.

\subsubsection{Sourcing}

The majority of the designers stressed that sourcing is where they had the most difficulties, especially in locating and acquiring information regarding sustainable suppliers. As the interviewees explained, many sustainable suppliers are smaller in scale and do not have an online or highly visible presence. This makes it extremely challenging for small sustainable fashion brands to source the resources needed.

This building block emphasizes that designers must identify internal and external stakeholders with whom they could potentially partner, creating a network of shared information and resources. Creating a network to share resources increases access and information about suppliers, technologies and innovations. Designers are also encouraged to seek out and participate in initiatives such as trade shows, design incubators and industry associations. Building a network of small sustainable fashion design entrepreneurs allows for the potential to bundle sourcing activities. Bundling orders increases access to certain resources that may not have been accessible to a single designer due to minimums. This allows designers to meet minimum orders and potentially reduce costs. One of the design entrepreneurs who manages a design incubator spoke to the benefits of creating a collaborative sharing community and its impact on sourcing:

We [share orders] here. I share with [design entrepreneur 12] - at least we're trying to share some fabrics and orders. I think [design entrepreneur 12] and [design entrepreneur 13] do it too. You get a better price, you get better shipping, you get your order a little bit faster and it just makes it a lot easier for the mills as well. And when there's no overlap... like [design entrepreneur 12] is a men's brand and I do womenswear so there's no conflict there. We also design very differently, so what I 
produce with the same fabric is going to be very different from what he's producing.

I think there's less threat whereas other designers just feel a threat that isn't there. (design entrepreneur 11)

There was a difference between those who belonged to an incubator ( 5 designers and the PAR entrepreneur) and those who did not. Designers within an incubator setting did not report the same sourcing issues as others. Information regarding suppliers was readily available, more experienced designers helped with questions regarding supplier evaluations, material types and qualities, and encouraged bundled orders.

The PAR and interviews also emphasized the importance of a sustainable fashion community. Designers sourced materials and manufacturers predominately through the sustainable fashion community. The questions in this block can help designers to determine criteria and evaluation methods to vet new suppliers. The aspired value of the designer and brand will aid in determining which criteria should be used, what questions to ask, when to use and develop a code of conduct and what evaluation method could be applied.

None of the designers interviewed used code of conducts. However, most of the designers reported a very close relationship with their suppliers, were aware of any certifications they had, and were confident in their labor and production practices. The rest of the designers made their products in-house. Designers need to be informed regarding issues such as certifications, environmental and social regulations, living wage initiatives, and auditing procedures in their supplier relations. This is an ongoing process.

\subsubsection{Design and Smart Material Selection}

The emphasis on design is the foundation of the reDesign canvas. The sustainability of a product depends mainly on the choices made in the early phases of design or product planning (Ramani et al., 2010). It is important to note that by working through the rest of the reDesign canvas, much of the groundwork for the design and material selection would already have been completed.

Choosing materials that have already been screened can be very beneficial. For example, material can be sourced from a small-scale producer who is certified organic and ethical for their wool products. The design entrepreneur would only need to ensure sustainable production of the yarn into a garment and can focus on closing the loop using strategies such as designing for biodegradability or disassembly once the product is no longer in use. This can help link the 
design and materials selection process to the business model, (de)branding and consumer engagement.

What was evident from the interviews was the design entrepreneurs had vastly different approaches to material selection. For example, design entrepreneur 14 explained how material selection arose from supplier approaches and environmental impacts

Part of what I want to do is to revisit all [supplier] facilities, so I know exactly what they do, and how things are made, and what sort of machineries and how much water they're using all these things, and I can meet the workers and stuff.... I think what helps is that we actually go to these places and see how things are made. I mean that way, you know exactly what you're dealing with, and that will help you decide, change your material choice... because of the way it's made.

\subsubsection{Prototypes and Product Development}

Prototypes are an essential characteristic of design thinking and assist the design entrepreneur in recognizing the strengths and weaknesses of the product and/or service. Iterations are performed to address strengths and weakness, to refine aspects of design, and to ensure the aspired value is reflected before proceeding to the product development phase. As a designer moves into the product development phase, interfunctional collaboration is a critical success factor in the adoption of sustainable innovation (De Medeiros, Ribeiro, \& Cortimiglia, 2014). This includes the integration of various stakeholders (both internal and external) in the product development process (De Medeiros et al., 2014).

This block focuses on engaging designers as to the adverse impacts material, production processes, use and disposal have and how they may mitigate them once identified. To help address the many challenges that arise during product development, many designers use guidelines, checklists and analytical tools. However, the use and application of eco-design tools, methods and techniques by small sized firms is limited (Baumann et al., 2011; Knight \& Jenkins, 2009). Almost none of the interviewed design entrepreneurs used tools, such as Ted's Ten (Textile Environment Design, 2006), the Nike Maker App (Centre for Sustainable Fashion, 2013), or the Sustainable Apparel Coalition's Higg Index (Sustainable Apparel Coalition, 2017), and the majority were not aware of these tools. The design entrepreneurs had used environmental impact data for aspects such as material choice which was retrieved primarily by sustainable fashion websites or books. One of the five designers who was aware and did use tools such as the 
Higg Index or Nike Maker App noted, "when it comes to the Higg Index I was reading about it but it's a very complicated process... Maybe the Making App from Nike, for me it's better because it's a bit more visual and the information is more easily displayed" (designer entrepreneur 3). This is a key aspect that design entrepreneurs need to consider if they are to reduce environmental and social impacts from production to disposal.

Prototyping or sampling is a key factor in developing well-made quality garments, an important characteristic of sustainable fashion apparel agreed upon by many of the design entrepreneurs. Design entrepreneur 7 summarized this process with their manufacturer:

we demand a lot from him because some of his other clients are big supermarkets in France that are producing big quantities and don't really care about quality control that much. [We are] literally on the phone with him every other day like "we've got a tee shirt and the hem is lopsided," and we're really on top of it. He sometimes must want to kill us. But most of the time he's actually really helpful and really invested in what we're doing because we're trying to help the industry and because we care about quality and we really care about what they do. And when we do turn around and say this is amazing then it means a lot more too... we're just trying to work towards better processes and improve a little bit with every production run.

\subsubsection{Supply Chain}

The supply chain is one of the most crucial aspects in forming sustainable fashion brands and is heavily informed by the other building blocks within the canvas. This block emphasizes collaborations, building trusted relationships, consideration of key stakeholders (internal and external) and key partnerships. In selecting suppliers, research shows there is no difference between small and large firms in the perceived importance of environmental criteria (Min \& Galle, 2001). However, SMEs do face specific challenges in building a sustainable supply chain and in sustainable supply chain management.

The feedback from the interviews conducted in this study showed that design entrepreneurs struggled with a lack of financing, most suppliers and manufacturers are geared towards large firms with high production volumes, difficulties in exploiting technology, ability and capacity to gather information, small production runs, and constrained managerial capabilities. For example, 
suppliers have high minimum orders for fabrics, pressing design entrepreneurs to purchase in much larger quantities than what is needed. As one design entrepreneur explained, "That's still one of our biggest issues. Even as our quantities are growing these factories won't even speak to you unless you're doing multiple tens of thousands or in the hundreds of thousands. We're getting there with a few things but when you're doing a brand new product you don't want to do 20,000 of it" (design entrepreneur 7). This challenge was echoed by most of the designers; for example one noted, "we're definitely limited because of the quantity, it's definitely a struggle to put together a supply chain when your minimums are so low" (design entrepreneur 10).

Today, as consumers are more aware of environmental and social impacts, the sustainable behavior of a company's suppliers is considered by its stakeholders (Pitchipoo, Venkumar, \& Rajakarunakaran, 2015; Seuring \& Müller, 2008). Typically, information regarding a brand's supply chain, sourcing and design practices is highly guarded. The interviews emphasized this is not the case for sustainable fashion designers. As one design entrepreneur explained, "The whole idea behind that is to educate my consumers about where it's coming from and hopefully encourage them to ask questions but also to allow other designers to come and tap into my supply chain and use it for themselves because I'm not going to support it myself. So my vendors appreciate that a lot" (design entrepreneur 11).

Supplier evaluation is a key aspect of sustainable supply chain management (Winter \& Lasch, 2016). The first step in evaluating a supplier is prequalification in which appropriate suppliers have to be identified (Pitchipoo et al., 2015). It was found that each of the design entrepreneurs, including in the PAR, visited key suppliers (garment manufacturers) prior to establishing a working relationship. As expressed by design entrepreneur 30, "going to manufacturers locations overseas is part of accountable supply chain management, which is difficult for large fashion companies to achieve, let alone small startups." Another explained, "I visit my factory in Turkey at least 2 or 3 times a year and I always talk with their workers. The conditions are amazing and when I design for other people... I have to travel and I see completely different conditions which is horrifying for me".

Collaborative efforts can also influence the use and implementation of certifications. Many certificates exist for both designers and suppliers (e.g., Global Organic Textile Standards, Bluesign, or C2C) (Bluesign, 2016; Cradle to Cradle Products Innovation Institute, 2018; GOTS, 2016). Certifications may become even more important as requirements for product traceability grow (Lakhal, Sidibé, \& H'Mida, 2008). Designers may also aid suppliers achieve certification 
standards by working with closely to optimize a certain material or process. Some of the knitwear designers interviewed in this study (e.g., design entrepreneurs 5, 7, 10, 13, 14 \& 25) worked with small scale farmers, spinners and/or weavers in rural communities.

Designers may want to consider pooling resources and sharing the costs of evaluations, training, capacity building, certifications, diffusion of technology and new possibilities for efficiency gains and innovations. SMEs can often be more flexible and responsive than larger firms. Memberships to design incubators and accelerators can support a grouped local system of production. Among the design entrepreneurs interviewed, those who belonged to an incubator or partook in accelerator programs demonstrated potential capability as sharing of resources and pooling for costs savings was already taking place.

\subsubsection{Data Management}

This building block encourages designers to begin collecting data regarding the environmental and social impacts of materials and resources used. This can aid the designer in selecting materials and resources that align with their interpretation of sustainable fashion, goals and aspired vision.

Data management was not a common practice among any of the designers interviewed. However, this was key to the development of the supply chain throughout the PAR. Some of the designers interviewed in the study (e.g., designer entrepreneurs 3 \& 30) used environmental and social impact data for material or process selection. For example, one designer used this type of data to inform what yarn type would be used for knitwear products (design entrepreneur 13). Another designer (design entrepreneur 18) in an effort to reduce waste and minimize environmental and social impacts of textiles only used remnant end-of-bolt and deadstock fabrics from a local supplier. This influenced her design practice and business model as many garments were one of a kind with small runs and could not be re-ordered or replicated.

In the interviews, many of the design entrepreneurs cited an interest and understood the benefits of developing data management system for continual improvement but lacked the skills, time and finances (e.g., design entrepreneurs 3, 7, 23, 30). A goal of designers should be to upgrade their management skills, and their capacity to gather information, coordinate data from suppliers (collaborative efforts would work best here) and their technology base. 


\subsubsection{Stakeholders}

Stakeholders are a key consideration throughout the reDesign canvas. In the pursuit of a sustainable fashion brand, it is necessary for design entrepreneurs to identify and consider key stakeholders within each of the building blocks. Collaboration, sharing resources and capacity building with stakeholders can accelerate progress and reduce costs in developing sustainability (Bos-Brouwers, 2010). Scalability and economies of scale are the driving forces of how the industry is structured and operates. For new sustainable technologies and innovations to become implemented at the scale necessary to inflect positive change, collaboration is essential.

Cooperation and collaboration with stakeholders are key to the development of sustainable products, services, supply chains and business models. Identification of the stakeholders provides design entrepreneurs an opportunity to reflect upon the (potential) relationship with them and their brand; each relationship will differ depending on the stakeholder (Roos et al., 2016). For example, design entrepreneur 10 leans on her community, those who share the same values, to learn about new sustainable developments: "if you have any questions or struggles or just want to bounce ideas off, you have somebody to call". Most of the design entrepreneurs exhibited a willingness to share resources as a means of supporting the sustainable design community, "even if it's just support... or wherever we can help like a contact here or there we'll do it" (design entrepreneur 7).

Designers who worked with natural fibres highlighted the benefits of capacity building with key stakeholders in their supply chains. For design entrepreneur 25, this means supporting producers in scaling: "the hope too is that everyone gets to just focus on doing what they're good at, and then collaborating... the weaver that I work with, she's very on board to amp it up to a full-scale production. So, we're going to help her do that." Collaboration with other designers can help with processes such as certification. As described by design entrepreneur 7, "working to get organic certification in Egypt is something that we would really like to do... so if we can band together with [other] partners and try to make this happen, that would be really cool." The majority of the design entrepreneurs saw value in sharing resources, collaborating and capacity building with stakeholders for immediate and future benefits.

\subsubsection{Proposition Verification}

The propositions introduced in section 2 provided parameters and guidance in the development of the design tool, the reDesign Canvas. Throughout each iteration, the propositions 
continuously and systematically were verified against the building blocks that make up the canvas. All of the propositions with the exception of proposition 3, are easily verified by ensuring the features and questions within the building blocks support each proposition. Proposition 3 ensures the design tool functions with certain parameters, reflecting a holistic perspective and systems thinking. Throughout the design tool development process, the linkages and interactions of the building blocks and their position relative to the whole (i.e., the canvas) was a significant consideration. The canvas was chosen due to the widespread adoption of canvas-type tools for group brainstorming activities, design thinking and development. As a visual tool that invites engagement, a canvas can support the documentation of a system and its fundamental components. Feedback from research activities was incorporated in the development and organization of the building blocks into the reDesign canvas, verifying proposition 3. Verification of this proposition will be an ongoing process as feedback received from use will be used to continually refine the form and function of the canvas. For example, a sustainable fashion design tool could be created in various forms such as an interactive website, an app, a canvas or set of cards

\subsection{Conclusion, Limitations and Future Research}

This paper develops an original design tool, the reDesign canvas, to support design entrepreneurs in developing sustainable fashion enterprises. The canvas provides a clear, concise mechanism for structuring thinking and discussion about the opportunities and challenges design entrepreneurs face in this context. The canvas was developed based on an indepth review of the academic literature and the collection of new empirical data. The data were gathered through both participatory action research (PAR) and interviews with experts in sustainable fashion. Both the PAR and the interviews were used to test and refine the reDesign canvas in order to ensure it meets the needs of sustainable design entrepreneurs operating microsized companies. The final version of the canvas is based on 12 building blocks that a design entrepreneur would encounter in building a sustainable fashion brand.

The paper makes a number of contributions to the academic and practitioner knowledge bases. From an academic perspective, the reDesign canvas fills an identified gap in the literature on the need for design-focused tools targeted to entrepreneurs. The development of the canvas brought together a great range of literature, including sustainable business models, SMEs, circular economies, sustainable supply chains, and sustainable fashion, among others. The systems and design thinking perspectives provided the theoretical foundation needed to make 
sense of this deeply fragmented literature. Given the rapidly evolving field of sustainable fashion, the qualitative data collected as a part of this study were needed to contribute new insights into the challenges facing small sustainable fashion brands, and how they may be addressed in a business based on personal ethics, morals and values. The reDesign canvas thus provides a needed bridge between academic and practitioner work. From a practitioner perspective, the reDesign canvas provides designers with a strategic and entrepreneurial tool that is visual and caters to how designers work and the design process. Designers have the freedom to work through the building blocks in an iterative manner that suits them and their business while challenging creative application to aspects beyond product design. Over time, the application of the reDesign canvas may help design entrepreneurs build sustainable fashion brands. The reDesign canvas presented is a design tool, which has been created, described and offered as a possible means to overcome the barriers that fashion sustainable fashion design entrepreneurs face.

Overall, the paper contributes new knowledge that can prompt rethinking of previous assumptions as to the role of SMEs in developing a more sustainable fashion industry. Many of the existing tools for design in a sustainable fashion context are either too complex, overly conceptual, require experts to apply, have a high cost, were created for large corporations or fall short in holistically supporting sustainable fashion design entrepreneurial practice, such as emphasizing stakeholder involvement, consumer engagement and a transition towards a circular economy. This paper addresses this gap by proposing and exemplifying a holistic approach that harnesses the creative powers of designers for solutions to sustainability at the SME level. The reDesign canvas emphasizes system and design thinking by integrating sustainable business model innovation practices with fashion design practices. This provides a necessary and relevant baseline for future research into how the fashion industry can provide the necessary support for small sustainable fashion brands and design entrepreneurs.

This study is, of course, not without its limitations. The reDesign canvas would benefit from more use and feedback, as further iterations could improve upon any missing features or shed light on those that may be less relevant. This might be written about any new model, but the point is particularly salient given the ongoing rapid evolution of design practices in sustainable fashion. Future work can also focus on adding depth to the building blocks contained in the current version of the reDesign canvas. For example, future work could focus on developing a specific set key performance indicator that are relevant and manageable for a sustainable design 
entrepreneur. Key performance indicators offer the opportunity to investigate the notion of what constitutes a sustainable fashion brand. While many fashion brands have begun to introduce small sustainable collections, such as offering a selection of organic t-shirts, research has yet to explore what a sustainable fashion brand actually entails and how progress towards to that could be measured. Similar elaboration could be undertaken for other building blocks in the canvas. 


\title{
6. CONCLUSION
}

\begin{abstract}
This chapter summarizes the major findings of this research. A review of the four objectives and the resulting manuscripts are provided. There is a discussion of the results in relation to the overall research question of this study. This chapter includes a series of reflections and recommendations for future research regarding sustainable fashion MSE designers and their role in the development of a responsible fashion system.
\end{abstract}

The purpose of this research was to explore the potential of sustainable fashion MSEs, and the design entrepreneurs who own and operate them, in creating a responsible fashion system. This was a study of their design practices and existing sustainable fashion design strategies and tools. This exploration into sustainable fashion design practices of MSEs was guided by four objectives. The objectives were met through the development of four studies, resulting in four manuscripts: 1) Theorizing the Fashion System; 2) Tools for Sustainable Fashion Design: An Analysis of their Fitness for Purpose; 3) A Case Study of Sustainable Fashion MSEs and their Sustainable Design Practices; and 4) The reDesign Canvas: Fashion Design as a Toolfor Sustainability. In this chapter, the conclusions regarding each research objective are summarized first. This is followed by a reflection of the results, recommendations, limitations and future research.

\subsection{Theorizing the Fashion System}

Fashion studies is a relatively new area in comparison to the more traditional and established disciplines within Academia. As a largely practice-based discipline, theory that uses a broad multi-disciplinary perspective is limited. Fashion often sits at the nexus of differing social, psychological, economic, and cultural contexts and has been studied through a variety of lenses. The first objective was established to provide context for the creation of a sustainable design framework through this theoretical review and development of a fashion system theory. This work sought to conflate the varied lenses and theories into a single conceptualization, forming a framework with design as the nexus. The single conceptualization seeks to represent and outline how the fashion system currently is constructed. Fashion is viewed as multi-dimensional and as 
such, five dimensions were identified to guide the approach. It is argued that four of the dimensions contain pairs of diametric counterpoints, that provide the fundamental theoretical underpinnings, while design forms the interconnection. The four distinct dimensions and counterpoints are: (1) Agency: Creator - Consumer; (2) Supply Chain: Production Consumption; (3) Merchandising: Brands - Cues; (4) Motivation: Art - Commerce. These form the basis for a unified theory of the fashion system.

The fashion system conceptual model facilitates a holistic perspective in identifying possible relationships and connections through the use of a model for the development of a responsible fashion system. This model is rooted in systems thinking to provide an understanding of how sustainable fashion design practices can affect consumption, business practices and design tool development. The model can be applied to the diversity of areas of research interest in the discipline and fulfills the need for translation among the theoretical foundations which have been applied in Fashion.

\subsection{Tools for Sustainable Fashion Design: An Analysis of their Fitness for Purpose}

The second objective of this $\mathrm{PhD}$ research was to analyze existing tools for sustainable fashion design. Understanding the complexity of sustainable fashion issues can be overwhelming and a barrier for fashion designers. A number of tools for sustainable fashion design have been developed to aid designers in the integration of sustainability into their design practices. An analysis of their fitness for purpose was conducted to understand their strengths, suitability, applicability and opportunities for improvement. To undertake the analysis, an innovation framework and a five-dimensional model of sustainability specific to fashion were created. Four levels of innovation have been proposed: 1) product-level, 2) product service social systems level, 3) systems level, and 4) resilient, adaptable and circular level. We propose a novel sustainability framework specific for fashion that includes five dimensions: 1) environmental, 2) economic, 3) social, 4) aesthetic, and 5) cultural.

This study contributes the first comparative analysis of existing tools for sustainable fashion design, resulting in the identification of three categories (archetypes) of tools: Universal, Participatory, and Assessment. Using the archetype categorization may facilitate designers in identifying the most appropriate type of tool for a specific circumstance, depending on context and need. There also advantages for developers in using the archetype categorization to create tools that work with other tools. Using the proposed frameworks, it was found that the majority 
of the Strategies presented by the Tools were limited to product-level innovations. The impact of Strategies categorized at the product-level is limited to improvements rather than transformational change. It is thus suggested that designers strive to - and developers aim to include - a variety of sustainability strategies from the various levels of innovation to transform fashion into a responsible system.

\subsection{A Case Study of Sustainable Fashion MSEs and their Sustainable Design Practices}

The third objective of this research study was to explore how sustainable fashion MSEs currently work. The aim was to examine designer entrepreneurs' knowledge and how sustainability was incorporated into their design practice, strategies or tools used, and to understand how their choices impacted business operations. This study also sought to identify any barriers the design entrepreneurs encounter in operating a sustainable fashion brand. Design entrepreneurs who own and operate a sustainable fashion MSE were interviewed using a semistructured format. Using a qualitative content analysis, themes and patterns into the types of sustainability strategies used and challenges were identified.

Results indicate that the design entrepreneurs in this study are highly committed to sustainability and operate their MSEs and design according to their values and beliefs as they relate to how they define sustainable fashion. The designer entrepreneurs in this study possessed a variety of positive characteristics such as flexibility, independence, innovative, creative, resourceful, highly collaborative and optimistic. They are driven to provide the best alternative that they can to traditional fashion options available on the market. The designers' favored local production, transparent processes, the use of ethical labour and alternative business practices such as working with an anti-fashion calendar model. Challenges encountered by the designers include issues around cost, pricing, financing and access to current sustainable technologies, innovation and information. The current structure of the fashion industry favours large companies. This study has revealed that sustainable MSEs are a positive contribution to the sustainable fashion space but need support with the resources, systems and structures to succeed in their endeavors for a responsible fashion system. This includes supporting local production network activities and fostering collaboration with all stakeholders. 


\subsection{The reDesign Canvas: Fashion Design as a Tool for Sustainability}

The final objective was achieved through the creation of a sustainable fashion design tool, The reDesign Canvas. It was found that many of the existing tools for sustainable fashion design are too complex, overly conceptual, require experts to apply, have a high cost, were created for large corporations, or fall short in holistically supporting sustainable fashion design entrepreneurial practices. Informed by design thinking and systems thinking, the Canvas was developed based on an in-depth review of the academic literature and the collection of qualitative data. Qualitative data were gathered through both participatory action research (PAR) with an entrepreneur and interviews with design entrepreneurs. The tool was developed through a real-world application in designing a fashion product (PAR) and interviews with the design entrepreneurs. Both the PAR and the interviews were used to test and refine the reDesign canvas in order to ensure it meets the needs of sustainable design entrepreneurs operating micro-sized companies. The final version of the canvas is based on twelve building blocks that a design entrepreneur would encounter in building a sustainable fashion brand. The reDesign canvas aims to support design entrepreneurs in developing sustainable fashion enterprises and can help advance both the theory and practice of sustainable fashion design.

\subsection{Reflection}

Throughout the development of the industry, industry efforts have sought to diminish costs and complexity by dividing up the tasks it takes to make a garment or a shoe. Each individual has their own discrete component of a unit that they repetitively perform. Units are mass manufactured in a piecemeal manner with economies of scale. Moving production overseas, dividing work into further disparate tasks has only served to spread complexity, making it harder to see and far more inflexible. In recognition of this complexity, a change is needed.

Sustainable fashion MSEs, as this research has shown, are flexible, innovative and operate outside this complex system in favor of simplicity, transparency and balance. Their desire to build businesses that operate outside of the current industry structure and practices stems from their frustrations. All the design entrepreneurs started their business because of their frustration with the industry status quo and/or their inabilities to effect sustainability change with previous employers. 
They are highly committed to developing a responsible fashion system but largely lack the resources to do so in a meaningful way as they are antithetical to the current system. The fashion industry is slanted for the financial success of large global fashion firms, as demonstrated by the challenges the sustainable fashion MSEs in this study face. As the industry is increasingly under scrutiny for its contribution to the steep decline in the overall health of our planet, tools have been developed to mitigate this. These tools are intended to aid the transition to a sustainable fashion industry but are also primarily developed for the use by these multinational firms. MSEs are largely unaware of many of these tools and for those who are aware, cite lack of time and resources to use and implement the tools. There is still a disconnect between sustainability, fashion and fashion design, as supported by current research citing slow progress. We are simply not transitioning at a fast enough pace (Global Fashion Agenda, 2019).

A key distinction between sustainable fashion MSEs and large global firms is where their efforts are concentrated regarding fundamental practices for sustainability as depicted in Figure 6.1. Fundamental sustainable fashion practices are demonstrated through four primary modes: 1) creative design, 2) social, 3) environmental and 4) business. Larger firms focus on environmental initiatives and reducing impacts using life cycle assessments, performance indicators, targets and goals throughout their supply chain (Kozlowski, Searcy, \& Bardecki, 2015). The negative social impacts are also addressed, but not significantly, and have yet to be successfully eradicated, as slave labour issues, for example, continue to persist in global supply chains (Minney, 2017). This research demonstrates sustainable fashion MSEs as focusing on creative design, business, and social aspects primarily. They focus on creative design approaches and new business models for innovative solutions to sustainability. They are cultivating cultures of sustainability by engaging with their local communities and consumers to support local production networks, create awareness around fashion sustainability and encourage sustainable behaviours. 


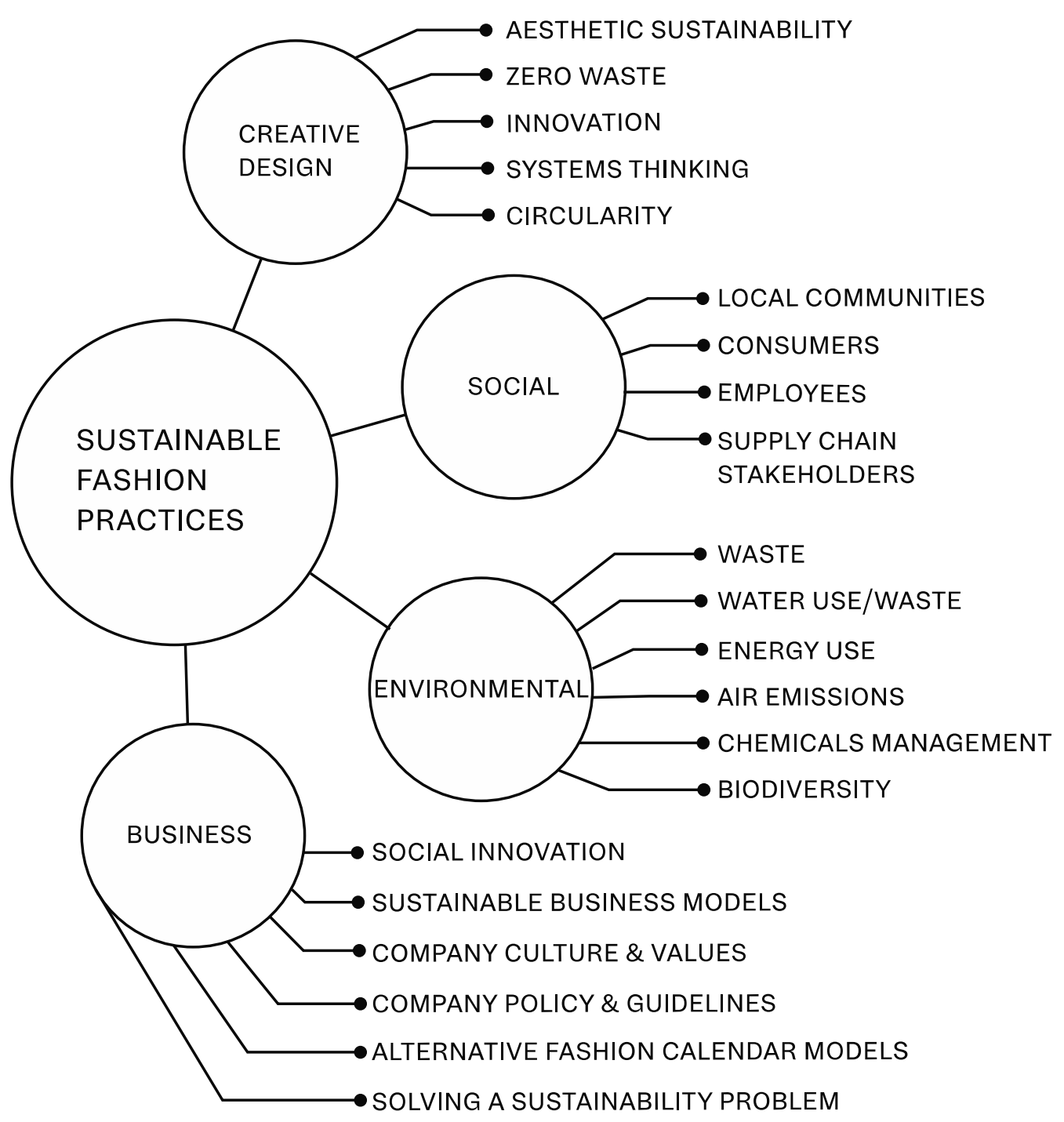

Figure 6.1: Description of key sustainable practices

The comparative analysis of the tools for sustainable fashion design support these conclusions. For example, the tools belonging to the Quantifiable archetype focus on impact assessments and are built for larger brands with complex global supply chains - and these are the types of fashion businesses that typically use these types of tools. A prime example is the Higg Index by the Sustainable Apparel Coalition where Nike's Material Sustainability Index forms the foundation. The contributor's and users of the Higg Index, as shown on their website, is foremost large recognizable global firms (Sustainable Apparel Coalition, 2019). Sustainable fashion MSEs do not have the time or resources to invest into impact assessment and reduction strategies. The focus of tools such as Ted's Ten (Universal archetype) are qualitative and encourage creative 
design, implementing sustainable business models, social innovation, and community engagement. These types of tools are easily used by MSEs as they are developed with designers and MSE case studies. These tools do not require designers to be experts and perform assessments on impacts or use of LCA's for example. Instead, these tools focus on small, manageable strategies such as creating awareness with consumers, living wages, reducing waste through garment design and better sustainable material solutions. The sustainable strategies that make up the various tools are indicative of what type of fashion business would likely use them.

Sustainable fashion MSEs have the flexibility and simplicity to make the necessary adjustments to operate sustainably. In establishing cultures of sustainability, they do not seek to grow for growth's sake into large multinational firms. A significant amount of the design entrepreneurs were previously employed, working for established fashion businesses. Their dissatisfaction with how the industry typically operates is what inspired them to begin their sustainably focused fashion businesses. They are focused on solving the problems of "unsustainability" in the industry. Their approach, beliefs and values toward business ethics, the craft of creating garments and ideas of what fashion should be differ from those who dominate the industry. For example, sustainable fashion MSEs who seek to reduce waste do so through design means while a larger company is more likely to reduce waste through production methods rather than design. This is extremely valuable and should be nurtured. MSEs demonstrate a commitment to collaborate and local production networks in manufacturing, distribution and consumption processes. Sustainable fashion MSEs favor local and ethical production, quality, smaller collections and production runs, prefer a less demanding schedule, work outside of the traditional fashion calendar and they seek to reduce unnecessary waste. MSEs emphasize quality, durability/longevity, timelessness and creativity, collaboration, independence, flexibility, are value orientated, are transparent and believe in ethical practices. This results in innovative, independent, responsible fashion brands.

But sustainable fashion MSEs still face many challenges with respect to costs, pricing, access to sustainable resources (current innovation, verified information, financing) and communicating the value of sustainable fashion. They need targeted investment to strengthen the economic development potential of locally producing MSE responsible fashion brands. In the end the offer needs to be adopted by the customer and perceived as something valuable and desirable. The reDesign canvas attempts to provide these sustainable fashion MSE design entrepreneurs with a tool that addresses how to build sustainability in a holistic manner, utilizing 
systems thinking. The tool considers the relationship between sustainable business models and sustainable design practices and encourages designers to think of the five dimensions of sustainability proposed. Many of these sustainable fashion MSEs intuitively approach fashion through these five dimensions. The design entrepreneurs in this study are addressing all of the dimensions of sustainability, albeit to different degrees. What sustainable fashion MSE fashion design entrepreneurs contribute to the creation of a responsible fashion system is where and how they concentrate their efforts for sustainability. They demonstrate a commitment to creating a culture of sustainability, community engagement and fair and ethical working standards. They implement design strategies for aesthetic sustainability as they invest into new ways of operating fashion businesses and are dedicated to reducing environmental impacts through design. Their approach is holistic as they see sustainability as a lifestyle, not just supply chain optimization and reductions of negative impacts to be quantified in CSR reports. They have built businesses addressing the problems of the fashion industry. Specifically, the waste generated through the overproduction and overconsumption of fashion clothing. Their business and design practices are built around their beliefs and solutions to the specific problems they are tackling. The characteristic of the sustainable fashion MSEs in this study and their sustainable practices can be summarized in five main points:

1. They begin with a "problem" and the focus of their business is on solving that particular problem. Sustainable fashion MSEs demonstrate a start small and build approach as tackling all issues within sustainable fashion at the beginning is impossible.

2. The "problem" resonates with their beliefs and value systems.

3. Their business and design practices are built around those beliefs and solutions to the identified "problem".

4. They favor local and ethical production practices, high quality, smaller production runs to reduce overproduction and consumption and follow an anti-fashion calendar model (moving away from traditional "seasonal" fashion calendar).

5. They seek to promote responsible consumer behaviours, lifestyle changes and creating a culture of sustainability through authentic engagement.

To address the research question of this dissertation, How do sustainable fashion MSEs contribute to the creation of a responsible fashion system?, can be summarized in four points: 
1. Sustainable fashion MSE design entrepreneurs build and create a culture of sustainability. This is demonstrated through their design choices, their business model and stakeholder engagement where they seek to collaborate for the social and environmental well-being.

2. They recognize that waste through overproduction and overconsumption of fashion clothing is the one of fashion's industry biggest issues. As a result, they are actively finding solutions to combat the excessive waste through creative design, collaboration and sustainable business models.

3. These design entrepreneurs work outside the current structure and business practices that dominate the industry. This is executed through the use of anti-fashion calendar models, which promotes different approaches to how clothing is designed, manufactured and sold. For example, designing capsule collections that are released every three months and build upon the previous release as opposed to following the traditional seasonal calendar of spring/summer and fall/winter releases.

4. The sustainable fashion MSEs in this study predominately produce locally. MSEs that produce overseas source and produce from the same region, choosing to work with other small-scale producers. They are supporting local production networks where they can collaboratively work with their supply chain to support transparency, living wages and fair and ethical work practices.

However, they still face many challenges and future research should seek solutions to the challenges faced by sustainable fashion MSEs. The following are recommendations for potential solutions to the challenges and barriers faced by sustainable fashion MSEs:

1. Support small to medium sized producers who can work with sustainable fashion MSEs;

2. Build stronger local production networks based on collaboration for sustainability and innovation;

3. Ensure industry collaboration across various stakeholder groups to facilitate sharing of experiences, ideas and solutions for sustainable fashion (incubators are one means of achieving this);

4. Find ways to connect sustainable fashion MSEs to new sustainable technologies and innovations; 
5. Invest into producers to facilitate better communications and new sustainable technologies (as sustainable fashion MSEs do not have the means or influence to achieve this with producers).

Limitations of this dissertation are specific to the individual manuscripts. For manuscript two, Tools for Sustainable Fashion Design: An Analysis of their Fitness for Purpose, it was not possible to obtain the most current version of all tools. For example, the Higg Index requires a paid membership through an approval process that was not possible to obtain for this study. Therefore, a beta version which was available for free use to obtain feedback was the version used.

Manuscripts three and four, A Case Study of Sustainable Fashion MSEs and their Sustainable Design Practices and The reDesign Canvas: Fashion Design as a Tool for Sustainability, were qualitative case studies. Qualitative case study research is often faulted for issues with reliability and validity due to a lack of rigor and bias that is introduced due to the subjectivity of the researcher (Kirk \& Miller, 1986; Stoecker, 1991). The limitations of these manuscripts are directly related to their qualitative nature. The participants were selected for their sustainability-orientated beliefs and activities thus reducing generalizability to nonsustainable fashion businesses. This limitation provides future opportunity to explore how sustainable fashion design strategies employed by sustainable MSEs could translate and be adopted by non-sustainable fashion brands. As mentioned within the fourth manuscript, the reDesign canvas would benefit from further testing and iterations to improve its fitness for purpose for sustainable fashion MSEs.

Future directions for research should therefore investigate novel approaches to strengthen and grow local production networks to provide sustainable fashion MSEs with the resources and services needed. How can we cultivate hyperlocal communities based on responsibility, ethics, collaboration and innovation within fashion? There needs to be more support and growth of small to medium sized production networks that can support the specific needs of these sustainable fashion MSEs in terms of manageable MOQ's, small production runs and developing sustainable production methods. This entails investing into producers as well and enable them with better communication technology and new sustainable innovations and technologies for producing, recapturing and recycling clothing. This also includes finding ways to connect sustainable fashion MSEs to new sustainable technologies and innovations that support a circular 
economy. There needs to be industry collaboration across all stakeholder groups for a responsible fashion system. A facilitation of sharing experiences, ideas and solutions from all stakeholder groups, to diversify approaches and perspectives to include indigenous and marginalized voices - not just the dominate Western perspective of sustainability.

Design is an act of future-making (Simon, 1996; Yelavich \& Adams, 2014). Our realities are designed, and design will continue to create our future. For the future of fashion, sustainable choices will have to be an easily available alternative for designers and consumers. Designs needs to be considerate, responsible, desirable and stylish. Companies need to create trust by showing transparent and honest actions throughout their supply chains. Research into new technologies and innovation are required and must be made accessible for all to use. Collaboration is the only way forward to tackle the complexity we have designed into fashion. And for the consumers, knowledge needs to be provided and the meaning of sustainable fashion has to change. The new meaning of buying responsible fashion will have to be perceived as desirable and be related to how products and services in this sector are doing good for consumers, citizens, overall societies and the whole planet.

This dissertation undertook novel research into the sustainable design practices of MSEs and existing sustainable design tools. The hope is for other researchers to build upon this body of work, heed the recommendations for further tool development and begin to put the systems in place to better support the sustainable fashion MSEs working in the sustainable fashion space. This includes how to establish and support new sustainable business models, repositioning sustainable design entrepreneurs of MSEs as major contributors to the Canadian creative economy and drivers for a sustainable economy, and informing future policy development for sustainable creative industries. 


\section{REFERENCES}

Aakko, M., \& Koskennurmi-Sivonen, R. (2013). Designing sustainable fashion: Possibilities and challenges. Research Journal of Textile and Apparel, 17(1), 13-22. https://doi.org/10.1108/RJTA-17-01-2013-B002

Abidin, C. (2016). Visibility labour: Engaging with Influencers' fashion brands and\# OOTD advertorial campaigns on Instagram. ,. Media International Australia, 161(1), 86-100.

Adorno, T. W. (1991). The culture industry: Selected essays on mass culture. New York, NY: Routledge.

Agency [Def. 2.1]. (n.d.). Retrieved from https://en.oxforddictionaries.com/definition/agency

Allwood, J. M., Laursen, S. E., Russell, S. N., de Rodríguez, C. M., \& Bocken, N. M. P. (2008). An approach to scenario analysis of the sustainability of an industrial sector applied to clothing and textiles in the UK. Journal of Cleaner Production, 16(12), 1234-1246.

Almila, A. M. (2016). Cultural sociology of fashion: On the sartorial, symbolic and social. In D. Inglis \& A. M. Almila (Eds.), The SAGE Handbook of Cultural Sociology (pp. 510-526). London, UK: Sage.

Armstrong, C. M., \& LeHew, M. L. A. (2011). Sustainable apparel product development: In search of a new dominant social paradigm for the field using sustainable approaches. Fashion Practice: The Journal of Design, Creative Process \& the Fashion Industry, 3(1), $29-62$.

Au, J. S., Taylor, G., \& Newton, E. W. (2001). A comparative analysis of grounded design theories of European and Japanese fashion designers. Fashion \& Textile Research Journal, $3(5), 444-454$.

Baldassarre, B., Calabretta, G., Bocken, N. M. P., \& Jaskiewicz, T. (2017). Bridging sustainable business model innovation and user-driven innovation: A process for sustainable value proposition design. Journal of Cleaner Production, 147, 175-186.

Balkenende, R., \& Bakker, C. (2018). Designing for a Circular Economy: Make, Use and Recover Products. In K. Niinimäki (Ed.), Sustainable Fashion in a Circular Economy (pp. 76-95). Helsinki, Finland: Aalto University.

Bansal, P., \& Song, H.-C. (2017). Similar but not the same: Differentiating corporate 
sustainability from corporate responsibility. Academy of Management Annals, 11(1), 105149.

Barnard, M. (2014). Fashion theory: An introduction. London, UK: Routledge.

Barnes, L. (2013). Fashion marketing. Textile Progress, 45(2-3), 182-2007.

Barron, L. (2007). The habitus of Elizabeth Hurley: Celebrity, fashion, and identity branding. Fashion Theory, 11(4), 443-461.

Battaglia, M., Testa, F., Bianchi, L., Iraldo, F., \& Frey, M. (2014). Corporate social responsibility and competitiveness within SMEs of the fashion industry: Evidence from Italy and France. Sustainability (Switzerland), 6(2), 872-893. https://doi.org/10.3390/su6020872

Baumann, H., Berlin, J., Brunklaus, B., Lindkvist, M., Löfgren, B., \& Tillman, A.-M. (2011). The usefulness of an actor's perspective in LCA. In M. Finkbeiner (Ed.), Towards Life Cycle Sustainability Management (pp. 73-83). Berlin: Springer Verlag. https://doi.org/10.1007/978-94-007-1899-9

Beatty, S. E., \& Kahle, L. R. (1988). Alternative hierarchies of the attitude-behavior relationship: The impact of brand commitment and habit. Journal of the Academy of Marketing Science, 16(2), 1-10.

Bhardwaj, V., \& Fairhurst, A. (2010). Fast fashion: response to changes in the fashion industry. The International Review of Retail, Distribution and Consumer Research, 20(1), 165-173.

Birtwistle, G., \& Moore, C. M. (2007). Fashion clothing-where does it all end up? International Journal of Retail \& Distribution Management, 35(3), 210-216.

Black, S. (2012). The Sustainable fashion handbook. London, UK: Thames \& Hudson.

Black, S., \& Eckert, C. (2012). Considerate design: Supporting sustainable fashion design. In S. Black (Ed.), The Sustainable Fashion Handbook (pp. 92-95). London, UK: Thames \& Hudson.

Bluesign. (2016). Brands. Retrieved from http://www.bluesign.com/industry/brands\#.V_GiH5MrJE5

Bocken, N M P, Farracho, M., Bosworth, R., \& Kemp, R. (2014). The front-end of ecoinnovation for eco-innovative small and medium sized companies. Journal of Engineering 
and Technology Management, 31, 43-57.

Bocken, N M P, Short, S. W., Rana, P., \& Evans, S. (2014). A literature and practice review to develop sustainable business model archetypes. Journal of Cleaner Production, 65, 42-56. https://doi.org/10.1016/j.jclepro.2013.11.039

Bocken, Nancy M P, de Pauw, I., Bakker, C., \& van der Grinten, B. (2016). Product design and business model strategies for a circular economy. Journal of Industrial and Production Engineering, 33(5), 308-320.

Boons, F., Montalvo, C., Quist, J., \& Wagner, M. (2013). Sustainable innovation, business models and economic performance: an overview. Journal of Cleaner Production, 45, 1-8.

Boons, Frank, \& Lüdeke-Freund, F. (2013). Business models for sustainable innovation: stateof-the-art and steps towards a research agenda. Journal of Cleaner Production, 45, 9-19.

Borukhovich, B. (2009). Fashion design: The work of art that is still unrecognized in the United States. Wake Forest Intellectual Property Law Journal, 9, 155-238.

Bos-Brouwers, H. E. J. (2010). Corporate sustainability and innovation in SMEs: evidence of themes and activities in practice. Business Strategy and the Environment, 19(7), 417-435.

Boucher, J., \& Friot, D. (2017). Primary microplastics in the oceans: A global evaluation of sources. International Union for Conservation of Nature. https://doi.org/10.2305/iucn.ch.2017.01.en

Bourdieu, P. (1993a). Sociology in Question. London, UK: Sage Publications Inc.

Bourdieu, P. (1993b). The field of cultural production: Essays on art and literature. New York, NY: Columbia University Press.

Bourdieu, P. (1996). The rules of art: Genesis and structure of the literary field (S. Emanuel, Trans.). Stanford, CA: Standford University Press.

Bourdieu, P., \& Delsaut, Y. (1975). Le couturier et sa griffe: contribution à une théorie de la magie. Actes de La Recherche En Sciences Sociales, 1(1), 7-36.

Bourdieu, P. (1986). The forms of capital. In J. G. Richardson (Ed.), Handbook of Theory and Research for the Sociology of Education (pp. 241-258). New York, NY: Greenwood.

Bourdieu, Pierre. (1977). Outline of a Theory of Practice (Vol. 16). Cambridge university press. 
Bourdieu, Pierre, \& Wacquant, L. J. D. (1992). An invitation to reflexive sociology. University of Chicago press.

Bovea, M. D., \& Pérez-Belis, V. (2012). A taxonomy of ecodesign tools for integrating environmental requirements into the product design process. Journal of Cleaner Production, 20(1), 61-71. https://doi.org/10.1016/j.jclepro.2011.07.012

Braun, V., \& Clarke, V. (2006). Using thematic analysis in psychology. Qualitative Research in Psychology, 3(2), 77-101.

Braun, V., \& Clarke, V. (2019). Thematic analysis. In P. Liamputtong (Ed.), Handbook of Research Methods in Health Social Sciences (pp. 843-860). Singapore: Springer.

Brezet, H. (1997). Dynamics in Ecodesign Practice. UNEP Industry and Environment, 20(1-2), 21-24.

Briggs, A. (2013). Capitalism's favorite child: The production of fashion. In S. Bruzzi \& P. Church Gibson (Eds.), Fashion cultures revisted. New York, NY: Routledge.

Brockman, H. L. (1965). The theory of fashion design. New York, NY: Wiley.

Brooks, A. (2015). Clothing Poverty. London, UK: Zed Books.

Brown, T. (2008). Design thinking. Harvard Business Review, 86(6), 86-87.

Brown, T., \& Wyatt, J. (2010). Design thinking for social innovation IDEO. Development Outreach, 12(1), 29-43. https://doi.org/10.1596/1020-797X_12_1_29

Buchanan, R. (1992). Wicked problems in design thinking. Design Issues, 8(2), 5-21.

Caniato, F., Caridi, M., Crippa, L., \& Moretto, A. (2012). Int . J . Production Economics Environmental sustainability in fashion supply chains : An exploratory case based research. Intern. Journal of Production Economics, 135(2), 659-670. https://doi.org/10.1016/j.ijpe.2011.06.001

Carbon Disclosure Project. (2019). About us. https://doi.org/https://www.cdp.net/en/info/aboutus

Cataldi, C., Dickson, M., \& Grover, C. (2010). Slow fashion: tailoring a strategic approach towards sustainability.

Centre for Sustainable Fashion. (2013). Making:Nike. Retrieved from http://sustainable- 
fashion.com/projects/nike/

Ceschin, F., \& Gaziulusoy, I. (2016). Evolution of design for sustainability: From product design to design for system innovations and transitions. Design Studies, 47, 118-163. https://doi.org/10.1016/j.destud.2016.09.002

Chan, T., \& Wong, C. W. Y. (2012). The consumption side of sustainable fashion supply chain: Understanding fashion consumer eco-fashion consumption decision. Journal of Fashion Marketing and Management, 16(2), 193-215.

Chapman, J. (2005). Emotionally durable design: objects, experiences and empathy. London, UK: Earthscan.

Chapman, J. (2009). Design for (emotional) durability. Design Issues, 25(4), 29-35.

Chapman, J. (2014). Prospect, seed and activate: Advancing design for sustainability in fashion.

In Kate Fletcher \& M. Tham (Eds.), Routledge handbook of sustainability and fashion (pp. 74-81). New York, NY: Routledge.

Choi, K. H., \& Lewis, V. D. (2018). An inclusive system for fashion criticism. International Journal of Fashion Design, Technology and Education, 11(1), 12-21. https://doi.org/http://dx.doi.org/10.1080/17543266.2017.1284272

Clark, H. (2008). SLOW+ FASHION—an Oxymoron-or a Promise for the Future...? Fashion Theory: The Journal of Dress, Body \& Culture, 12(4), 427-446.

Collins-Dodd, C., \& Lindley, T. (2003). Store brands and retail differentiation: the influence of store image and store brand attitude on store own brand perceptions. Journal of Retailing and Consumer Services, 10(6), 345-352.

Connor-Crabb, A. C. (2017). Fashion Design for Longevity: Design strategies and their implementation in practice. University of Brighton.

Cope, M. (2005). Coding qualitative data. In I. Hay (Ed.), Qualitative research methods in human geography (pp. 223-233). New York, NY: Oxford University Press.

Cradle to Cradle Products Innovation Institute. (2018). Cradle 2 Cradle certified products program. Retrieved from https://www.c2ccertified.org/

Craik, J. (2009). Fashion: The key concepts. Oxford, UK: Berg.

Çukul, D. (2015). Fashion marketing in social media: using Instagram for fashion branding. In 
Proceedings of 1st Business and Management Conferences (pp. 116-129). Vienna:

International Institute of Social and Economic Sciences.

Dawar, N., \& Parker, P. (1994). Marketing universals: Consumers' use of brand name, price, physical appearance, and retailer reputation as signals of product quality. Journal of Marketing, 58(2), 81-95.

De Brito, M. P., Carbone, V., \& Blanquart, C. M. (2008). Towards a sustainable fashion retail supply chain in Europe: organisation and performance. International Journal of Production Economics, 114(2), 534-553.

De Bruycker, J. (2016). The future of branding is debranding. Fast Company. Fast Company.

Retrieved from https://www.fastcodesign.com/3060658/the-future-of-branding-isdebranding

De Medeiros, J. F., Ribeiro, J. L. D., \& Cortimiglia, M. N. (2014). Success factors for environmentally sustainable product innovation: a systematic literature review. Journal of Cleaner Production, 65, 76-86. https://doi.org/10.1016/j.jclepro.2013.08.035

Deaton, A., \& Muellbauer, J. (1980). Economics and consumer behavior. Cambridge, UK: Cambridge University Press.

DeLong, M., Goncu-Berk, G., Bye, E., \& Wu, J. (2016). Apparel Sustainability from a Local Perspective. Research Journal of Textile and Apparel, 17(1), 59-69. https://doi.org/10.1108/rjta-17-01-2013-b006

Design School Kolding. (2018a). Approaches to Sustainable Design. Retrieved from https://sustainabledesigncards.dk/

Design School Kolding. (2018b). Sustainable Design Cards. Retrieved from https://sustainabledesigncards.dk/

Dholakia, U. M. (1997). An investigation of some determinants of brand commitment. Advances in Consumer Research, 24(1), 381-387.

DiCicco-Bloom, B., \& Crabtree, B. F. (2006). The qualitative research interview. Medical Education, 40(4), 314-321.

Dick, A., \& Basu, K. (1994). Customer loyalty: Toward an integrated conceptual framework. Journal of the Academy of Marketing Science, 22, 99-113. 
Dimitriadis, N. I., \& Koh, S. C. L. (2005). Information flow and supply chain management in local production networks: the role of people and information systems. Production Planning \& Control, 16(6), 545-554. https://doi.org/10.1080/09537280500112397

Doherty, A. M. (1999). Explaining international retailers' market entry mode strategy: Internalization theory, agency theory and the importance of information asymmetry. International Review of Retail, Distribution and Consumer Research, 9(4), 379-402.

Dorst, K. (2011). The core of 'design thinking'and its application. Design Studies, 32(6), 521532.

Duhigg, C. (2012). The Power of Habit: Why we do what we do in life and business. Canada: Anchor Canada.

Earley, R. (2017). Designing Fast \& Slow. Exploring fashion textile product lifecycle speeds with industry designers. The Design Journal, 20(sup1), S2645-S2656. https://doi.org/10.1080/14606925.2017.1352776

Easterbrook, J. A. (1959). The effect of emotion on cue utilization and the organization of behavior. Psychological Review, 66, 183-201.

Eckert, C. (2001). The communication bottleneck in knitwear design: Analysis and computing solutions. Computer Supported Cooperative Work, 10(1), 29-74.

Eckman, M., \& Wagner, J. (1995). Aesthetic aspects of the consumption of fashion design: The conceptual and empirical challenge. Advances in Consumer Research, 22, 646-649.

Elkington, J. (1997). Cannibals with forks: The triple bottom line of 21 st century business. Oxford, UK: Capstone.

Ellen MacArthur Foundation. (2017). A new textiles economy: redesigning fashion's future. Ellen MacArthur Foundation. Retrieved from https://www.ellenmacarthurfoundation.org/publications/a-new-textiles-economyredesigning-fashions-future

Ellen MacArthur Foundation. (2019). Artificial intelligence and the circular economy - AI as a tool to accelerate the transition. Retrieved from https://www.ellenmacarthurfoundation.org/assets/downloads/Artificial-intelligence-and-thecircular-economy.pdf 
Entwistle, J. (2000). The fashioned body: Fashion, dress and modern social theory. Cambridge: Routledge.

Entwistle, J. (2002). The aesthetic economy: The production of value in the field of fashion modelling. Journal of Consumer Culture, 2(3), 317-339.

Entwistle, J. (2009). The aesthetic economy of fashion: Markets and values in clothing and modelling. Oxford: Berg.

Entwistle, J., \& Rocamora, A. (2006). The field of fashion materialized: a study of London Fashion Week. Sociology, 40(4), 735-751.

Erk, S., Spitzer, M., Wunderlich, A. P., Galley, L., \& Walter, H. (2002). Cultural objects modulate reward circuitry. Neuroreport, 13(18), 2499-2503.

Esslinger, H. (2011). Sustainable Design: Beyond the Innovation-Driven Business Model. Journal of Product Innovation Management, 28(3), 401-404.

Evans, C. (2003). Fashion at the edge: spectacle, modernity and deathliness. New Haven, CT: Yale University Press.

Evans, D. (2011). System of provision. In D. Southerton (Ed.), Encyclopedia of Consumer Culture. Thousand Oaks, CA: SAGE.

Fashion for Good. (2018). Fashion for Good. Retrieved from https://fashionforgood.com/

Fashion Revolution. (2019). Fashion Revolution. Retrieved from https://www.fashionrevolution.org/

Fine, B. (2002). The world of consumption: The material and cultural revisted. London, UK: Routledge.

Fine, B., \& Leopold, E. (1993). The world of consumption. London, UK: Routledge.

Finlay, K. A., Trafimow, D., \& Villarreal, A. (2002). Predicting Exercise and Health Behavioral Intentions: Attitudes, Subjective Norms, and Other Behavioral Determinants1. Journal of Applied Social Psychology, 32(2), 342-356.

Fishbein, M., \& Ajzen, I. (1975). Belief, attitude, intention, and behavior: An introduction to theory and research. Reading, MA: Addison-Wesley.

Fletcher, K. (2008). Sustainable fashion \& textiles: Design journeys. London, UK: Earthscan. 
Fletcher, K. (2010). Slow fashion: an invitation for systems change. Fashion Practice: The Journal of Design, Creative Process \& the Fashion Industry, 2(2), 259-266.

Fletcher, K, Grose, L., \& Hawken, P. (2012). Fashion and Sustainability: Design for Change. London, UK: Laurence King. Retrieved from https://books.google.ca/books?id=kFZAXwAACAAJ

Fletcher, Kate. (2012). Durability, Fashion, Sustainability: The Processes and Practices of Use. Fashion Practice: The Journal of Design, Creative Process \& the Fashion, 4(2), 221-238. https://doi.org/10.2752/175693812X13403765252389

Flood Heaton, R., \& McDonagh, D. (2017). Can Timelessness through Prototypicality Support Sustainability? A Strategy for Product Designers. The Design Journal, 20(sup1), S110S121. https://doi.org/10.1080/14606925.2017.1352671

Friedman, J. (1994). Consumption and identity. Amsterdamn, The Netherlands: Overseas Publishers Association.

Fuad-Luke, A. (2010). Adjusting our metabolism: Slowness and nourishing rituals of delay in anticipation of a post-consumer age. In T. Cooper (Ed.), Longer lasting products: Alternatives to the throwaway society (pp. 133-155). Farnham, UK: Gower.

Georgiou, I. (2007). Thinking through systems thinking. New York, NY: Routledge.

Ghisellini, P., Cialani, C., \& Ulgiati, S. (2016). A review on circular economy: the expected transition to a balanced interplay of environmental and economic systems. Journal of Cleaner Production, 114, 11-32.

Gilbert, D. (2017). The Looks of Austerity: Fashions for Hard Times. Fashion Theory - Journal of Dress Body and Culture, 21(4), 477-499. https://doi.org/10.1080/1362704X.2017.1316057

Global Fashion Agenda. (2019). CEO Agenda 2019. Retrieved from https://www.globalfashionagenda.com/ceo-agenda-2019/\#

Global Fashion Agenda, \& Boston Consulting Group. (2017). Pulse of the Fashion Industry Report. Retrieved from https://www.copenhagenfashionsummit.com/wpcontent/uploads/2017/05/Pulse-of-the-Fashion-Industry_2017.pdf

Global Reporting Initiative. (2019). Apparel and footwear. Retrieved from 
https://www.globalreporting.org/information/sector-guidance/sector-guidance/pilotversions/apparel-and-footwear/Pages/default.aspx

Goldsmith, R. E., Freiden, J. B., \& Kilsheimer, J. C. (1993). Social values and female fashion leadership: A cross-cultural study. Psychology \& Marketing, 10(5), 399-412.

GOTS. (2016). The standard. Retrieved from http://www.global-standard.org/the-standard.html Greenpeace. (2019). Detox my fashion. Retrieved from https://www.greenpeace.org/international/act/detox/

Greenwood, R., \& Suddaby, R. (2006). Institutional entrepreneurship in mature fields: The big five accounting firms. Academy of Management Journal, 49(1), 27-48.

Grinberg, E. (2013). Style bloggers to follow in any season. Retrieved from http://www.cnn.com/2013/02/20/living/nyfw-fashion-bloggers/index.html

Guest, G., \& McLellan, E. (2003). Distinguishing the Trees from the Forest : Field Methods, 15(2), 186-201. https://doi.org/10.1177/1525822X03251188

Guidice, F., La Rose, G., \& Risitano, A. (2006). Product Design for the Environment: A Life Cycle Approach. Boca Raton, FL: Taylor \& Francis.

Gwilt, A. (2015). Exploring a Framework for Fashion Design for Sustainability. In S. S. Muthu (Ed.), Handbook of Sustainable Apparel Production (pp. 439-452). Boca Raton, FL: CRC Press.

Gwilt, A., \& Rissanen, T. (2011). Shaping sustainable fashion: Changing the way we make and use clothes. London, UK: Earthscan.

Gwozdz, W., Steensen Nielsen, K., \& Müller, T. (2017). An environmental perspective on clothing consumption: Consumer segments and their behavioral patterns. Sustainability, $9(5), 762$.

Hahn, K. H. Y., \& Lee, E.-J. (2014). Effect of psychological closeness on consumer attitudes toward fashion blogs: the moderating effect of fashion leadership and interpersonal LOV. Journal of Global Fashion Marketing, 5(2), 103-121.

Hall, J. K., Daneke, G. A., \& Lenox, M. J. (2010). Sustainable development and entrepreneurship: Past contributions and future directions. Journal of Business Venturing, 25(5), 439-448. 
Harper, K. (2018). aesthetic sustainability: product design and sustainable usage. Abington, Oxon: Routledge.

Harrington, A. (2005). Modern social theory. Oxford, UK: Oxford University Press.

Harris, J. (1995). Costume history and fashion theory: Never the twain shall meet? Bulletin of the John Rylands University Library of Manchester, 77(1), 73-79.

Harvey, L., \& Green, D. (1993). Defining quality. Assessment \& Evaluation in Higher Education, 18(1), 9-34.

Hasling, K. M., \& Ræbild, U. (2017). Sustainability Cards: Design for Longevity. In Product Lifetimes and the Environment (pp. 166-170). Delft University of Technology. Retrieved from https://adk.elsevierpure.com/ws/portalfiles/portal/61261451/Hasling_R_bild_2017_Sustaina bility_Cards.pdf

Hauge, A., Malmberg, A., \& Power, D. (2009). The spaces and places of Swedish fashion. European Planning Studies, 17(4), 529-547.

Henninger, C. E., Alevizou, P. J., \& Oates, C. J. (2016). What is sustainable fashion? Journal of Fashion Marketing and Management: An International Journal, 20(4), 400-416. https://doi.org/10.1108/JFMM-07-2015-0052

Hesmondhalgh, D. (2006). Bourdieu, the media and cultural production. Media, Culture and Society, 28(2), 211-231.

Hethorn, J., \& Ulasewicz, C. (2008). Sustainable fashion: Why now. New York, NY: Fairchild.

Hoffman, E. (2012). User integration in sustainable product development. Sheffield, UK: Greenleaf.

Hollander, A. (1992). The modernization of fashion. Design Quarterly, 154, 27-33.

Horn, M. J., \& Gurel, L. M. (1975). The second skin: An interdisciplinary study of clothing. Wilmington, MA: Houghton Mifflin.

Hsieh, H.-F., \& Shannon, S. E. (2005). Three approaches to qualitative content analysis. Qualitative Health Research, 15(9), 1277-1288.

Hu, Z. H., Li, Q., Chen, X. J., \& Wang, Y. F. (2014). Sustainable rent-based closed-loop supply chain for fashion products. Sustainability, 6(10), 7063-7088. 
https://doi.org/http://doi:10.3390/su6107063

Hur, E., Beverley, K., \& Cassidy, T. (2013). Development of An Ideation Toolkit Supporting Sustainable Fashion Design and Consumption. Research Journal of Textile and Apparel, 17(2), 89-100. https://doi.org/https:// doi.org/10.1108/RJTA-17-02-2013-B012

Hur, E., \& Cassidy, T. (2019). Perceptions and attitudes towards sustainable fashion design: challenges and opportunities for implementing sustainability in fashion. International Journal of Fashion Design, Technology and Education, 0(0), 1-10. https://doi.org/10.1080/17543266.2019.1572789

Instagram. (2016). Instagram Advertiser Numbers. Instagram for Business. Retrieved from http://blog.business.instagram.com/post/150771324916/instagram-advertiser-number

Instagram. (2017). Press News. Retrieved from https://instagram-press.com/

Jarvis, A. (1998). Letter from the editor. Fashion Theory, 2(4), 299-300.

Jenkins, H. (2009). A ‘business opportunity’model of corporate social responsibility for smalland medium-sized enterprises. Business Ethics: A European Review, 18(1), 21-36.

Jenkyn Jones, S. (2011). Fashion Design. London, UK: Laurence King Publishing.

Jestratijevic, I., \& Rudd, N. A. (2018). Six Forms of Sustainable Fashion. Latest Trends in Textile and Fashion Designing, 2(4), 220-222. https://doi.org/10.32474/LTTFD.2018.02.000145

Ji, M. F., \& Wood, W. (2007). Purchase and consumption habits: Not necessarily what you intend. Journal of Consumer Psychology, 17(4), 261-276.

Jin Gam, H., Cao, H., Farr, C., \& Heine, L. (2009). C2CAD: A sustainable apparel design and production model. International Journal of Clothing Science and Technology, 21(4), 166179. https://doi.org/10.1108/09556220910959954

Jung, S., \& Jin, B. (2014). A theoretical investigation of slow fashion: Sustainable future of the apparel industry. International Journal of Consumer Studies, 38(5), 510-519. https://doi.org/10.1111/ijcs.12127

Kaiser, S. B., Nagasawa, R. H., \& Hutton, S. S. (1989). Construction of an SI Theory of Fashion : Part 2 . From Discovery to Formalization. Clothing and Textiles Research Journal, (3), 234-244. 
Kaiser, S. B., Nagasawa, R. H., \& Hutton, S. S. (1995). Construction of An SI Theory of Fashion: Part 1. Ambivalence and Change. Clothing and Textiles Research Journal, 13(3), 172-183. https://doi.org/10.1177/0887302X9501300304

Karaosman, H., Morales-Alonso, G., \& Brun, A. (2016). From a systematic literature review to a classification framework: Sustainability integration in fashion operations. Sustainability, 9(1), 30. https://doi.org/http://doi:10.3390/su9010030

Karell, E. (2013). Planned continuity - Multi-life garments through modular structures and supplemental services. In K. Niinimaki (Ed.), Sustainable fashion: New approaches (pp. 110-125). Helsinki, Finland: Aalto University.

Keller, K. (2001). Building customer-based brand equity: A blueprint for creating strong brands. Marketing Management, 10(July/August), 15-19.

Kimbell, L. (2011). Rethinking design thinking: Part I. Design and Culture, 3(3), 285-306.

Kirchain, R., Olivetti, E., Miller, T. R., \& Greene, S. (2015). Sustainable Apparel Materials: An overview of what we know and what could be done about the impact of four major apparel materials: Cotton, Polyester, Leather, \& Rubber. MIT. Cambridge, MA. https://doi.org/10.3390/resources3010319

Kirk, J. L., \& Miller, M. (1986). Reliability and validity in qualitative research. Beverly Hills, CA: Sage.

Knight, P., \& Jenkins, J. O. (2009). Adopting and applying eco-design techniques: A practitioners perspective. Journal of Cleaner Production, 17(5), 549-558.

Kozlowski, A., Searcy, C., \& Bardecki, M. (2015). Corporate sustainability reporting in the apparel industry an analysis of indicators disclosed. International Journal of Productivity and Performance Management, 64(3). https://doi.org/10.1108/IJPPM-10-2014-0152

Kozlowski, Anika, Bardecki, M., \& Searcy, C. (2019). Tools for sustainable fashion design: An analysis of their fitness for purpose. Sustainability, 11(13), 3581. https://doi.org/10.3390/su11133581

Kozlowski, Anika, Searcy, C., \& Bardecki, M. (2018). The reDesign canvas: Fashion design as a tool for sustainability. Journal of Cleaner Production, 183(10), 194-207. https://doi.org/10.1016/j.jclepro.2018.02.014 
Krippendorff, K. (2006). The Semantic Turn: A new foundation for design. Boca Raton, FL: CRC Press.

Laitala, K., Boks, C., \& Klepp, I. G. (2015). Making clothing last: A design approach for reducing the environmental impacts. International Journal of Design, 9(2), 93-107.

Lakhal, S. Y., Sidibé, H., \& H'Mida, S. (2008). Comparing conventional and certified organic cotton supply chains: the case of Mali. International Journal of Agricultural Resources, Governance and Ecology, 7(3), 243-255.

Lally, P., Van Jaarsveld, C. H. M., Potts, H. W. W., \& Wardle, J. (2010). How are habits formed: Modelling habit formation in the real world. European Journal of Social Psychology, 40(6), 998-1009.

Lamb, J. M., \& Kallal, M. J. (1992). A conceptual framework for apparel design. Clothing and Textiles Research Journal, 10(2), 42-47.

LaPiere, R. T. (1934). Attitudes vs. actions. Social Forces, 13(2), 230-237.

Lavrakas, P. J. (2011). Encyclopedia of Survey Research Methods. Thousand Oaks, CA: Sage Publications Inc. https://doi.org/10.4135/9781412963947

Lawless, E., \& Medvedev, K. (2016). Assessment of sustainable design practices in the fashion industry: Experiences of eight small sustainable design companies in the Northeastern and Southeastern United States. International Journal of Fashion Design, Technology and Education, 9(1), 41-50. https://doi.org/10.1080/17543266.2015.1116616

Lemmerer, A., \& Menrad, K. (2017). Attitudes and habit in value perception for foods. Journal of Food Products Marketing, 23(2), 99-121.

Leopold, E. (1993). The manufacture of the fashion system. In J. Ash \& E. Wilson (Eds.), Chic thrills: A fashion reader (pp. 101-117). Berkeley, CA: University of California Press.

Liu-Thompkins, Y., \& Tam, L. (2013). Not all repeat customers are the same: Designing effective cross-selling promotion based on attitudinal loyalty and habit. Journal of Marketing, 77(5), 21-36.

Lofthouse, V. (2006). Ecodesign tools for designers: defining the requirements. Journal of Cleaner Production, 14(15-16), 1386-1395.

Loschek, I. (2009). When clothes become fashion: Design and innovative systems. Oxford, UK: 
Berg.

Lüdeke-Freund, F. (2010). Towards a Conceptual Framework of'Business Models for Sustainability'. ERSCP-EMU Conference. Delft, The Netherlands.

Lynch, A., \& Strauss, M. (2007). Changing fashion: A critical introduction to trend analysis and cultural meaning. Oxford, UK: Berg.

Mackrell, A. (2005). Fashion and art: The impact of art on fashion and fashion on art. London, UK: Batsford.

Made-By. (2018). MODE Tracker. Retrieved from http://www.made-by.org/modetracker/ Madge, P. (1993). Design, ecology, technology: A historiographical review. Journal of Design History, 6(3), 149-166.

Manzini, E. (2015). Design, When Everybody Designs: An Introduction to Design for Social Innovation. Cambridge MA: MIT Press.

Manzini, E., \& Vezzoli, C. (2002). Product-service systems and sustainability: Opportunities for sustainable solutions. Paris, France: UNEP.

Manzini, E., \& Vezzoli, C. (2003). A strategic design approach to develop sustainable product service systems: examples taken from the 'environmentally friendly innovation'Italian prize. Journal of Cleaner Production, 11(8), 851-857.

Marcus, J., Kurucz, E., \& Colbert, B. A. (2010). Conceptions of the business-society-nature interface: implications for management scholarship. Business \& Society, 49(3), 402-438.

Martin, R. (1999). A note: Art and Fashion, Viktor and Rolf. Fashion Theory, 3(1), 109-120.

May-Plumlee, T., \& Little, T. J. (1998). No-interval coherently phased product development model for apparel. International Journal of Clothing Science and Technology, 10(5), 342364.

McRobbie, A. (1998). British fashion design. London, UK: Routledge.

Meadows, D. (2008). Thinking in systems. White River Junction, VT: Chelsea Green .

Meadows, D. H. (1999). Leverage points: places to intervene in a system. Retrieved from http://donellameadows.org/archives/leverage-points-places-to-intervene-in-a-system/

Mentz, S. (2012). After Sustainability. PMLA, 127(3), 586-592. 
Mete, F. (2006). The creative role of sources of inspiration in clothing design. International Journal of Clothing Science and Technology, 18(4), 278-293.

Miller, C. M., McIntyre, S. H., \& Mantrala, M. K. (1993). Toward formalizing fashion theory. Journal of Marketing Research, 30(2), 142-157.

Min, H., \& Galle, W. P. (2001). Green purchasing practices of US firms. International Journal of Operations \& Production Management, 21(9), 1222-1238.

Minney, S. (2017). Slave to Fashion. Oxford, UK: New Internationalist Publications Ltd.

Montiel, I. (2008). Corporate social responsibility and corporate sustainability: Separate pasts, common futures. Organization \& Environment, 21(3), 245-269.

Mora, E., Rocamora, A., \& Volonté, P. (2014). On the issue of sustainability in fashion studies. International Journal of Fashion Studies, 1(2), 139-147. https://doi.org/10.1386/infs.1.2.139_1

Morgan, L. R., \& Birtwistle, G. (2009). An investigation of young fashion consumers' disposal habits. International Journal of Consumer Studies, 33(2), 190-198.

Nagasawa, R. H., Kaiser, S. B., \& Hutton, S. S. (1996). Construction of an SI Theory of Fashion: Part 3. Context of Explanation. Clothing and Textiles Research Journal, 14(1), 54-62. https://doi.org/10.1177/0887302x9601400107

Navarro, Z. (2006). In search of a cultural interpretation of power: the contribution of Pierre Bourdieu. IDS Bulletin, 37(6), 11-22.

Neal, D. T., Wood, W., Labrecque, J. S., \& Lally, P. (2012). How do habits guide behavior? Perceived and actual triggers of habits in daily life. Journal of Experimental Social Psychology, 48(2), 492-498.

Neal, D. T., Wood, W., \& Quinn, J. M. (2006). Habits-A repeat performance. Current Directions in Psychological Science, 15(4), 198-202.

Nicklas, C. (2014). One essential thing to learn is color: Harmony, science and color theory in mid-nineteenth century fashion advice. Journal of Design History, 27(3), 218-236.

Niinimäki, K. (2006). Ecodesign and textiles. Research Journal of Textile and Apparel, 10(3), $67-75$.

Niinimäki, K., \& Hassi, L. (2011). Emerging design strategies in sustainable production and 
consumption of textiles and clothing. Journal of Cleaner Production, 19(16), 1876-1883.

Nike Inc. (2015). Making of Making by NIKE MSI. Retrieved from

https://itunes.apple.com/us/app/making-making-powered-by-nike/id662227880

Norman, D. A. (2010). Design Thinking: A Useful Myth. Retrieved from

http://www.core77.com/posts/16790/design-thinking-a-useful-myth-16790

Norman, D. A. (2013). Rethinking design thinking. Retrieved from

http://www.core77.com/posts/24579/Rethinking-Design-Thinking

Nystrom, P. H. (1928). Economics offashion. New York, NY: The Ronald Press Company.

Retrieved from http://babel.hathitrust.org/cgi/pt?id=mdp.39015009354666;view=1up;seq=5

Oliver, R. L. (1999). Whence consumer loyalty? Journal of Marketing, 63(4), 33-44.

Osterwalder, Alex, Pigneur, Y., Bernarda, G., \& Smith, A. (2014). Value proposition design. Hoboken, NJ: Wiley.

Osterwalder, Alexander, \& Pigneur, Y. (2010). Business model generation: A handbook for visionaries, game changers, and challengers. Hoboken, NJ: Wiley.

Ozdamar Ertekin, Z., \& Atik, D. (2015). Sustainable Markets Motivating Factors, Barriers, and Remedies for Mobilization of Slow Fashion. Journal of Macromarketing, 35(1), 53-69.

Palomo-Lovinski, N., \& Hahn, K. (2014). Fashion Design Industry Impressions of Current Sustainable Practices. Fashion Practice: The Journal of Design, Creative Process \& the Fashion Industry, 6(1), 87-106.

Pan, B., \& Holland, R. (2006). A mass customised supply chain for the fashion system at the design-production interface. Journal of Fashion Marketing and Management: An International Journal, 10(3), 345-359.

Pannabecker, R. K. (1997). Fashioning theory: A critical discussion of the symbolic interactionist theory of fashion. Clothing and Textiles Research Journal, 15(3), 178-183.

Papanek, V. (1984). Design for the real world. London, UK: Thames \& Hudson.

Pedersen, E. R. G., \& Andersen, K. R. (2015). Sustainability innovators and anchor draggers: a global expert study on sustainable fashion. Journal of Fashion Marketing and Management, 19(3), 315-327. 
Pedroni, M. (2015). "Stumbling on the heels of my blog": Career, forms of capital, and strategies in the (sub) field of fashion blogging. Fashion Theory, 19(2), 179-199.

Pettinger, L. (2004). Brand culture and branded workers: Service work and aesthetic labour in fashion retail. Consumption Markets \& Culture, 7(2), 165-184.

Pitchipoo, P., Venkumar, P., \& Rajakarunakaran, S. (2015). Grey decision model for supplier evaluation and selection in process industry: a comparative perspective. The International Journal of Advanced Manufacturing Technology, 76(9-12), 2059-2069.

Pookulangara, S., \& Shephard, A. (2013). Slow fashion movement: Understanding consumer perceptions-An exploratory study. Journal of Retailing and Consumer Services, 20(2), 200-206.

Porter, T., \& Córdoba, J. (2009). Three views of systems theories and their implications for sustainability education. Journal of Management Education, 33(3), 323-347.

Pousman, Z., \& Stasko, J. (2006). A taxonomy of ambient information systems: Four patterns of design. In Proceedings of the working conference on Advanced visual interfaces - AVI '06 (pp. 67-74). https://doi.org/10.1145/1133265.1133277

Ræbild, U., \& Hasling, K. M. (2018). Sustainable Design Cards: A Learning Tool for Supporting Sustainable Design Strategies. In K. Niinimäki (Ed.), Sustainable Fashion in a Circular Economy (pp. 128-151). Espoo: Aalto University.

Ramani, K., Ramanujan, D., Bernstein, W. Z., Zhao, F., Sutherland, J., Handwerker, C., ... Thurston, D. (2010). Integrated sustainable life cycle design: A review. Journal of Mechanical Design, 132(9), 9100401-9100415. https://doi.org/10.1115/1.4002308

Reinach, S. (2005). China and Italy: Fast fashion versus pret_a porter. Towards a new culture of fashion. Fashion Theory, 9(1), 43-56.

Remy, N., Speelman, E., \& Swartz, S. (2016). Style that's sustainable: A new fast-fashion formula. McKinsey \& Company. Retrieved from http://www.mckinsey.com/businessfunctions/sustainability-and-resource-productivity/our-insights/style-thats-sustainable-anew-fast-fashion-formula?cid=sustainability-eml-alt-mip-mck-oth-1610

Richardson, P. S., Dick, A. S., \& Jain, A. K. (1994). Extrinsic and intrinsic cue effects on perceptions of store brand quality. Journal of Marketing, 58(4), 28-36. 
Rickman, T. A., \& Cosenza, R. M. (2007). The changing digital dynamics of multichannel marketing: The feasibility of the weblog: text mining approach for fast fashion trending. Journal of Fashion Marketing and Management, 11(4), 604-621.

Robertson, T. S. (1976). Low-commitment consumer behavior. Journal of Advertising Research, 16(April), 19-24.

Robinson, D. E. (1958). Fashion theory and product design. Harvard Business Review, 36(6), $126-138$.

Robinson, D. E. (1961). The economics of fashion demand. The Quarterly Journal of Economics, 376-398.

Robinson, T. E., \& Berridge, K. C. (1993). The neural basis of drug craving: an incentivesensitization theory of addiction. Brain Research Reviews, 18(3), 247-291.

Rocamora, A. (2002). Fields of Fashion Critical insights into Bourdieu's sociology of culture. Journal of Consumer Culture, 2(3), 341-362.

Rocamora, A. (2011). Personal fashion blogs: Screens and mirrors in digital self-portraits. Fashion Theory, 15(4), 407-424.

Rocamora, A. (2017). Mediatization and Digital Media in the Field of Fashion. Fashion Theory Journal of Dress Body and Culture, 21(5), 505-522. https://doi.org/10.1080/1362704X.2016.1173349

Roos, S., Zamani, B., Sandin, G., Peters, G. M., \& Svanström, M. (2016). A life cycle assessment (LCA)-based approach to guiding an industry sector towards sustainability: The case of the Swedish apparel sector. Journal of Cleaner Production, 133, 691-700.

Rudd, N. A., \& Lennon, S. (2001). Body image: Linking aesthetics and social psychology of appearance. Clothing and Textiles Research Journal, 19(3), 120-133.

Scaraboto, D., \& Fischer, E. (2012). Frustrated fatshionistas: An institutional theory perspective on consumer quests for greater choice in mainstream markets. Journal of Consumer Research, 39(6), 1234-1257.

Schaefer, M., \& Rotte, M. (2007). Favorite brands as cultural objects modulate reward circuit. Neuroreport, 18(2), 141-145.

Schaltegger, S., \& Wagner, M. (2011). Sustainable entrepreneurship and sustainability 
innovation: categories and interactions. Business Strategy and the Environment, 20(4), 222237.

Scheffer, M. (2013). Fashion and business: Problems and solutions. In J. Brand \& J. Teunissen (Eds.), Fashion odyssey: Progress in fashion and sustainability. Arnhem, Netherlands: ArtEZ Press.

Seo, M. G., \& Creed, W. D. (2002). Institutional contradictions, praxis, and institutional change: A dialectical perspective. Academy of Management Review, 27(2), 222-247.

Seuring, S., \& Müller, M. (2008). From a literature review to a conceptual framework for sustainable supply chain management. Journal of Cleaner Production, 16(15), 1699-1710.

Simon, H. A. (1996). The sciences of the artificial (3rd ed.). Cambridge, MA: MIT press.

Smith, A. (2012). Wealth of nations. Hertfordshire, UK: Wordsworth Editions Limited.

Smith, P., \& Riley, A. (2011). Cultural theory: An introduction. Oxford, UK: Blackwell.

Sproles, G. B. (1974). Fashion Theory: A Conceptual Framework. Advances in Consumer Research, 1, 463-472.

Štefko, R., \& Steffek, V. (2018). Key Issues in slow fashion: current challenges and future perspectives. Sustainability, 10(7), 2270.

Stoecker, R. (1991). Evaluating and rethinking the case study. The Sociological Review, 39(1), $88-112$.

Stubbs, W., \& Cocklin, C. (2008). Conceptualizing a "sustainability business model." Organization \& Environment, 21(2), 103-127.

Sustainable Apparel Coalition. (2017). The Higg Index. Retrieved from http://apparelcoalition.org/the-higg-index/

Sustainable Apparel Coalition. (2018). Higg Materials Sustainability Index. Retrieved from https://msi.higg.org/page/msi-home

Sustainable Apparel Coalition. (2019). Our members. Retrieved from https://apparelcoalition.org/brands-retailers/

Tam, L., Wood, W., \& Song, M. J. (2009). Brand loyalty is not habitual. In D. J. MacInnis, C. W. Park, \& J. R. Priester (Eds.), Handbook of brand relationships (pp. 43-62). Armonk, 
NY: M.E. Sharpe.

Teunissen, J. (2013). Fashion Odyssey. In J. Brand \& J. Teunissen (Eds.), Fashion odyssey: Progress in fashion and sustainability. Arnhem, Netherlands: ArtEZ Press.

Textile Environment Design. (2006). The Ten. Retrieved from http://www.tedresearch.net/tedsten/

Textile Environment Design. (2018). Ted's Ten. Retrieved from http:/www.tedresearch.net/tedsten-aims/

Thorisdottir, T. S., \& Johannsdottir, L. (2019). Sustainability within fashion business models: A systematic literature review. Sustainability, 11(8), 2233.

https://doi.org/http://doi:10.3390/su11082233

Tongco, M. D. (2007). Purposive sampling as a tool for informant selection. Ethnobotany Research \& Applications, 5, 147-158.

Troy, N. (2003). Couture culture: A study of modern art and fashion. Cambridge, MA: MIT Press.

Tseng, M.-L., Tan, R. R., \& Siriban-Manalang, A. B. (2013). Sustainable consumption and production for Asia: sustainability through green design and practice. Journal of Cleaner Production, 40, 1-5.

Tukker, Arnold. (2015). Product services for a resource-efficient and circular economy-a review. Journal of Cleaner Production, 97, 76-91.

United Nations. (2019). Micro-, Small and Medium-sized Enterprises Day. Retrieved April 28, 2019, from https://www.un.org/en/events/smallbusinessday/

van der Velden, N. M., Patel, M. K., \& Vogtländer, J. G. (2014). LCA benchmarking study on textiles made of cotton, polyester, nylon, acryl, or elastane. The International Journal of Life Cycle Assessment, 19(2), 331-356.

Veryzer, R. W. (2000). Design and consumer research. Design Management Journal, 1(2), 6473.

Vezzoli, C., \& Manzini, E. (2009). Review: Design for sustainable production consumption and production systems. In A Tukker, M. Charter, C. Vezzoli, E. Sto, \& M. Andersen (Eds.), System Innovation for Sustainability (pp. 138-158). Seffield, UK: Greenleaf . 
von Hippel, E. A. (2017). Free Innovation. Cambridge, MA: MIT Press.

Wacquant, L. (2005). Habitus. In J. Becket \& Z. Milan (Eds.), International encyclopedia of economic sociology. London: Routledge.

Walker, S. (2006). Sustainable by design: Explorations in theory and practice. London, UK: Earthscan.

Walter, M. (2009). Participatory action research. Social research methods. Oxford, UK: Oxford University .

Wang, G. (2002). Attitudinal correlates of brand commitment: An empirical study. Journal of Relationship Marketing, 1(2), 57-75.

Watkins, S. M. (1988). Using the design process to teach functional apparel design. Clothing and Textiles Research Journal, 7(1), 10-14.

WCED, \& Press, O. U. (1987). Our Common Future. Oxford, UK: World Commission on Environment and Development.

Webster, K. (2016). Fusion of artisan and virtual: Fashion's new world opportunities. In A. Vecchi \& C. Buckley (Eds.), Handbook of research on global fashion management and merchandising (pp. 346-372). Hershey, PA: IGI Global.

Wilkinson, L., \& Friendly, M. (2009). The history of the cluster heat map. The American Statistician, 63(2), 179-184.

Williams, D. (2013). Fashion Education in Sustainability: Change Through Experiential Crossings. Ualresearchonline.Arts.Ac.Uk. Retrieved from http://ualresearchonline.arts.ac.uk/6305/1/TESSNET_Dilys_Williams__Fashion_Education_in_Sustainability.pdf

Wilson, E. (2003). Adorned in dreams: Fashion and modernity. IB Tauris.

Wimmer, W., Züst, R., \& Lee, K.-M. (2004). Ecodesign Implementation. Dordrecht: Springer.

Winkler, H. (2011). Closed-loop production systems-A sustainable supply chain approach. CIRP Journal of Manufacturing Science and Technology, 4(3), 243-246. https://doi.org/10.1016/j.cirpj.2011.05.001

Winter, S., \& Lasch, R. (2016). Environmental and social criteria in supplier evaluation-Lessons from the fashion and apparel industry. Journal of Cleaner Production, 139, 175-190. 
Wölfel, C., \& Merritt, T. (2013). Method card design dimensions: A survey of card-based design tools. In P. Kotźe (Ed.), INTERACT (Vol. 8117 LNCS, pp. 479-486).

https://doi.org/10.1007/978-3-642-40483-2_34

Wood, W., \& Neal, D. T. (2009). The habitual consumer. Journal of Consumer Psychology, 19(4), 579-592.

Wood, W., Quinn, J. M., \& Kashy, D. A. (2002). Habits in everyday life: thought, emotion, and action. Journal of Personality and Social Psychology, 83(6), 1281.

Wood, W., Tam, L., \& Witt, M. G. (2005). Changing circumstances, disrupting habits. Journal of Personality and Social Psychology, 88(6), 918-933.

Woodward, S. (2009). The myth of street style. Fashion Theory: The Journal of Dress, Body \& Culture, 13(1), 83-102.

Yelavich, S., \& Adams, B. (Eds.). (2014). Design as Future-making. New York, NY:

Bloomsbury Publishing.

Zafarmand, S. J., Sugiyama, K., \& Watanabe, M. (2003). Aesthetic and Sustainability: The Aesthetic Attributes Promoting Product Sustainability. The Journal of Sustainable Product Design, 3(3-4), 173-186. https://doi.org/10.1007/s10970-005-6157-0 